\title{
Automated Glycan Assembly of Complex Oligosaccharides Related to Blood Group Determinants
}

\author{
Heung Sik Hahm, ${ }^{[a, b]}$, Chien-Fu Liang, ${ }^{[a, c]}$ Chian-Hui Lai, ${ }^{[a, d]}$ Richard J. Fair, ${ }^{[a]}$ Frank \\ Schuhmacher ${ }^{[a, b]}$ and Peter H. Seeberger* ${ }^{[a, b]}$ \\ ${ }^{a}$ Max Planck Institute of Colloids and Interfaces, Department of Biomolecular System, Am Mühlenberg 1, 14424, \\ Potsdam, Germany \\ ${ }^{\mathrm{b}}$ Freie Universität Berlin, Institute of Chemistry and Biochemistry, Arnimallee 22, 14195, Berlin, Germany \\ ${ }^{\mathrm{c}}$ Department of Chemistry, National Chung Hsing University, 145 Xingda Rd., South Dist., Taichung City 402 , \\ Taiwan. \\ ${ }^{\mathrm{d}}$ Genomic Research Center, Academia Sinica, 128, Section 2, Academia Road, Taipei 115, Taiwan.
}

Fax: (+49) 30838459301

E-mail: peter.seeberger@mpikg.mpg.de

NMR spectra for monomer building blocks $\mathrm{S} 2-24$

Table S1 for automated reaction conditions S24

Analytical NP-HPLC chromatogram for the linker stabililty study (Figure S1) S25

NMR spectra and Analytical NP-HPLC chromatogram for protected oligosaccharides ... S26-53

NMR spectra for the conjugation-ready unprotected oligosaccharides S54 - 59 


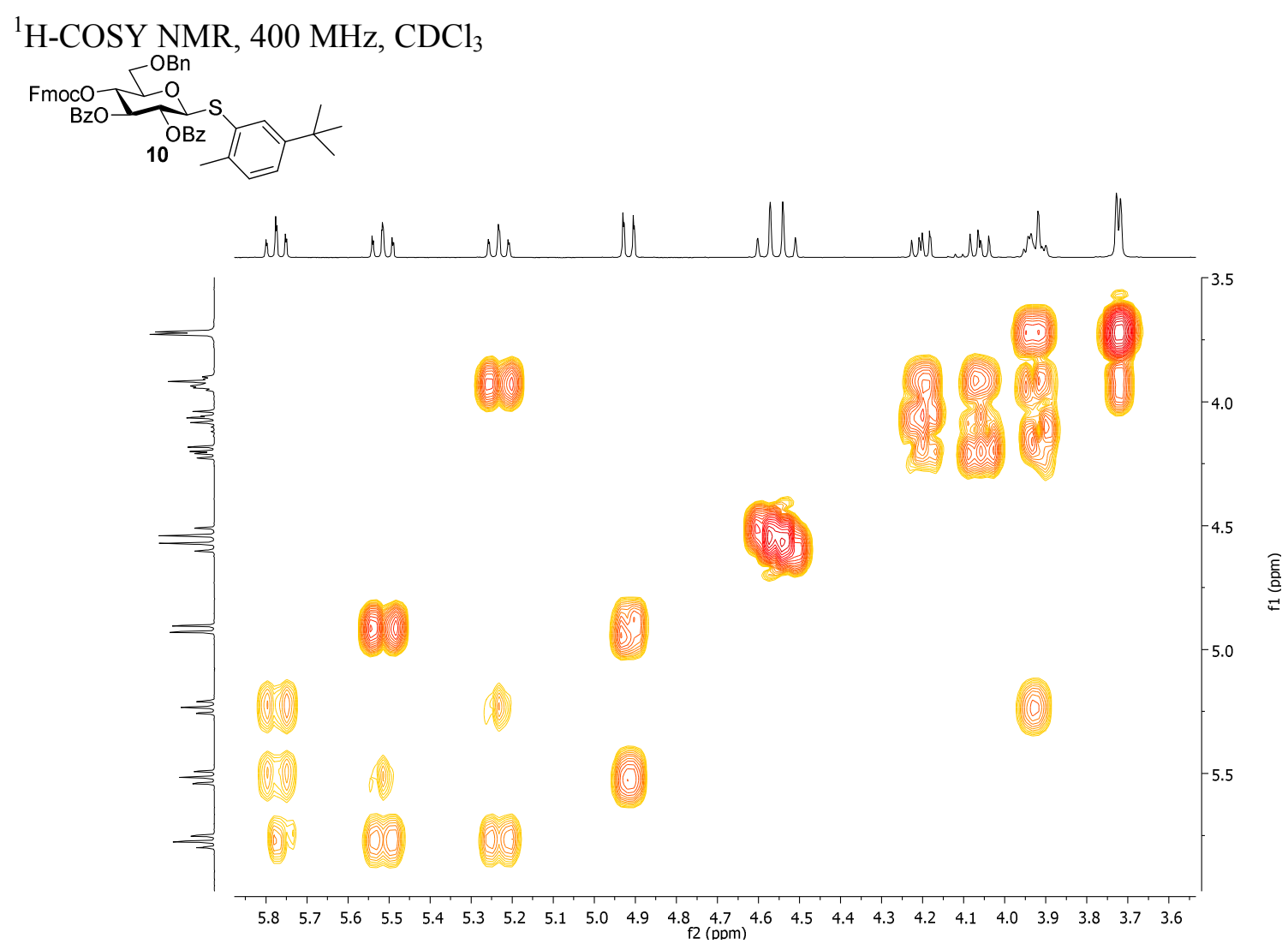

${ }^{1} \mathrm{H}-{ }^{13} \mathrm{C}-\mathrm{HSQC}$ NMR, $400 \mathrm{MHz}, \mathrm{CDCl}_{3}$

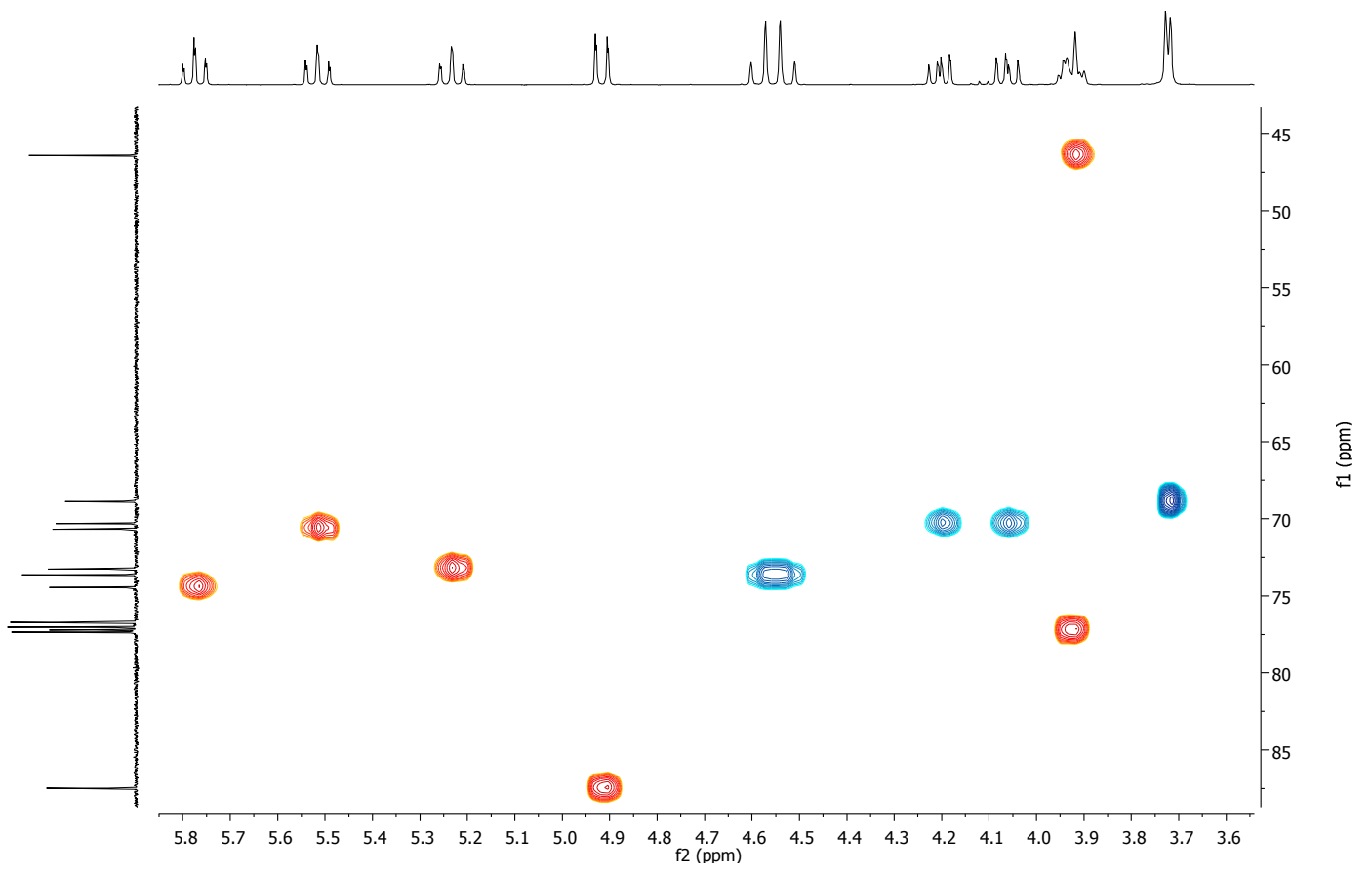


${ }^{1} \mathrm{H}$ NMR, $400 \mathrm{MHz}, \mathrm{CDCl}_{3}$

HHS-CCI-100_PROTON_13Mar12_01

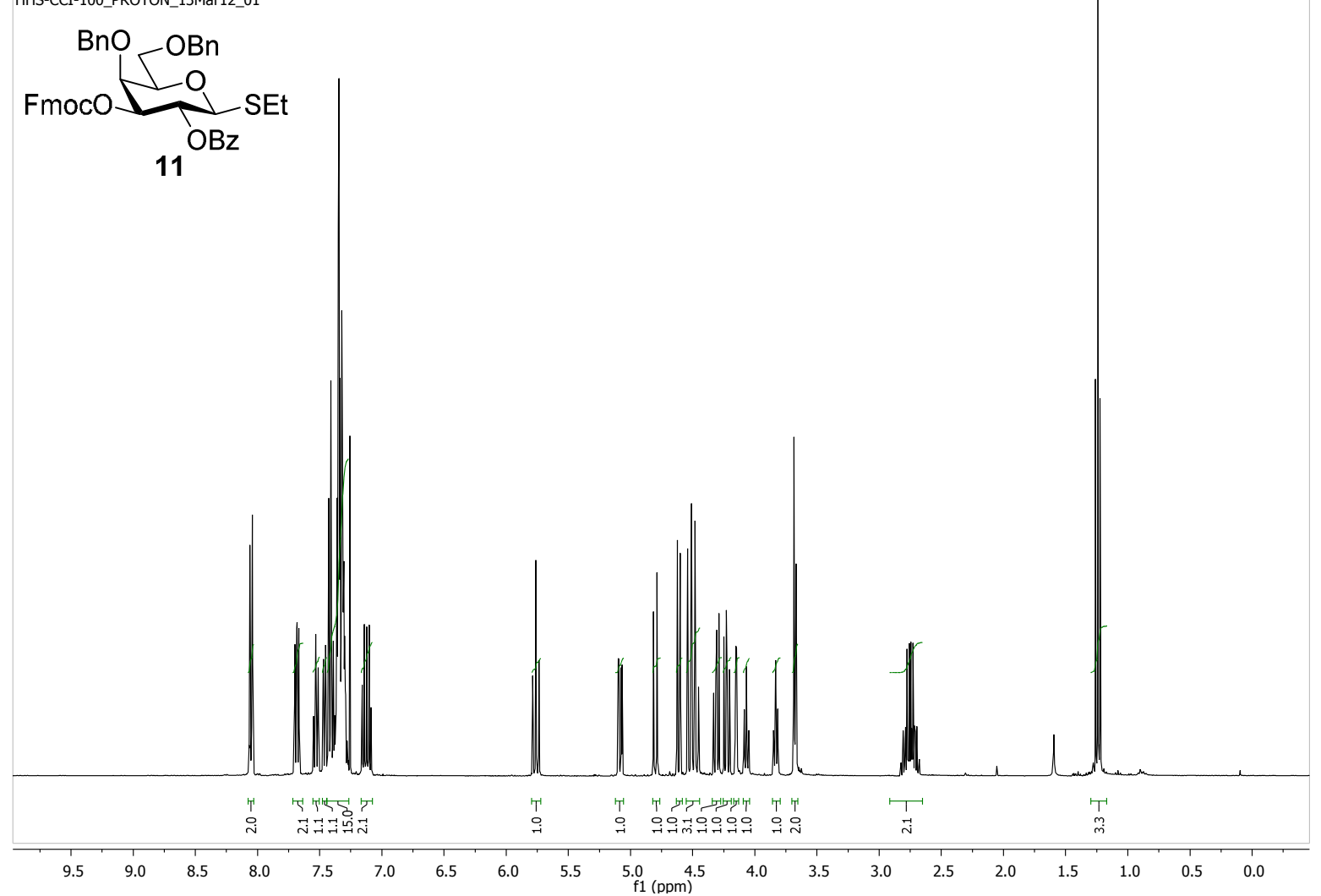

${ }^{13} \mathrm{C} \mathrm{NMR,}, 100 \mathrm{MHz}, \mathrm{CDCl}_{3}$
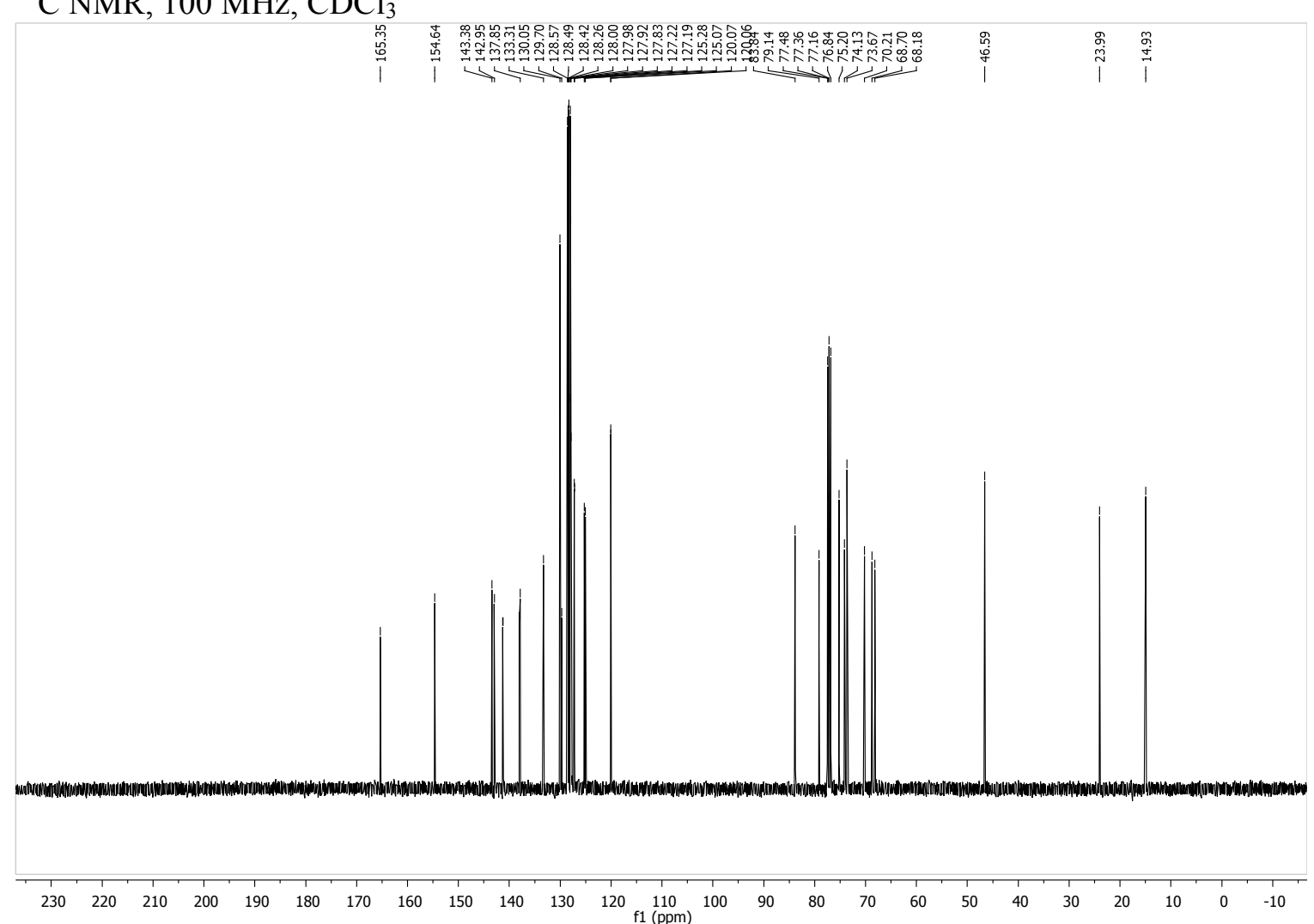
${ }^{1} \mathrm{H}-\mathrm{COSY}$ NMR, $400 \mathrm{MHz}, \mathrm{CDCl}_{3}$
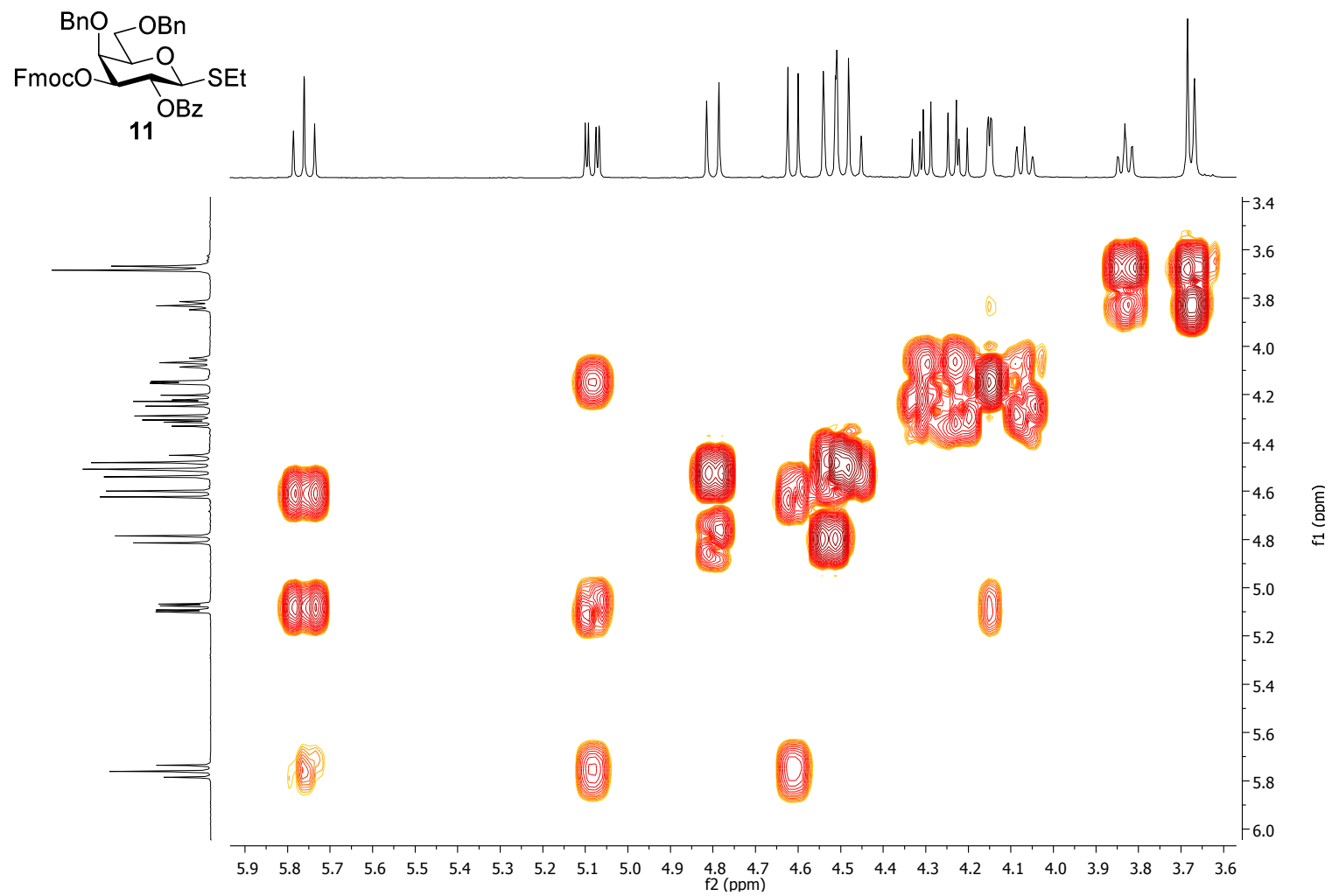

${ }^{1} \mathrm{H}^{-13} \mathrm{C}-\mathrm{HSQC}$ NMR, $400 \mathrm{MHz}, \mathrm{CDCl}_{3}$

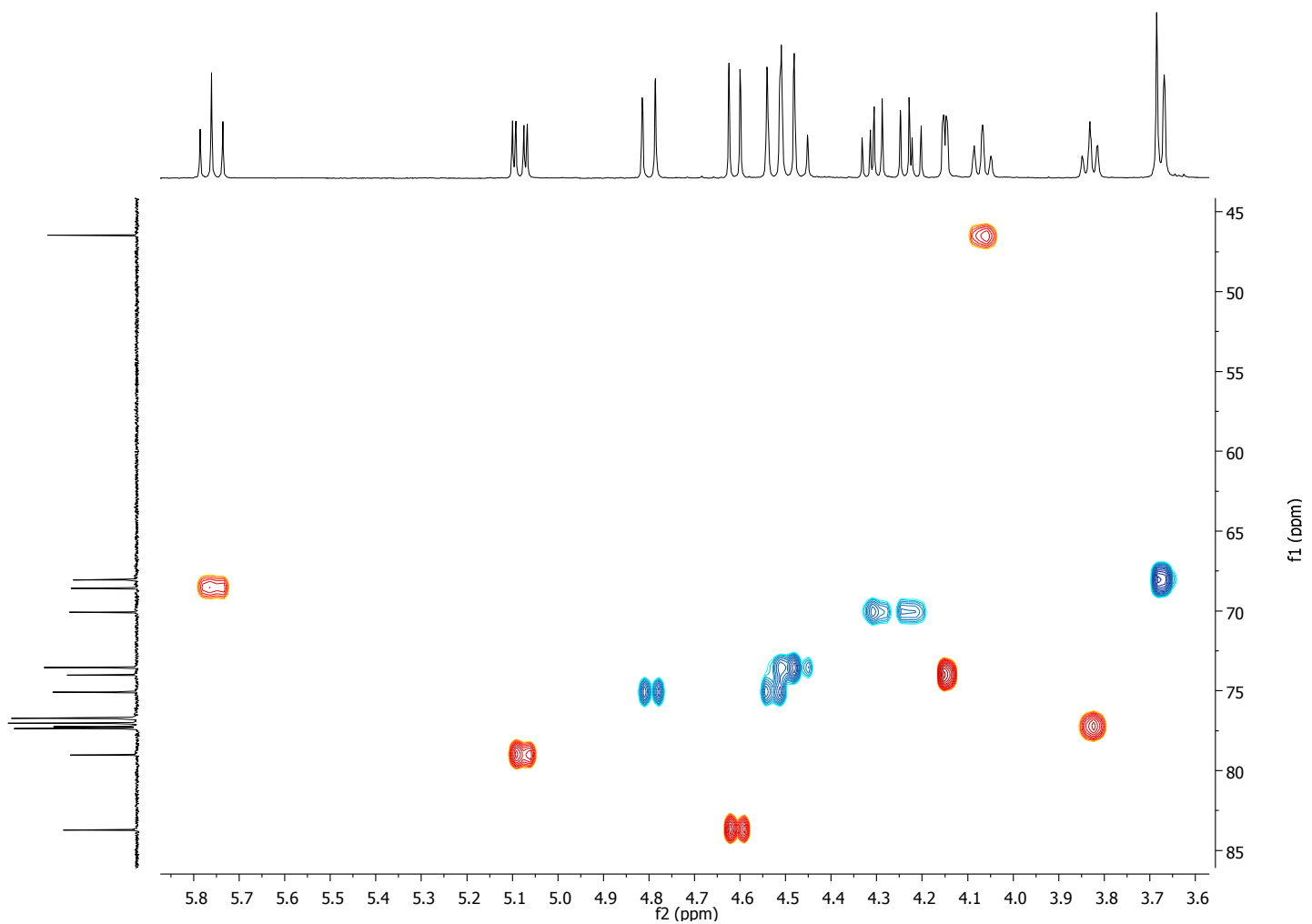


${ }^{1} \mathrm{H}$ NMR, $400 \mathrm{MHz}, \mathrm{CDCl}_{3}$

HHS-CCI-100-4-SP2_PROTON_04Jan13_01

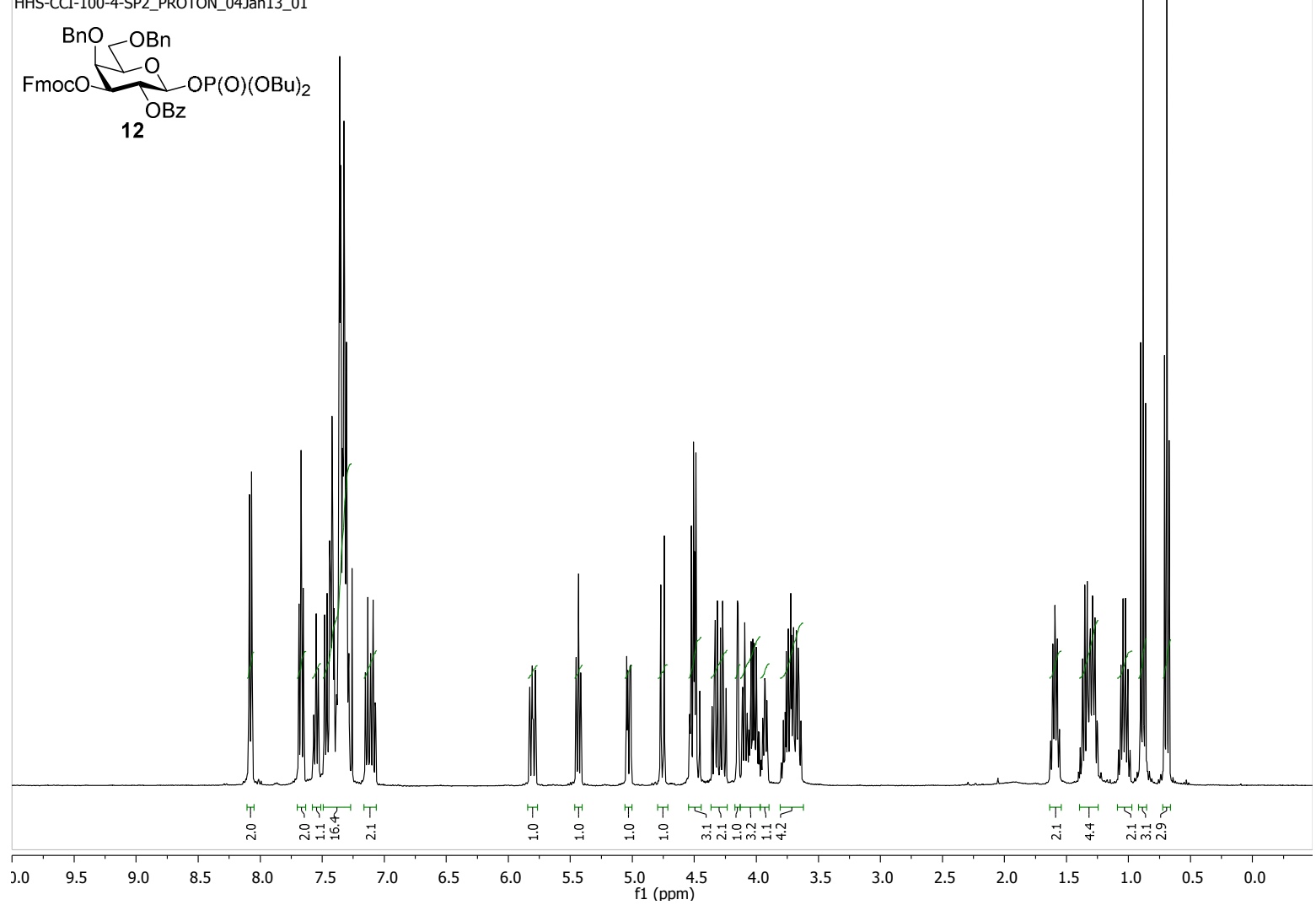

${ }^{13} \mathrm{C} \mathrm{NMR,}, 100 \mathrm{MHz}, \mathrm{CDCl}_{3}$

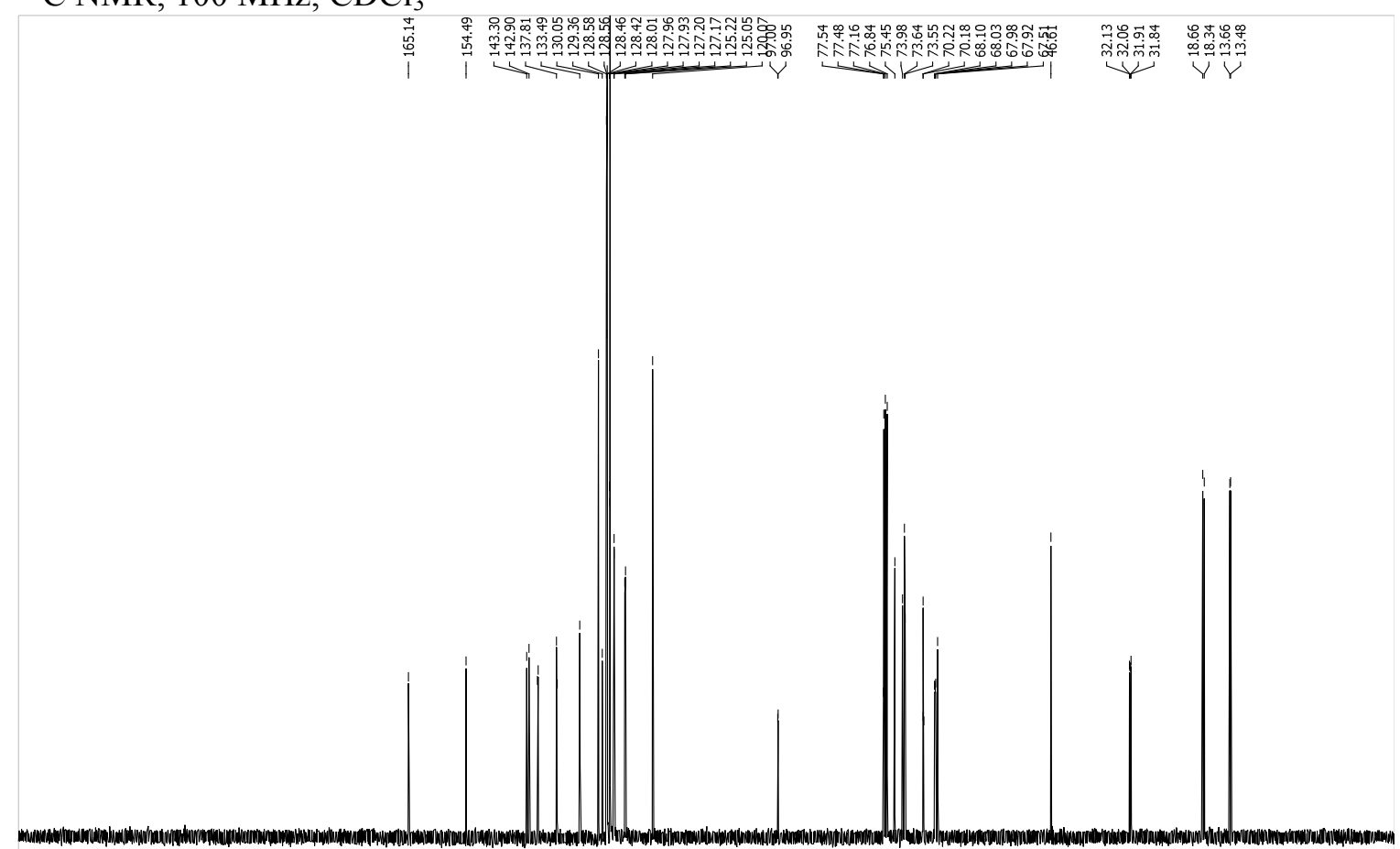

$\begin{array}{llllllllllllllllllllllllllll}230 & 220 & 210 & 200 & 190 & 180 & 170 & 160 & 150 & 140 & 130 & 120 & \underset{f 1}{110} & 100 & 90 & 80 & 70 & 60 & 50 & 40 & 30 & 20 & 10 & 0 & -10\end{array}$ 
${ }^{1} \mathrm{H}-\mathrm{COSY} \mathrm{NMR}, 400 \mathrm{MHz}, \mathrm{CDCl}_{3}$

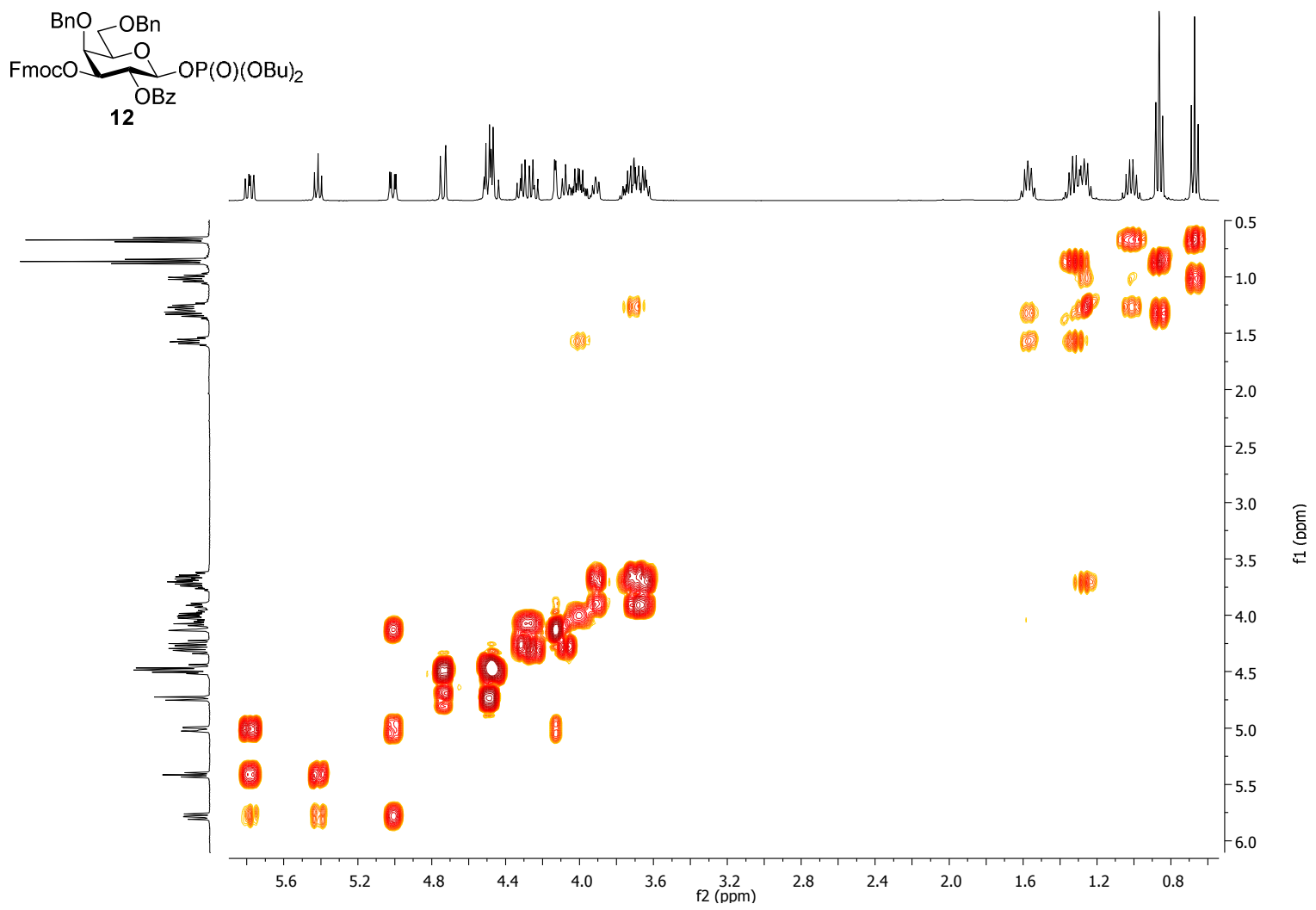

${ }^{1} \mathrm{H}-{ }^{13} \mathrm{C}-\mathrm{HSQC}$ NMR, $400 \mathrm{MHz}, \mathrm{CDCl}_{3}$

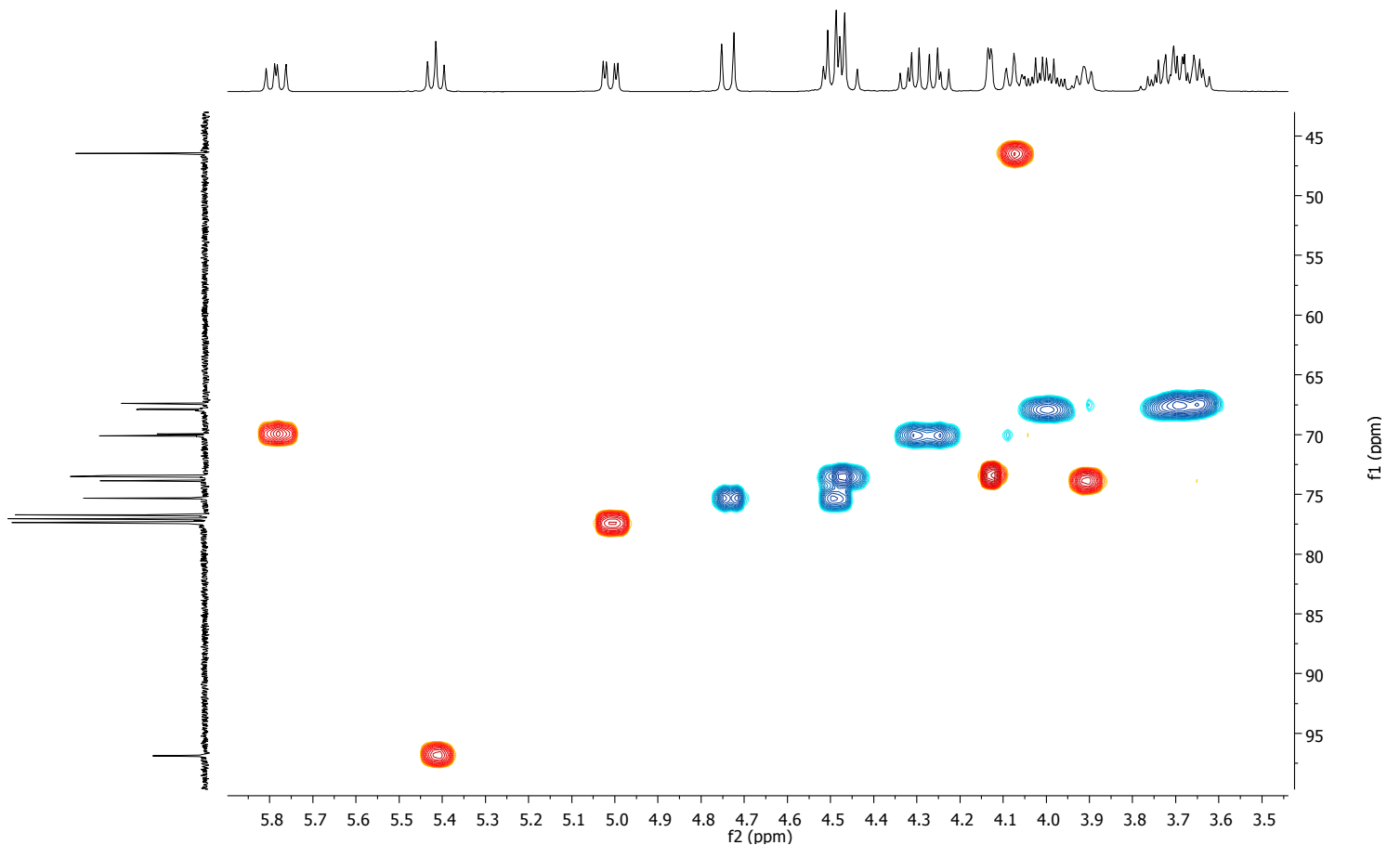


${ }^{31} \mathrm{P}$ NMR, $162 \mathrm{MHz}, \mathrm{CDCl}_{3}$

HHS-CCI-100-4-SP2_PHOSPHORUS_04Jan13_01

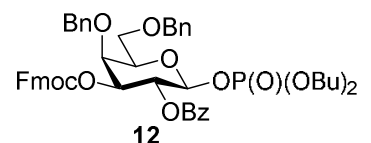

12

${ }^{1} \mathrm{H} \mathrm{NMR}, 400 \mathrm{MHz}, \mathrm{CDCl}_{3}$

HHS-GICNAC-1-030_PROTON_28Mar12_02 HHS-GICNAC-1-030
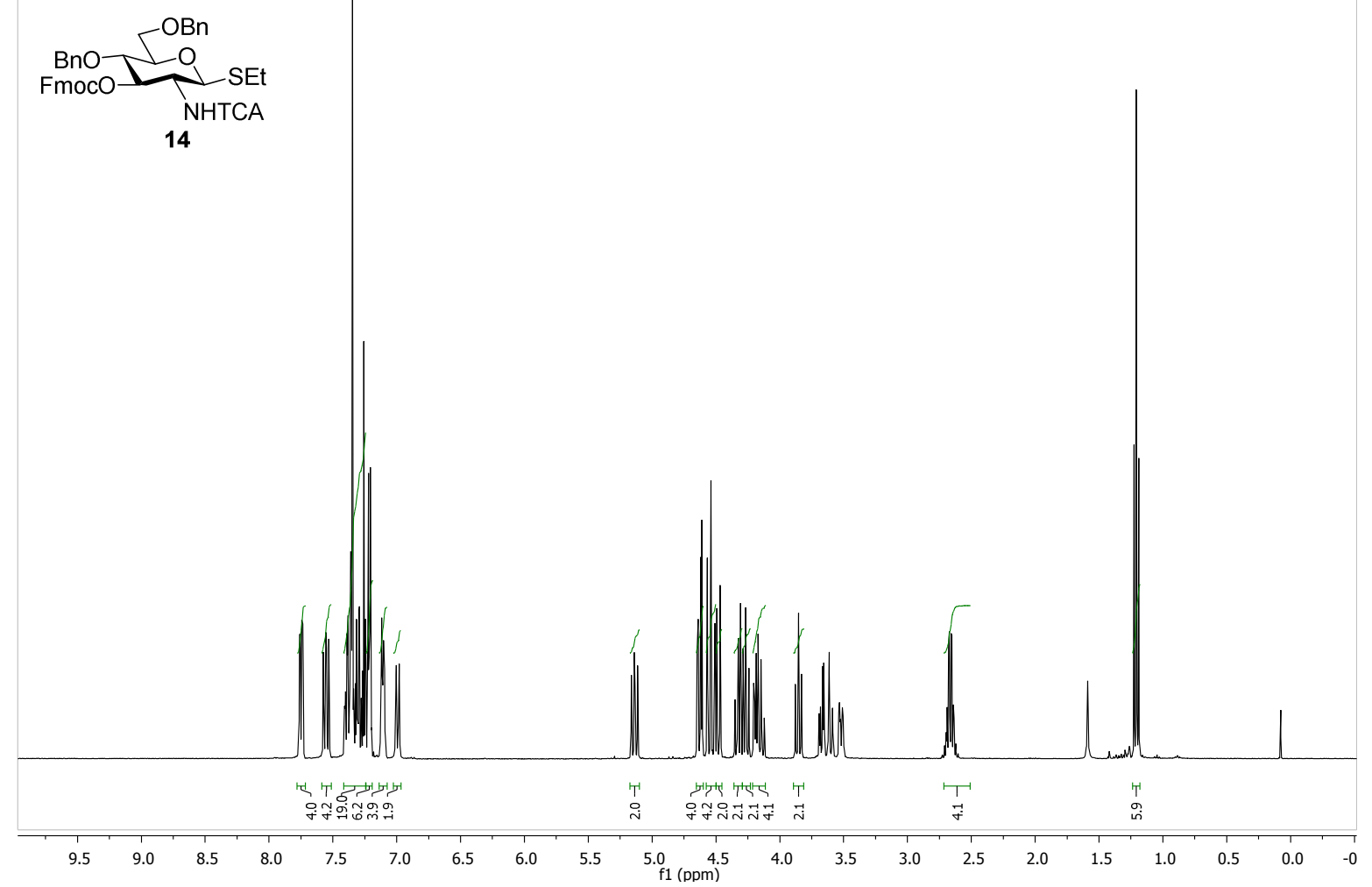

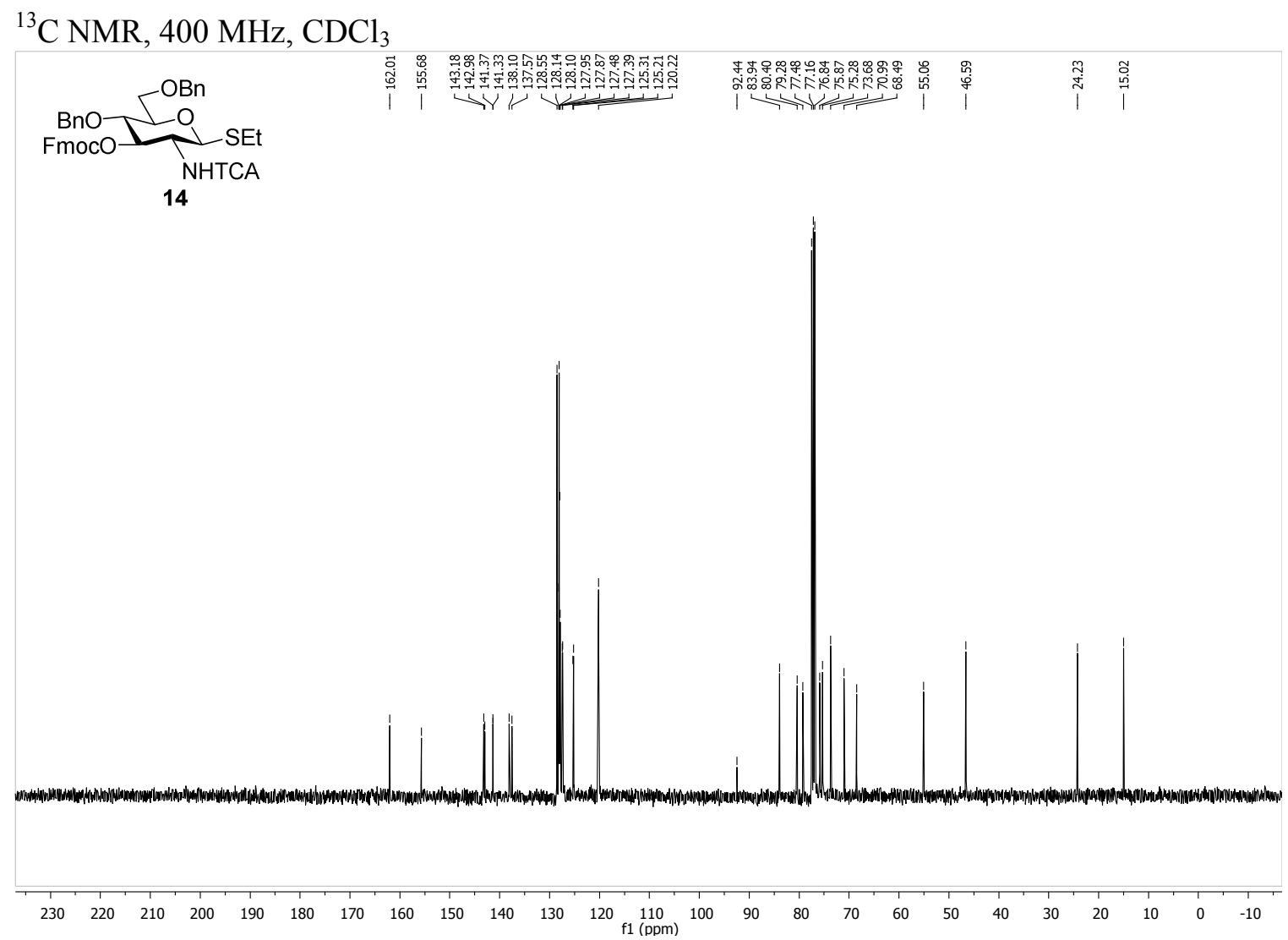

${ }^{1} \mathrm{H}-\mathrm{COSY} \mathrm{NMR}, 400 \mathrm{MHz}, \mathrm{CDCl}_{3}$

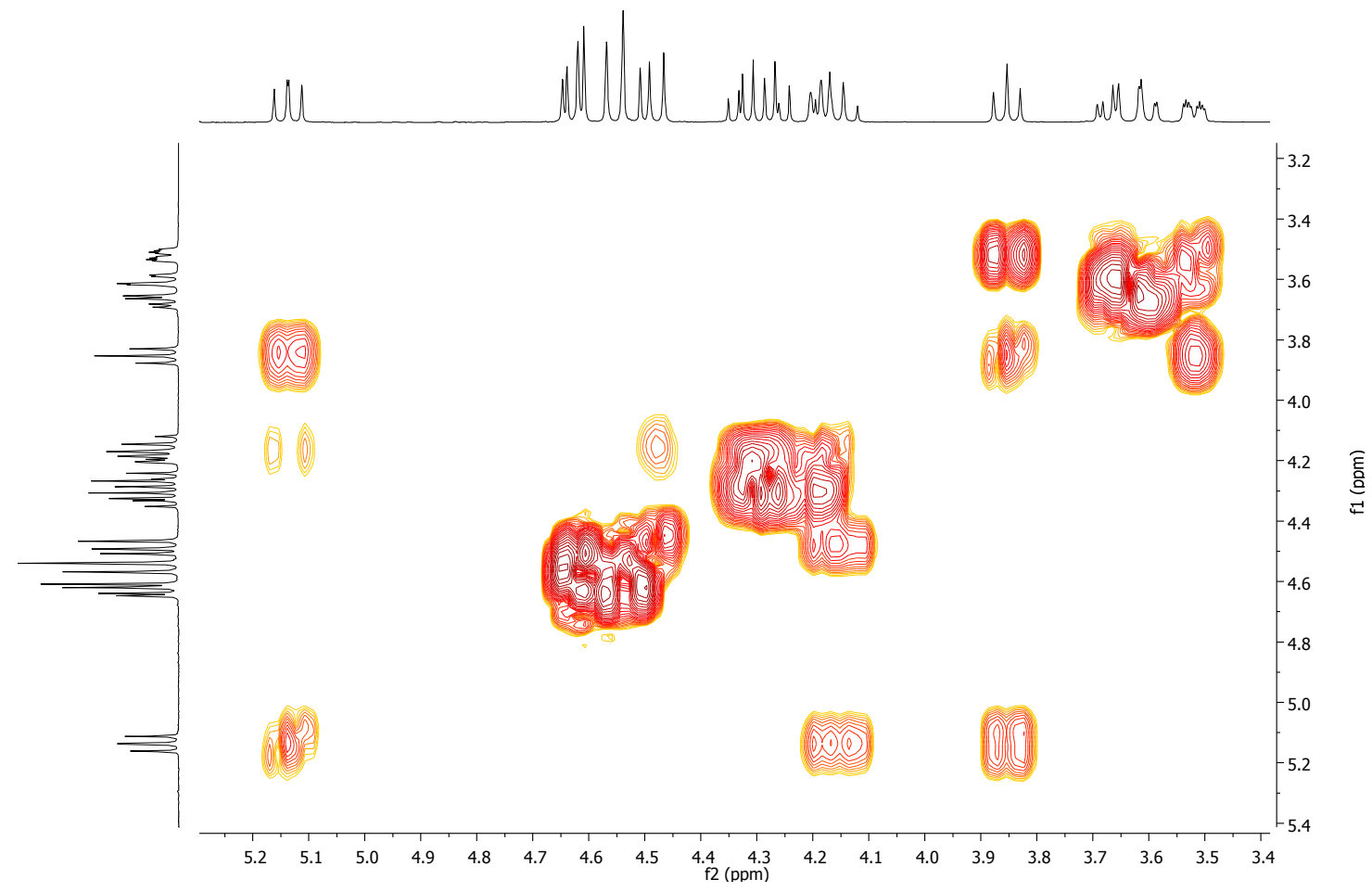


${ }^{1} \mathrm{H}^{13} \mathrm{C}-\mathrm{HSQC}$ NMR, $400 \mathrm{MHz}, \mathrm{CDCl}_{3}$

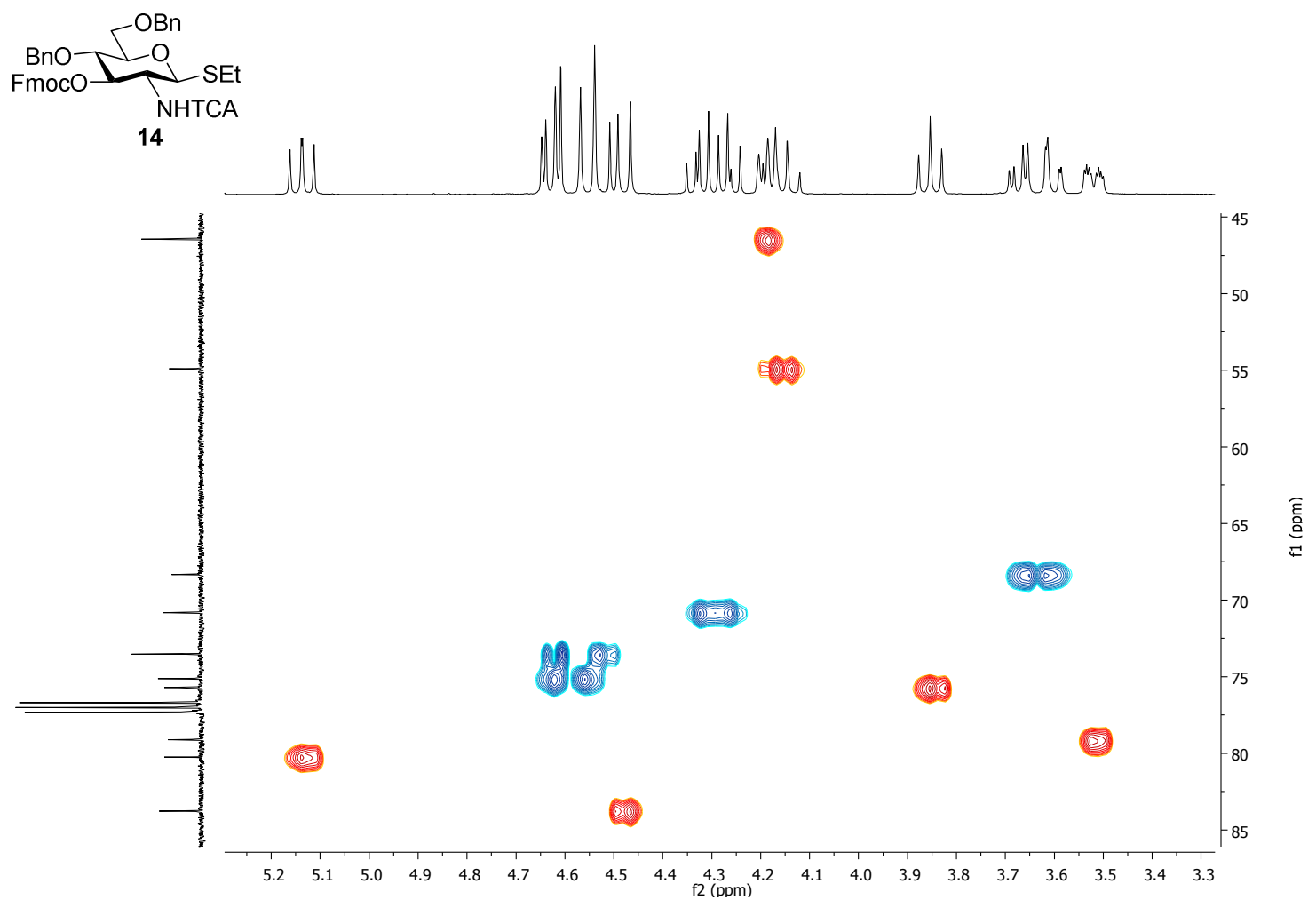

${ }^{1} \mathrm{H}$ NMR, $400 \mathrm{MHz}, \mathrm{CDCl}_{3}$

HHS-GICNAC-1-023_PROTON_14Nov12_01
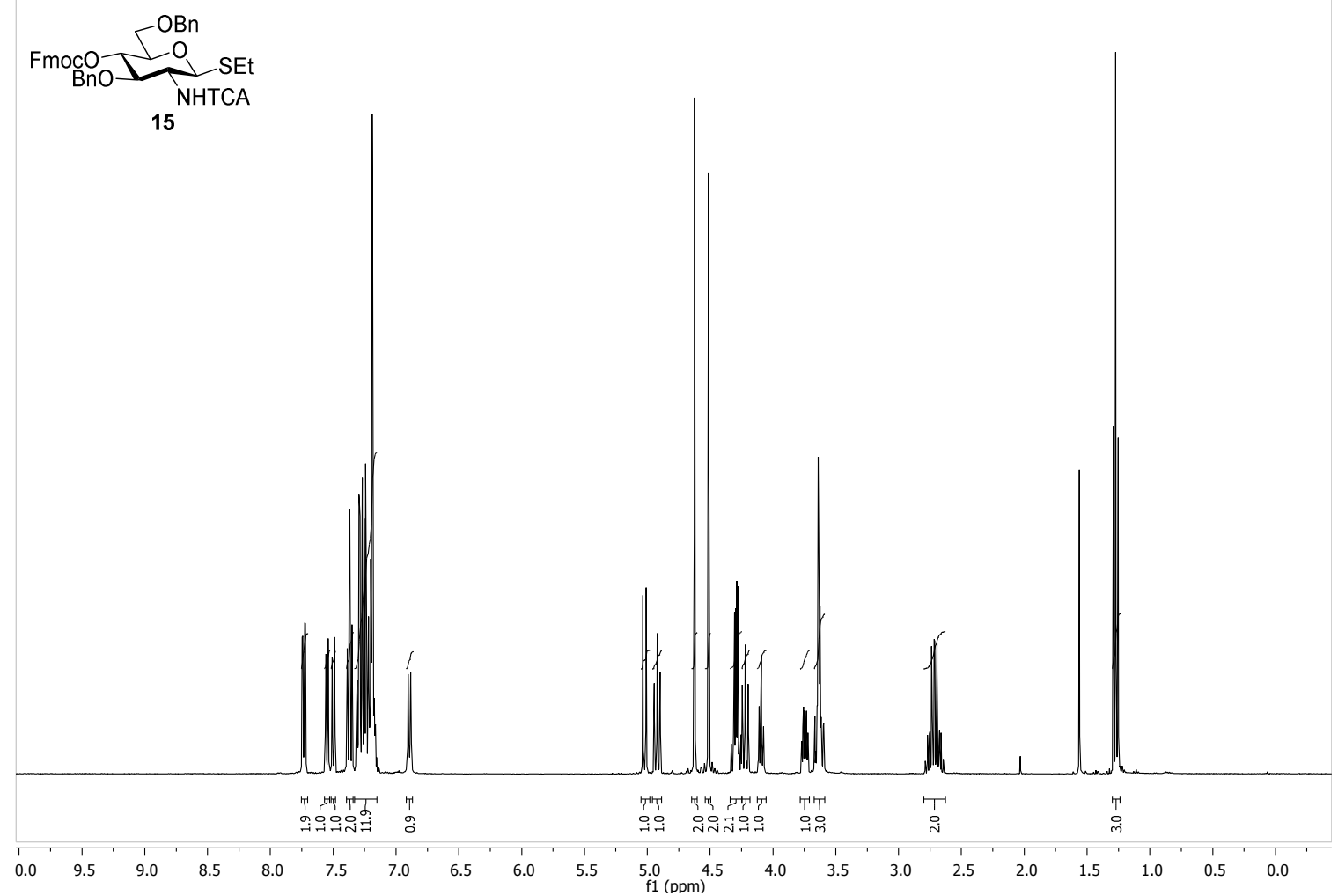

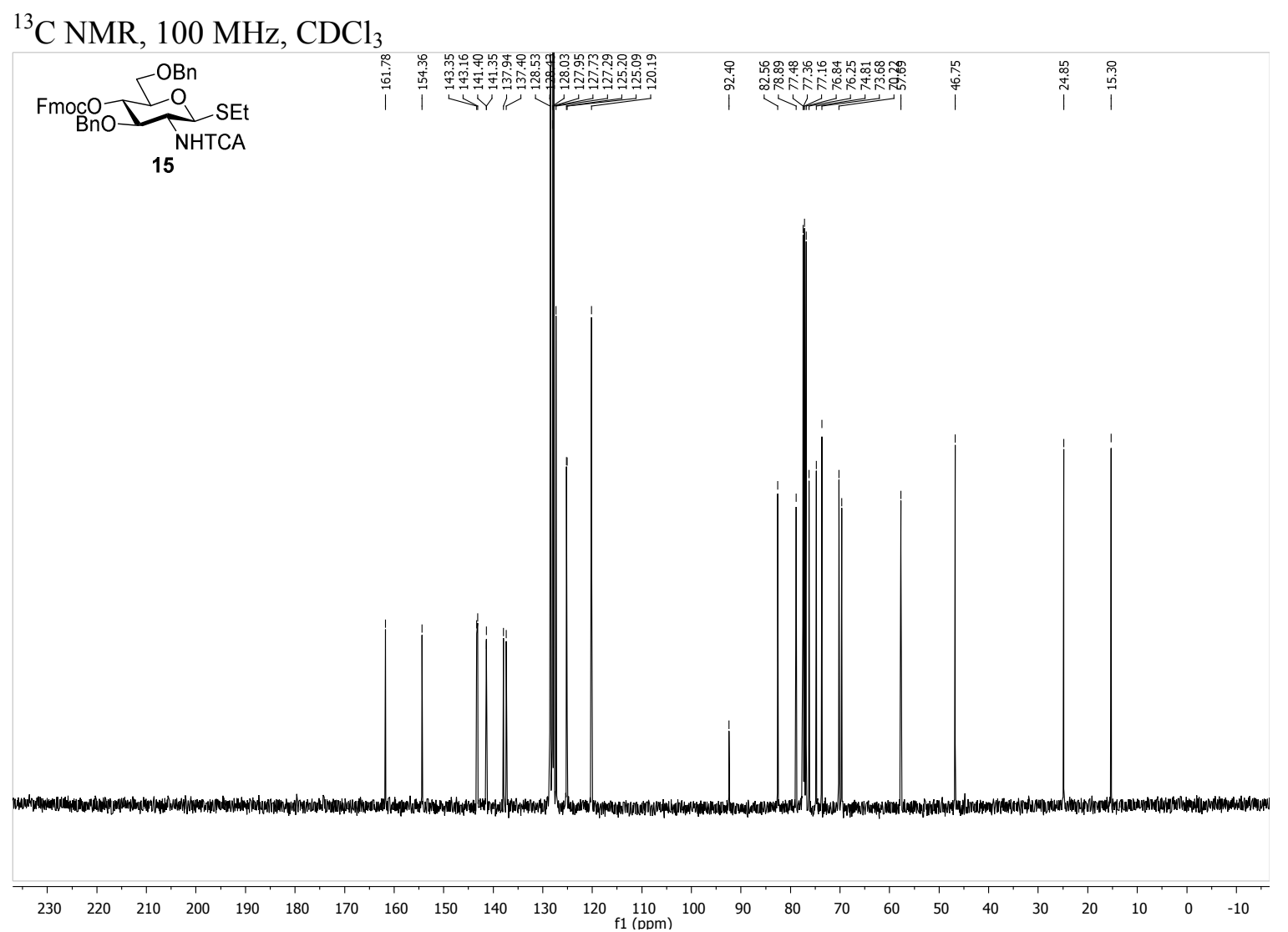

${ }^{1} \mathrm{H}-\mathrm{COSY} \mathrm{NMR}, 400 \mathrm{MHz}, \mathrm{CDCl}_{3}$

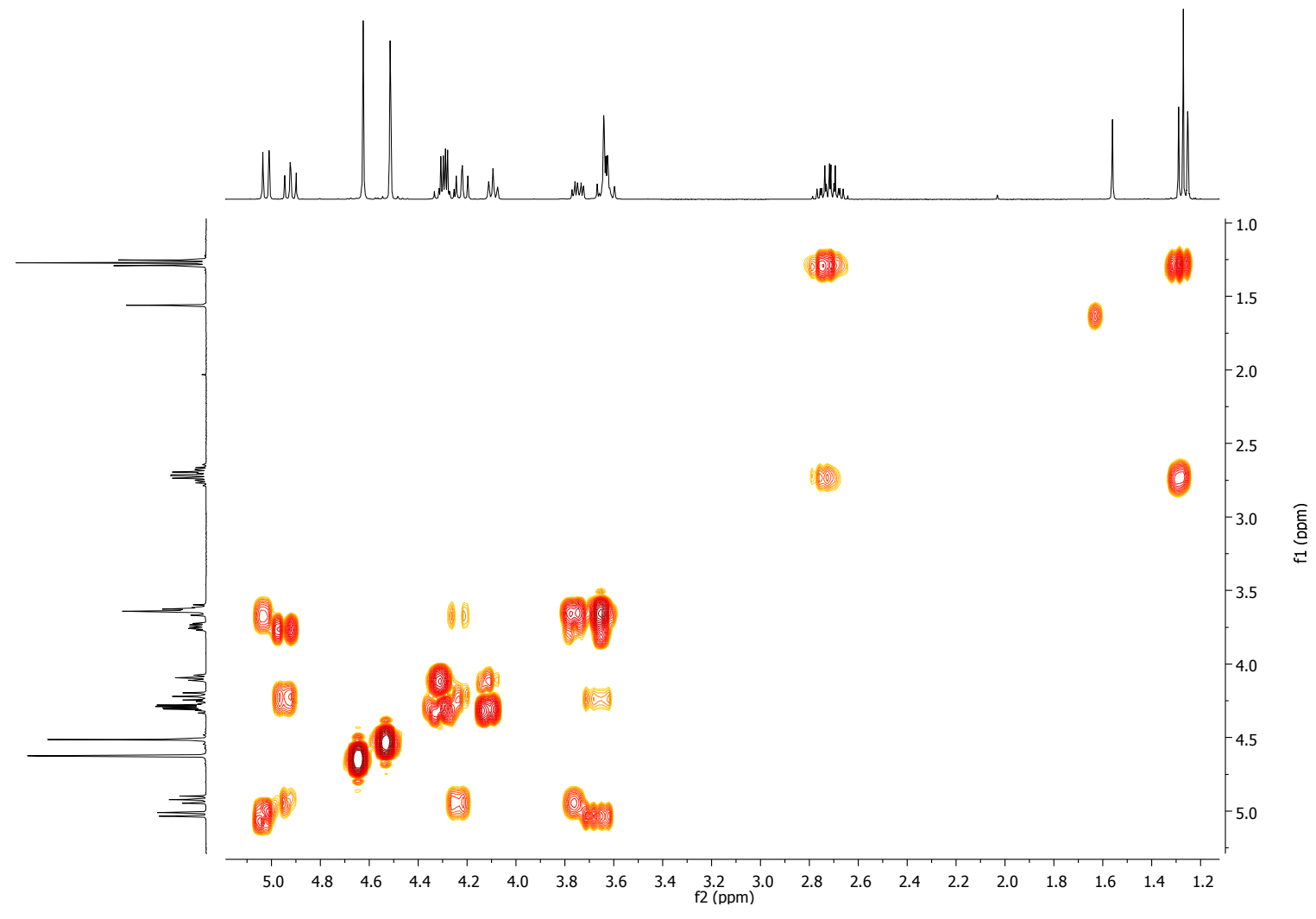


${ }^{1} \mathrm{H}^{13} \mathrm{C}-\mathrm{HSQC} \mathrm{NMR}, 400 \mathrm{MHz}, \mathrm{CDCl}_{3}$

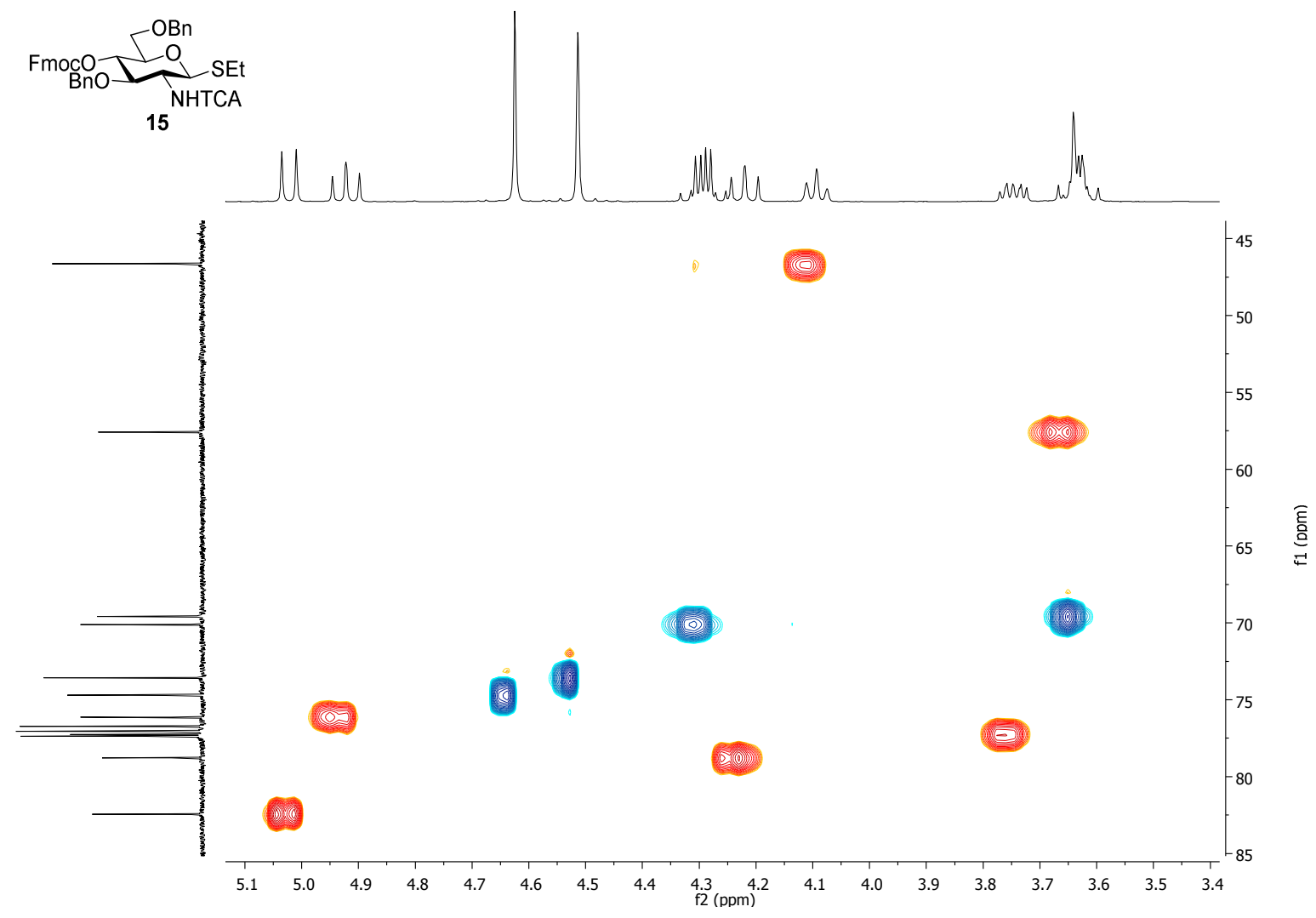

${ }^{1} \mathrm{H}$ NMR, $400 \mathrm{MHz}, \mathrm{CDCl}_{3}$

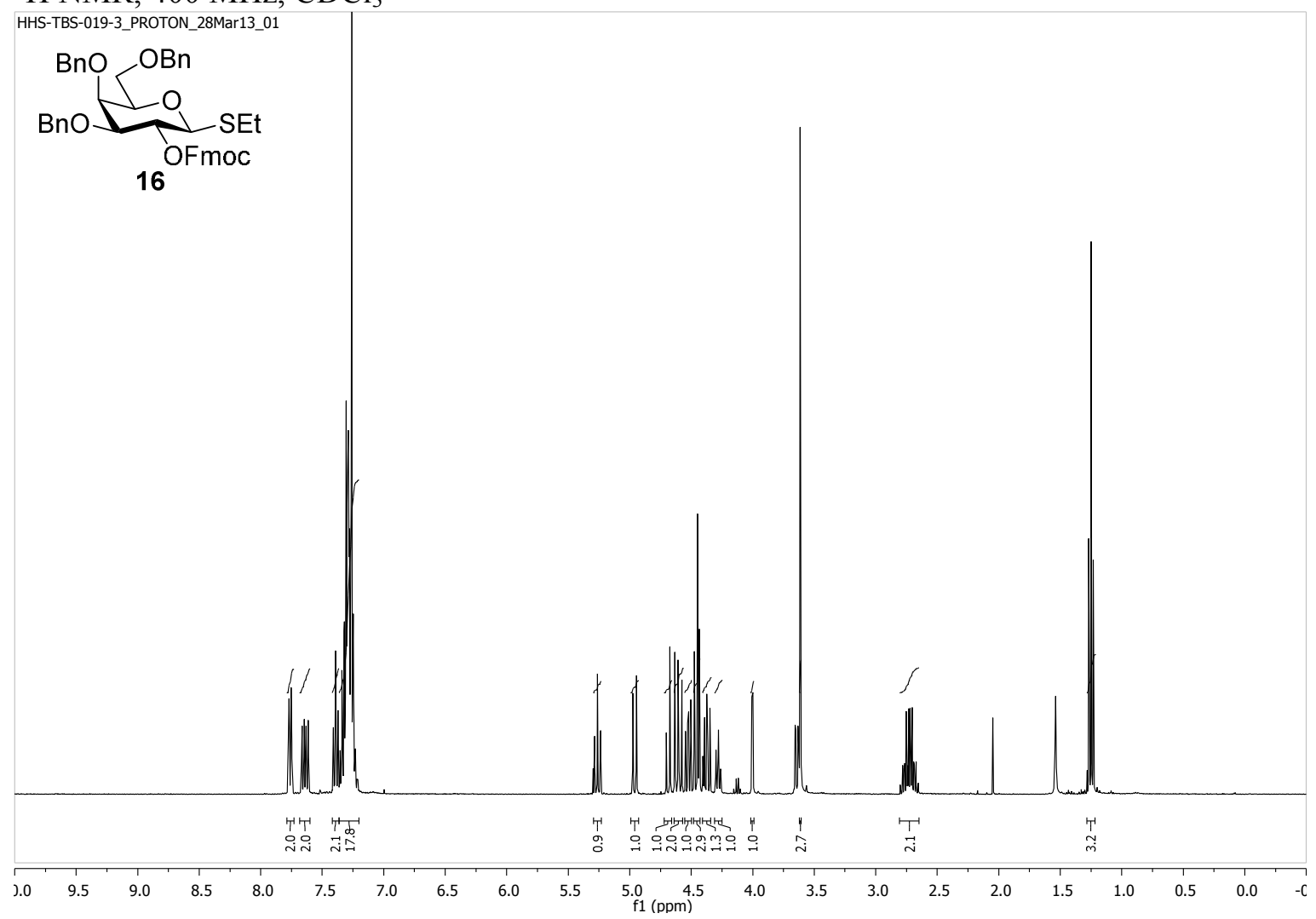



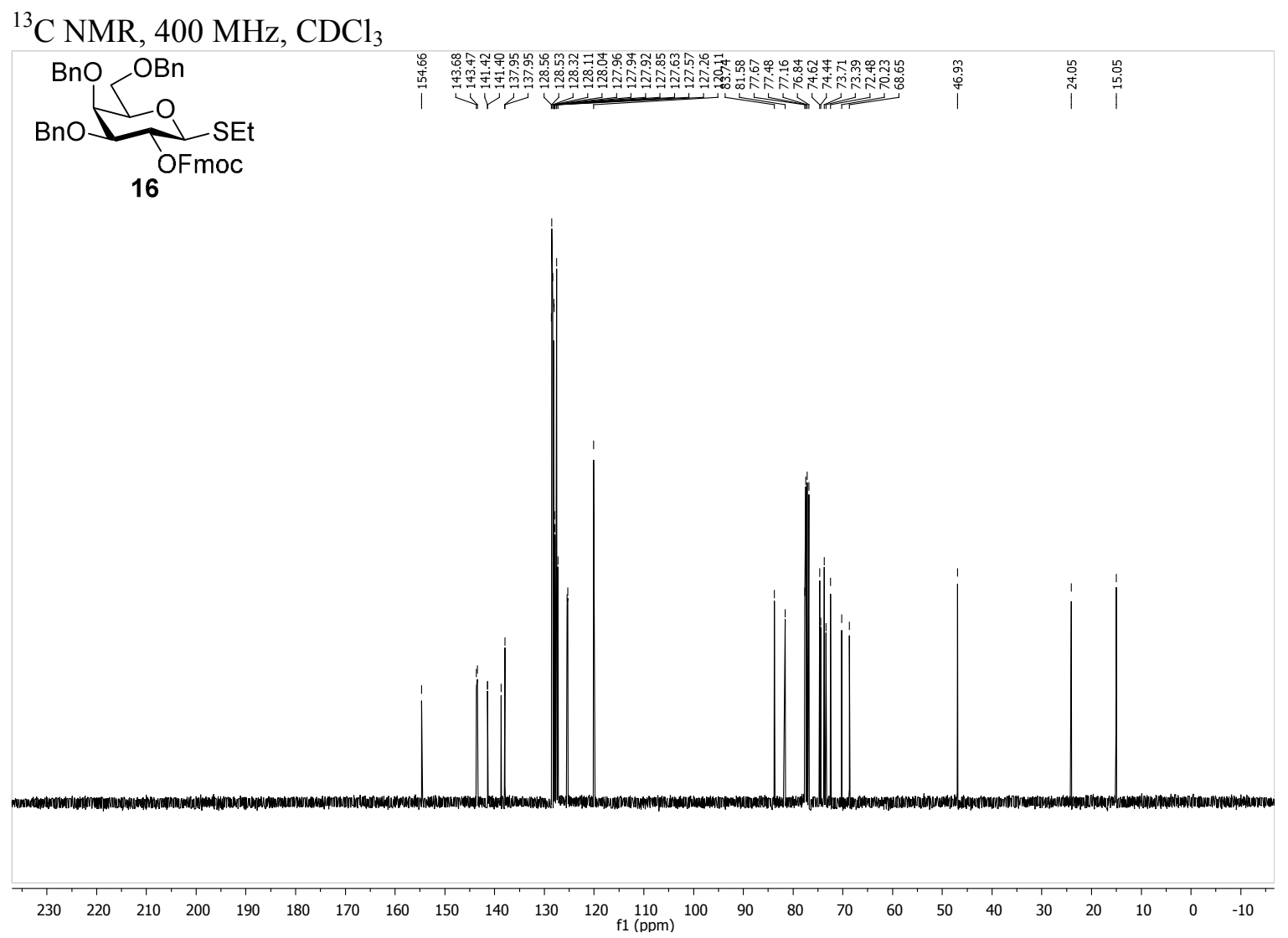

${ }^{1} \mathrm{H}-\mathrm{COSY} \mathrm{NMR}, 400 \mathrm{MHz}, \mathrm{CDCl}_{3}$

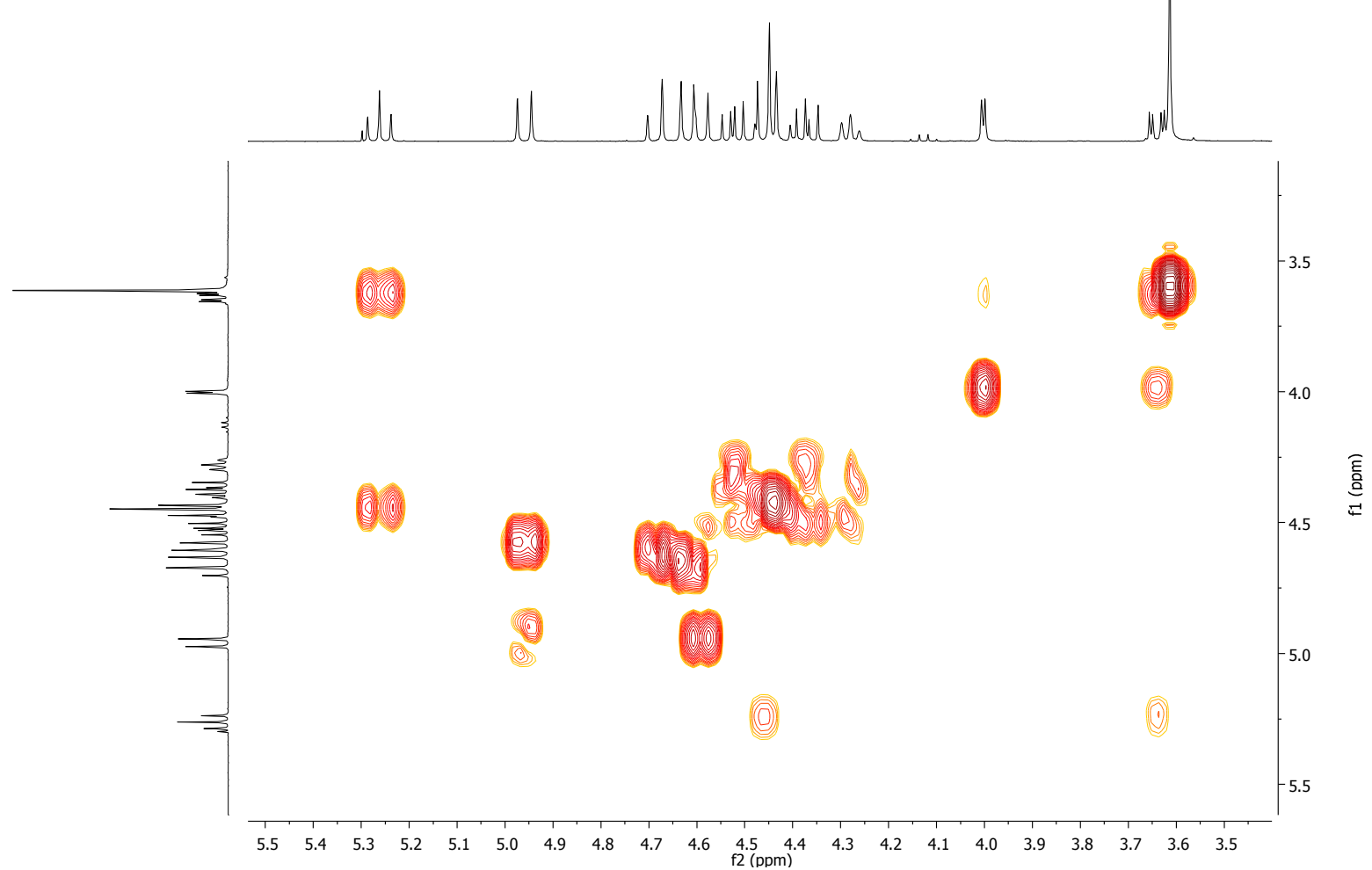


${ }^{1} \mathrm{H}^{13} \mathrm{C}-\mathrm{HSQC} \mathrm{NMR}, 400 \mathrm{MHz}, \mathrm{CDCl}_{3}$

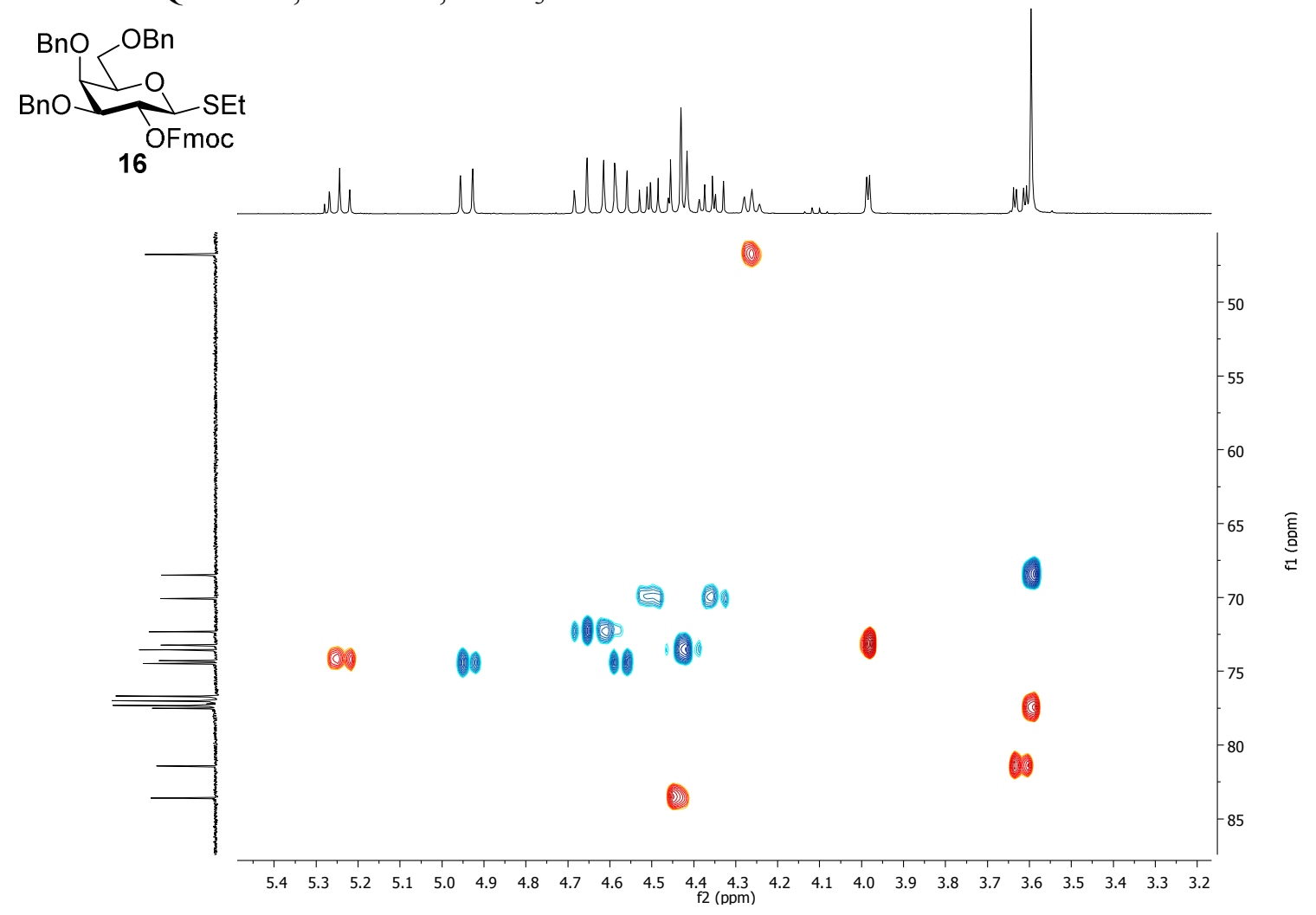

${ }^{1} \mathrm{H}$ NMR, $400 \mathrm{MHz}, \mathrm{CDCl}_{3}$

Fuc9_PROTON_02Mar15_02

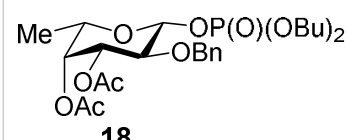

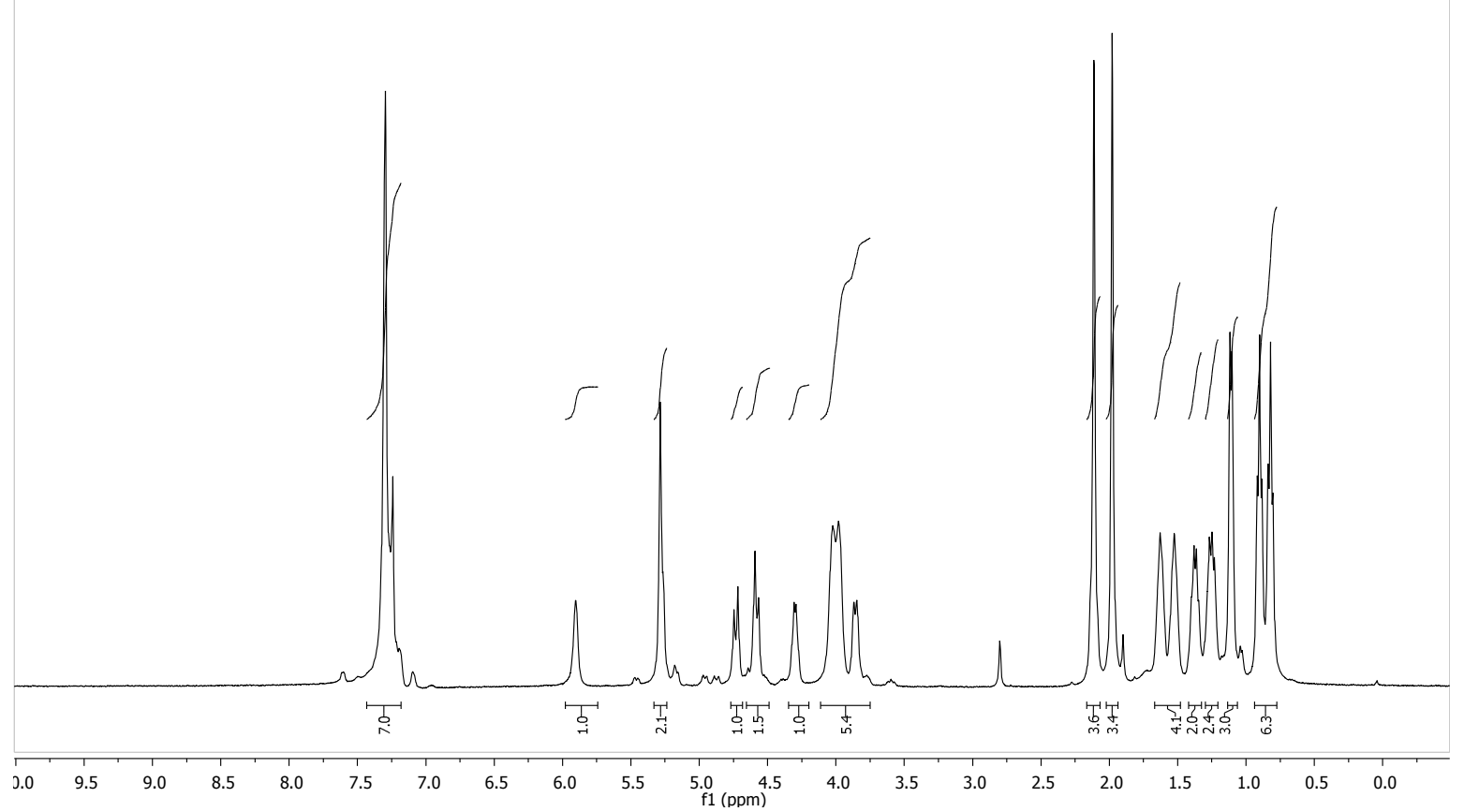


${ }^{13} \mathrm{C}$ NMR, $100 \mathrm{MHz}, \mathrm{CDCl}_{3}$
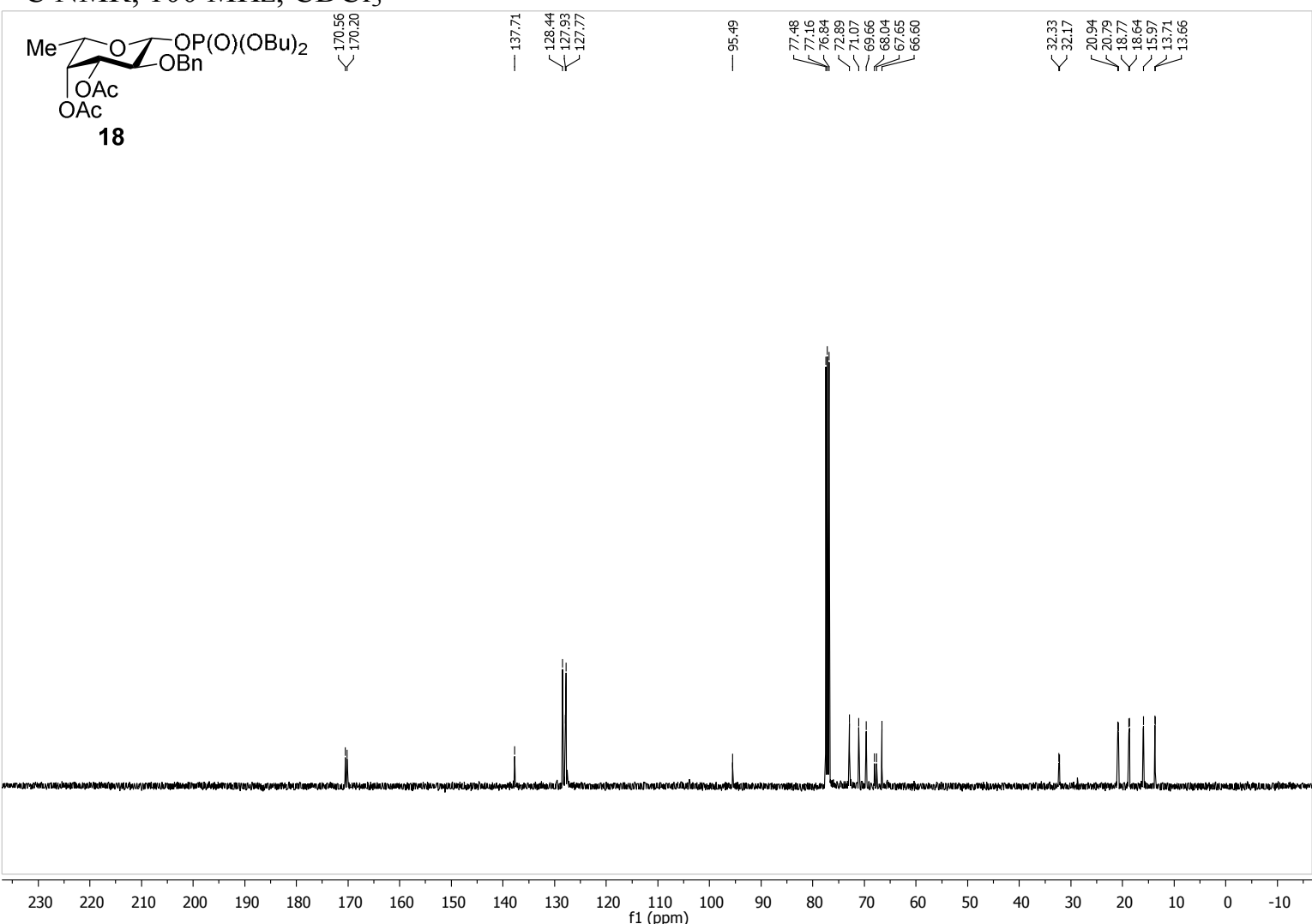

${ }^{1} \mathrm{H}-\mathrm{COSY} \mathrm{NMR}, 400 \mathrm{MHz}, \mathrm{CDCl}_{3}$

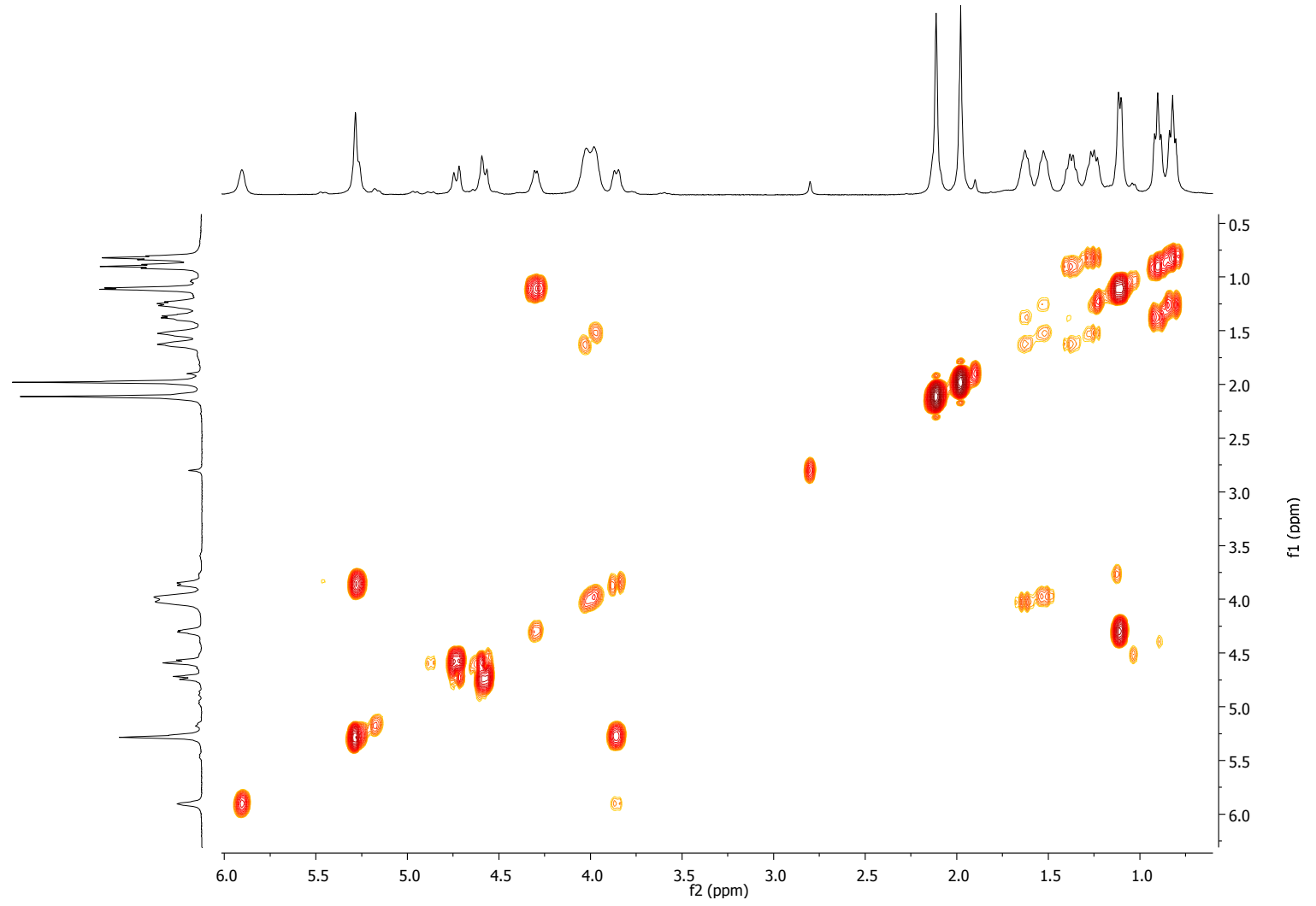


${ }^{1} \mathrm{H}^{-13} \mathrm{C}-\mathrm{HSQC}$ NMR, $400 \mathrm{MHz}, \mathrm{CDCl}_{3}$

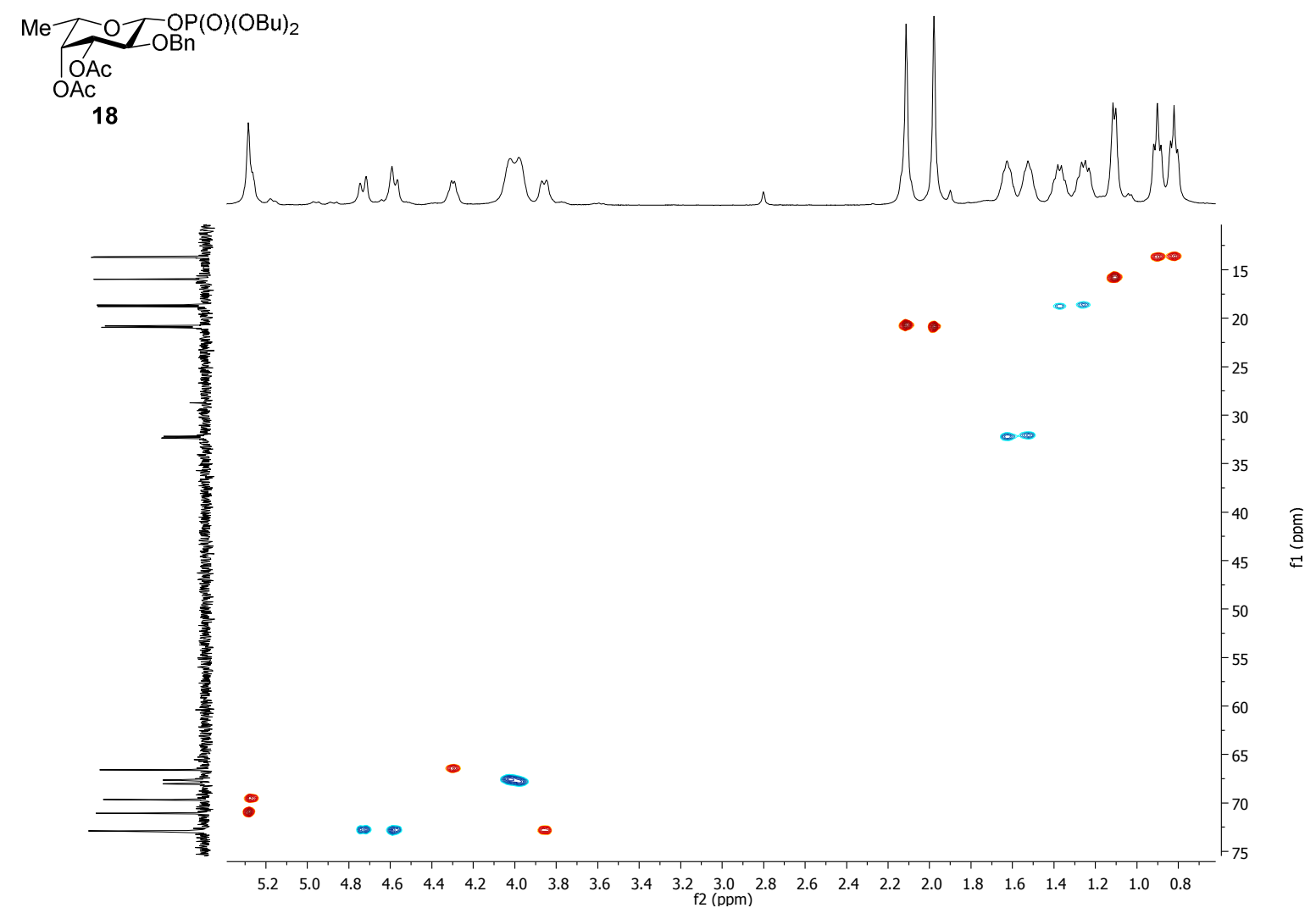

${ }^{31} \mathrm{P}$ NMR, $162 \mathrm{MHz}, \mathrm{CDCl}_{3}$

Fuc9_PHOSPHORUS_02Mar15_01

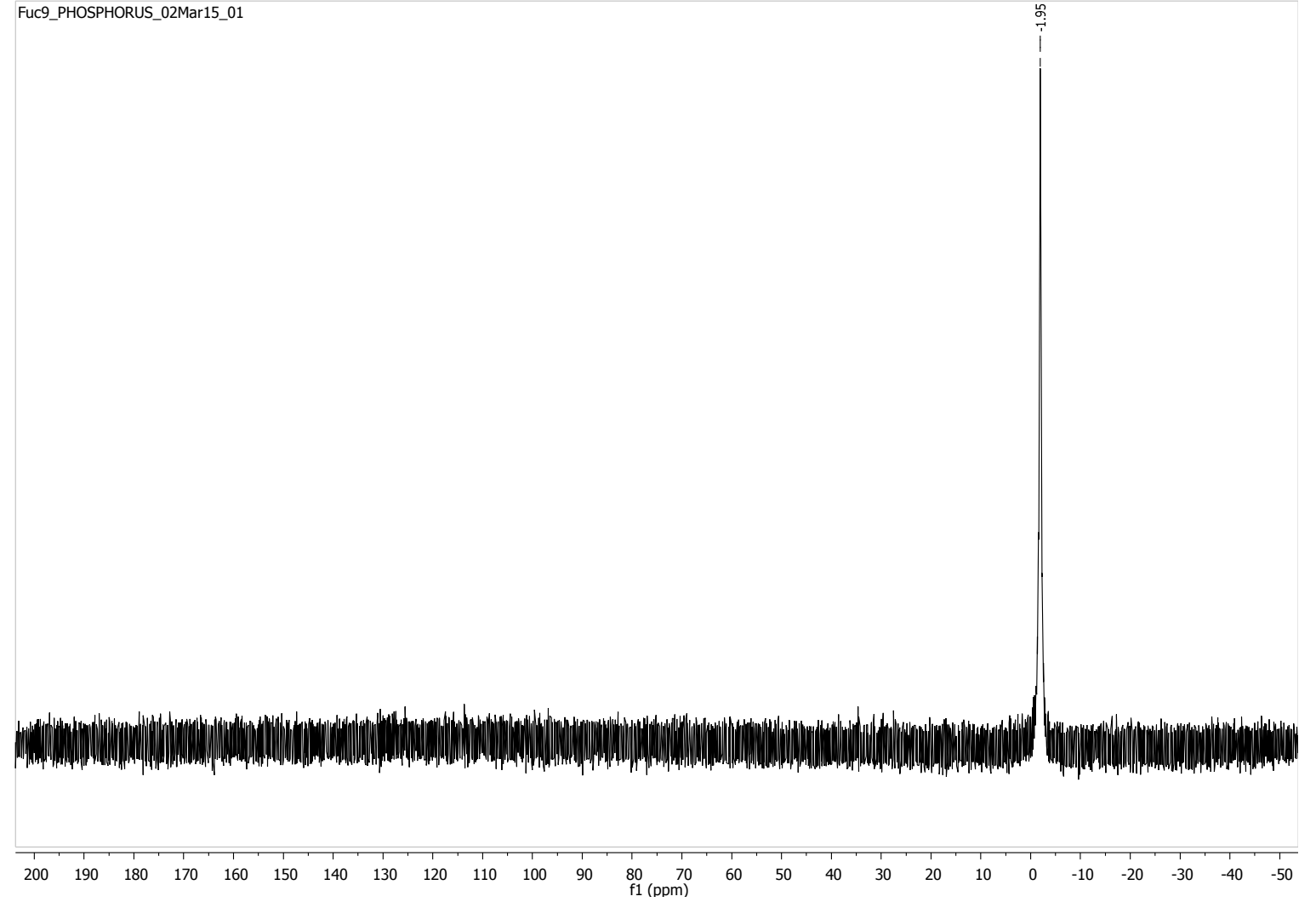


${ }^{1} \mathrm{H} \mathrm{NMR}, 400 \mathrm{MHz}, \mathrm{CDCl}_{3}$

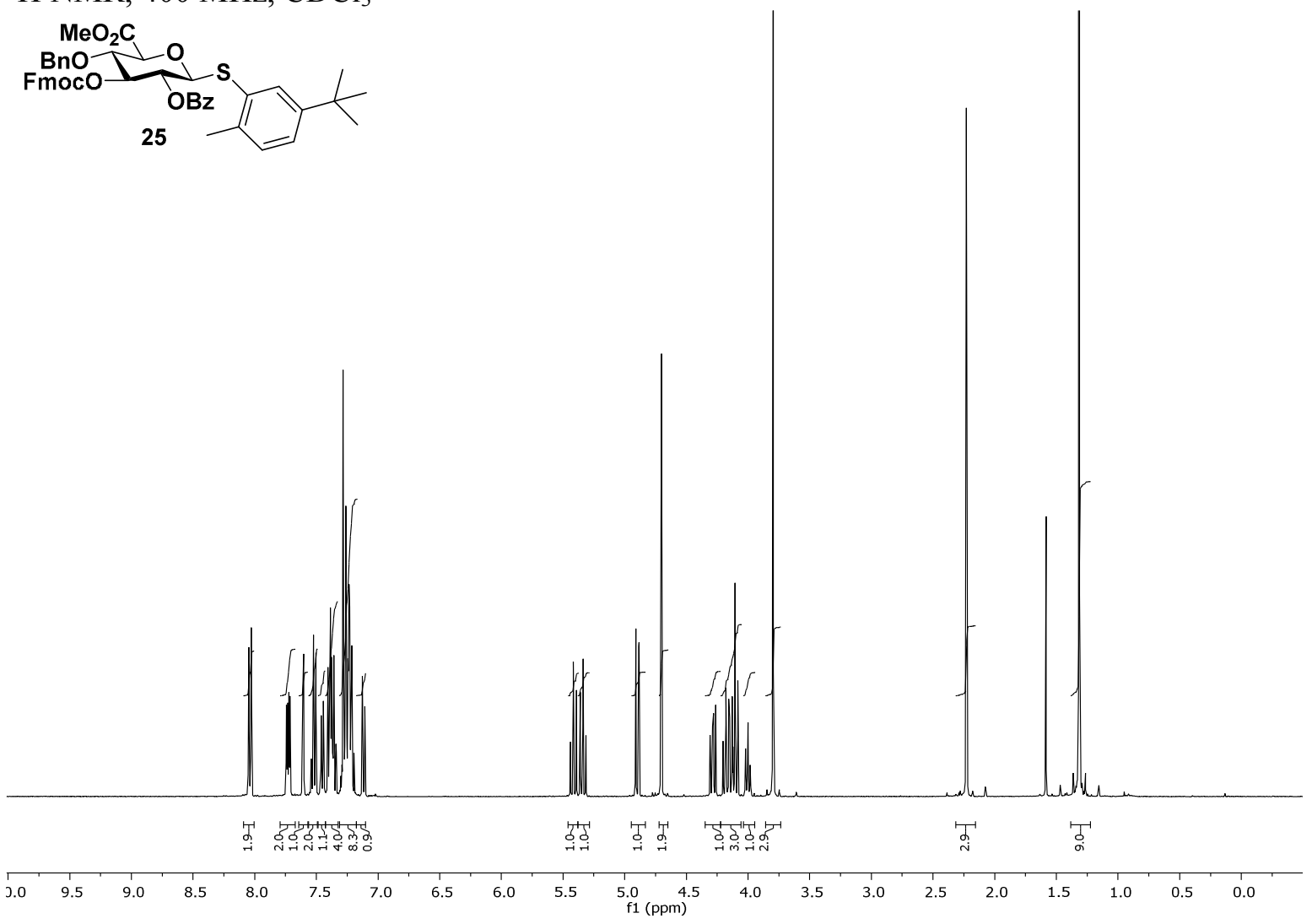

${ }^{13} \mathrm{C} \mathrm{NMR}, 100 \mathrm{MHz}, \mathrm{CDCl}_{3}$
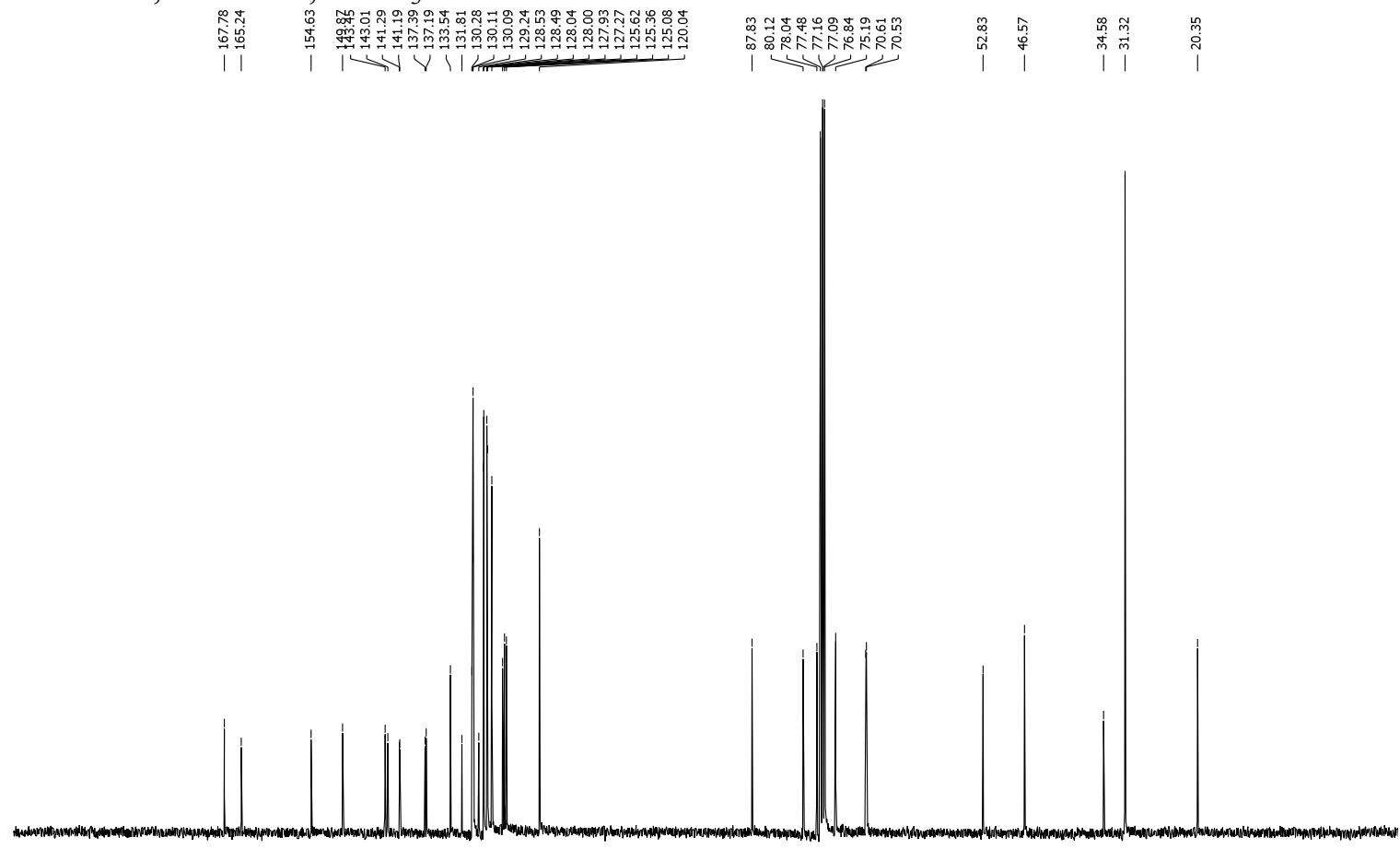

$\begin{array}{llllllllll}190 & 180 & 170 & 160 & 150 & 140 & 130 & 120 & 110 & 100 \\ \mathrm{f} 1(\mathrm{ppm})\end{array}$ 
${ }^{1} \mathrm{H}-\mathrm{COSY} \mathrm{NMR}, 400 \mathrm{MHz}, \mathrm{CDCl}_{3}$

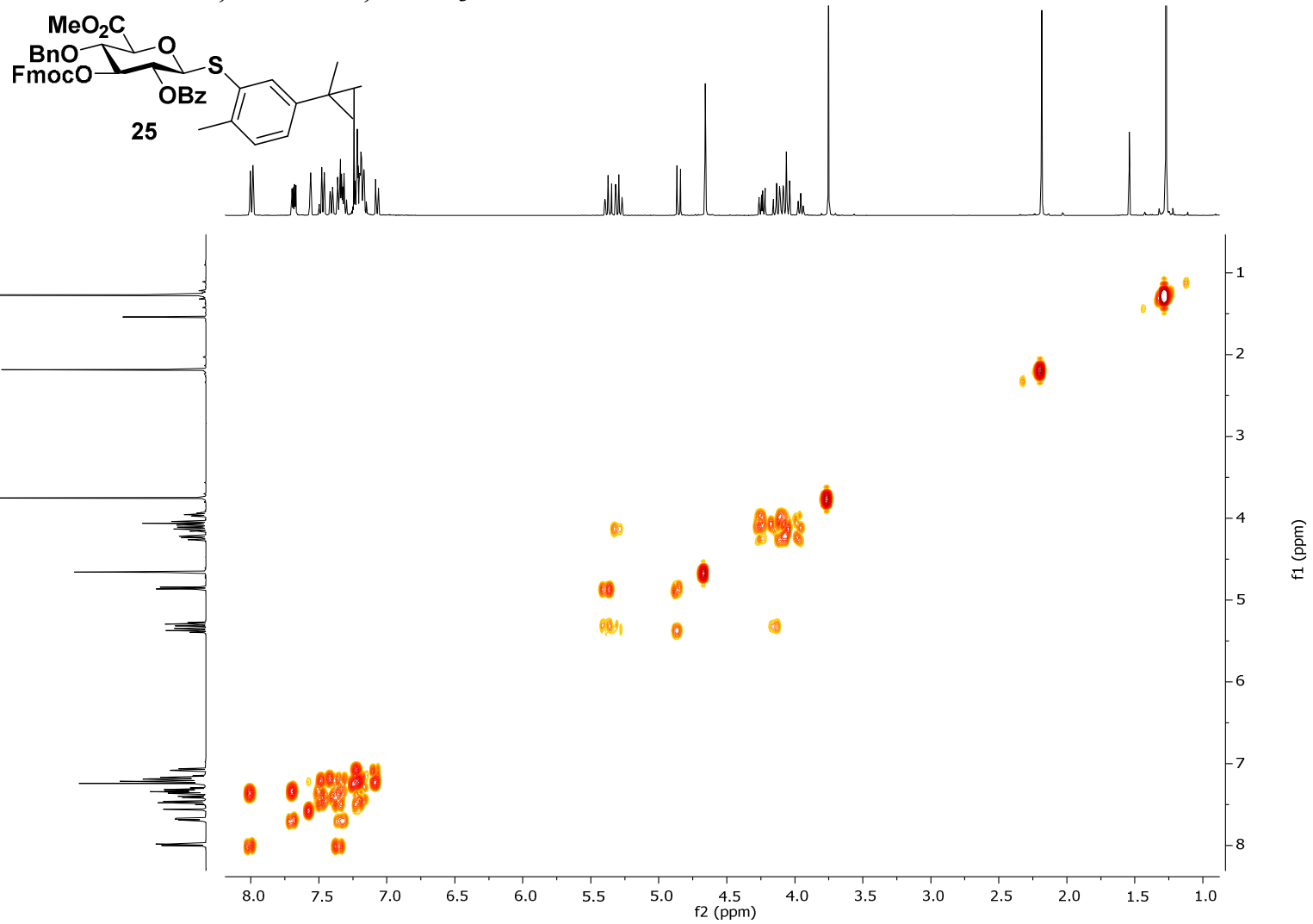

${ }^{1} \mathrm{H}-{ }^{13} \mathrm{C}-\mathrm{HSQC}$ NMR, $400 \mathrm{MHz}, \mathrm{CDCl}_{3}$

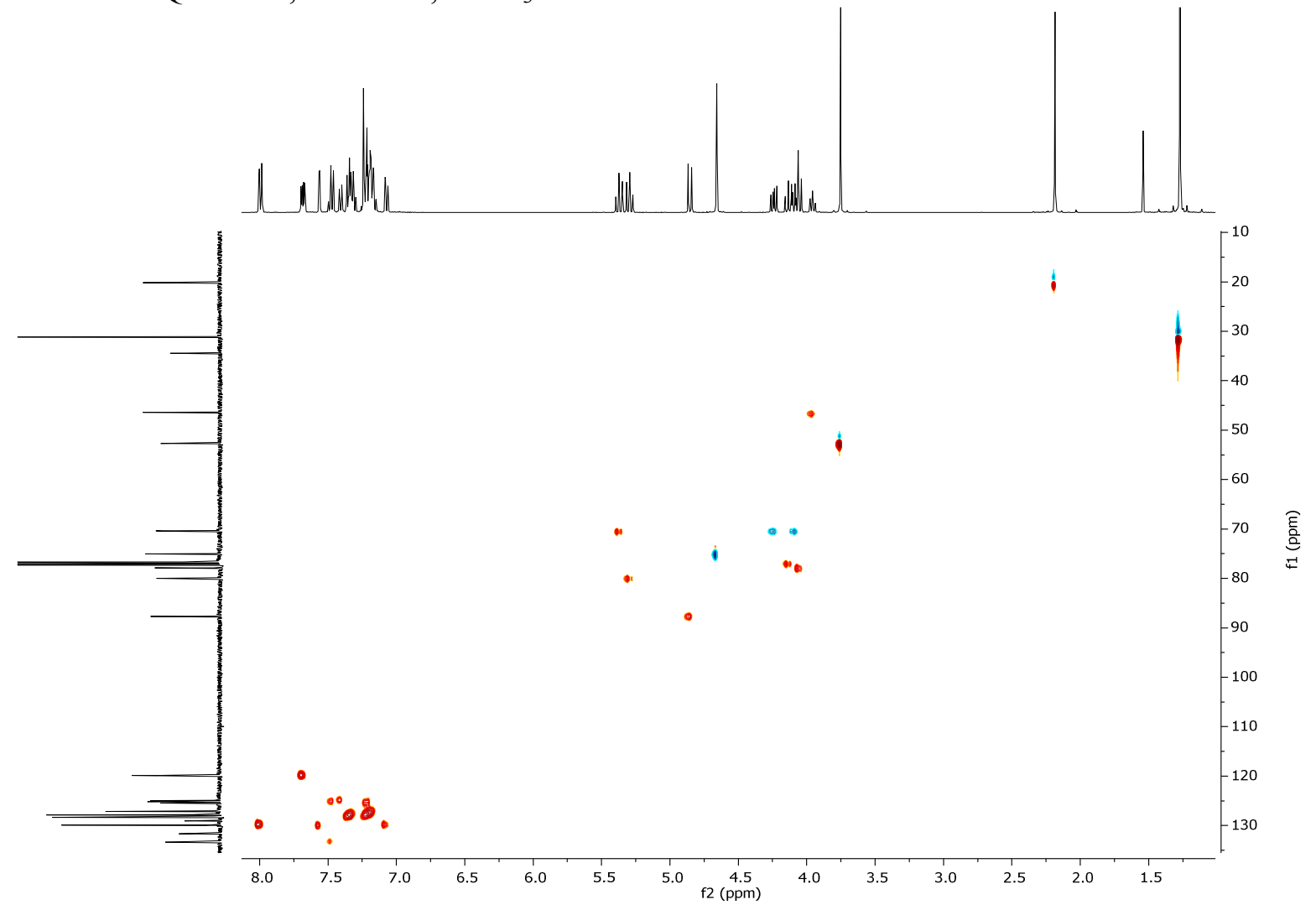


${ }^{1} \mathrm{H}$ NMR, $400 \mathrm{MHz}, \mathrm{CDCl}_{3}$
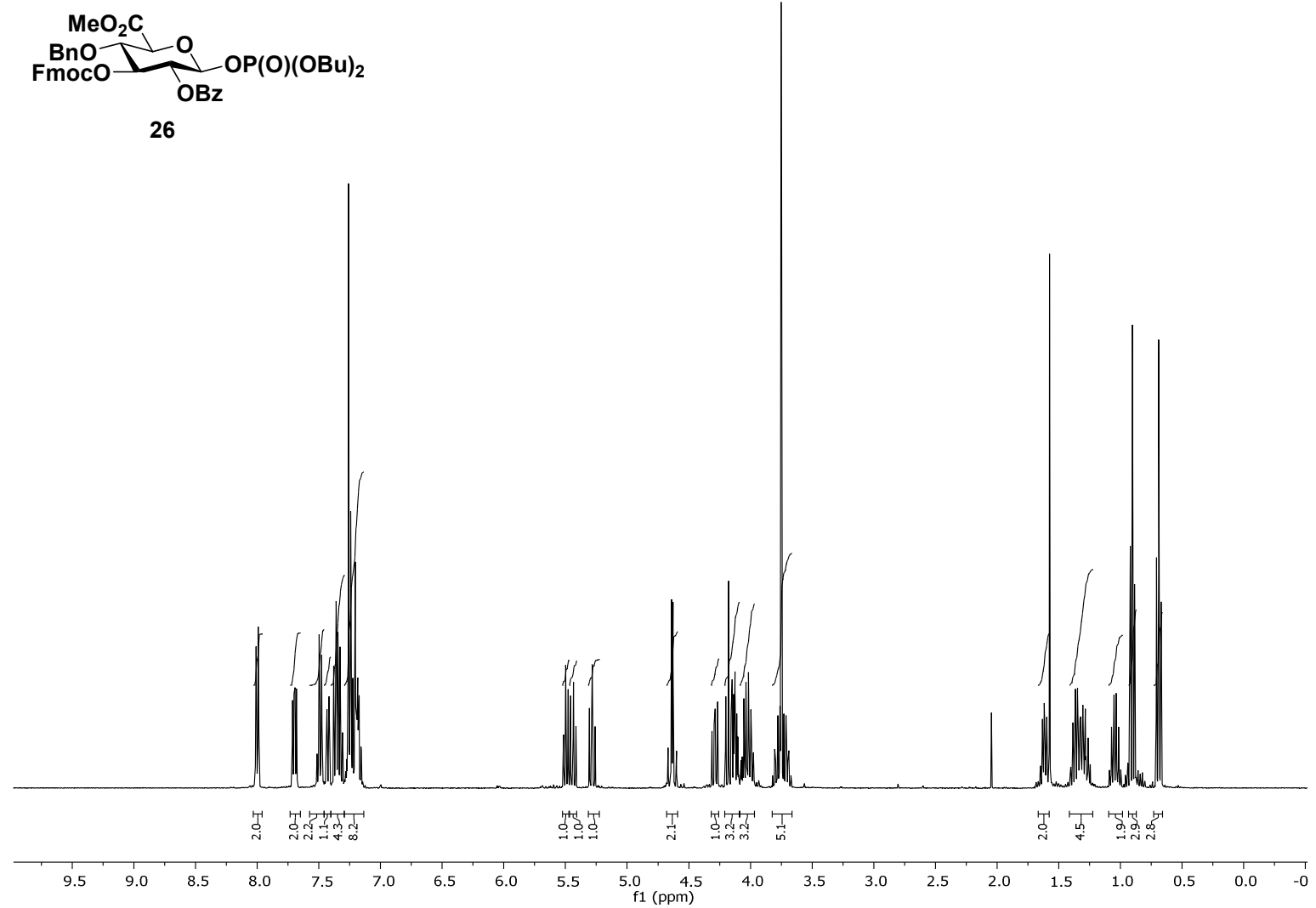

${ }^{13} \mathrm{C} \mathrm{NMR,}, 100 \mathrm{MHz}, \mathrm{CDCl}_{3}$

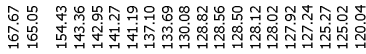

|
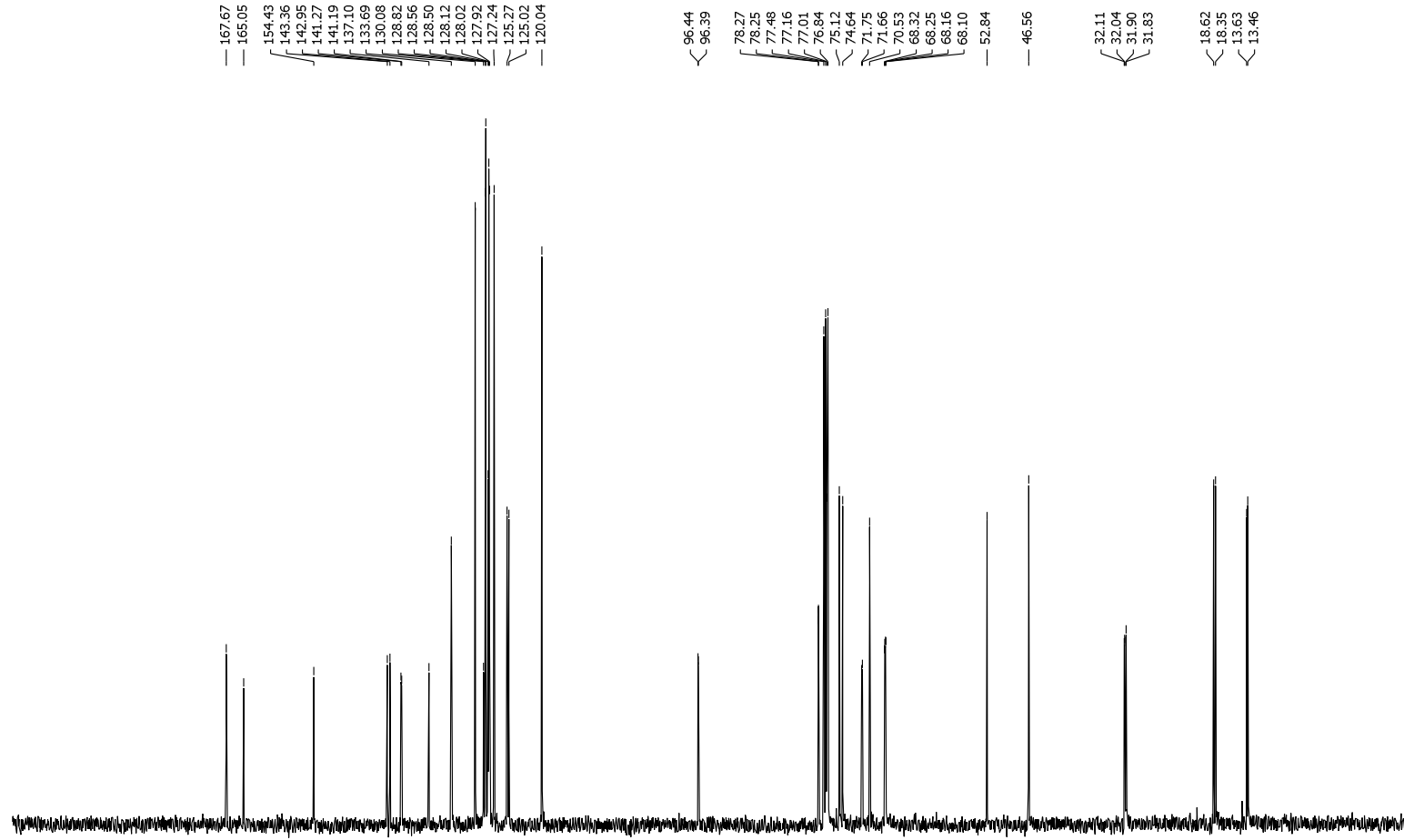

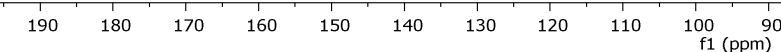

80


${ }^{1} \mathrm{H}-\mathrm{COSY} \mathrm{NMR}, 400 \mathrm{MHz}, \mathrm{CDCl}_{3}$

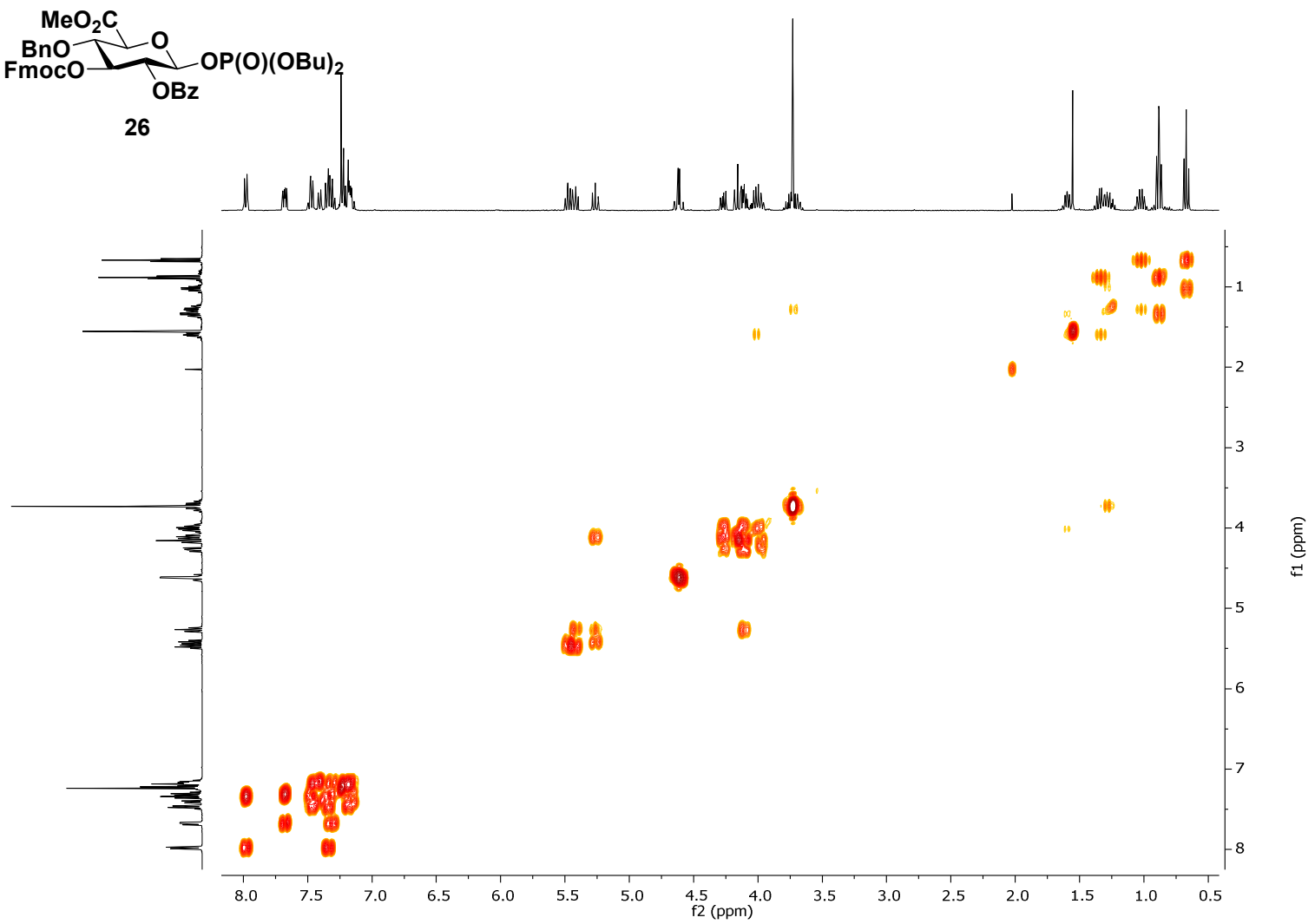

${ }^{1} \mathrm{H}-{ }^{13} \mathrm{C}-\mathrm{HSQC}$ NMR, $400 \mathrm{MHz}, \mathrm{CDCl}_{3}$

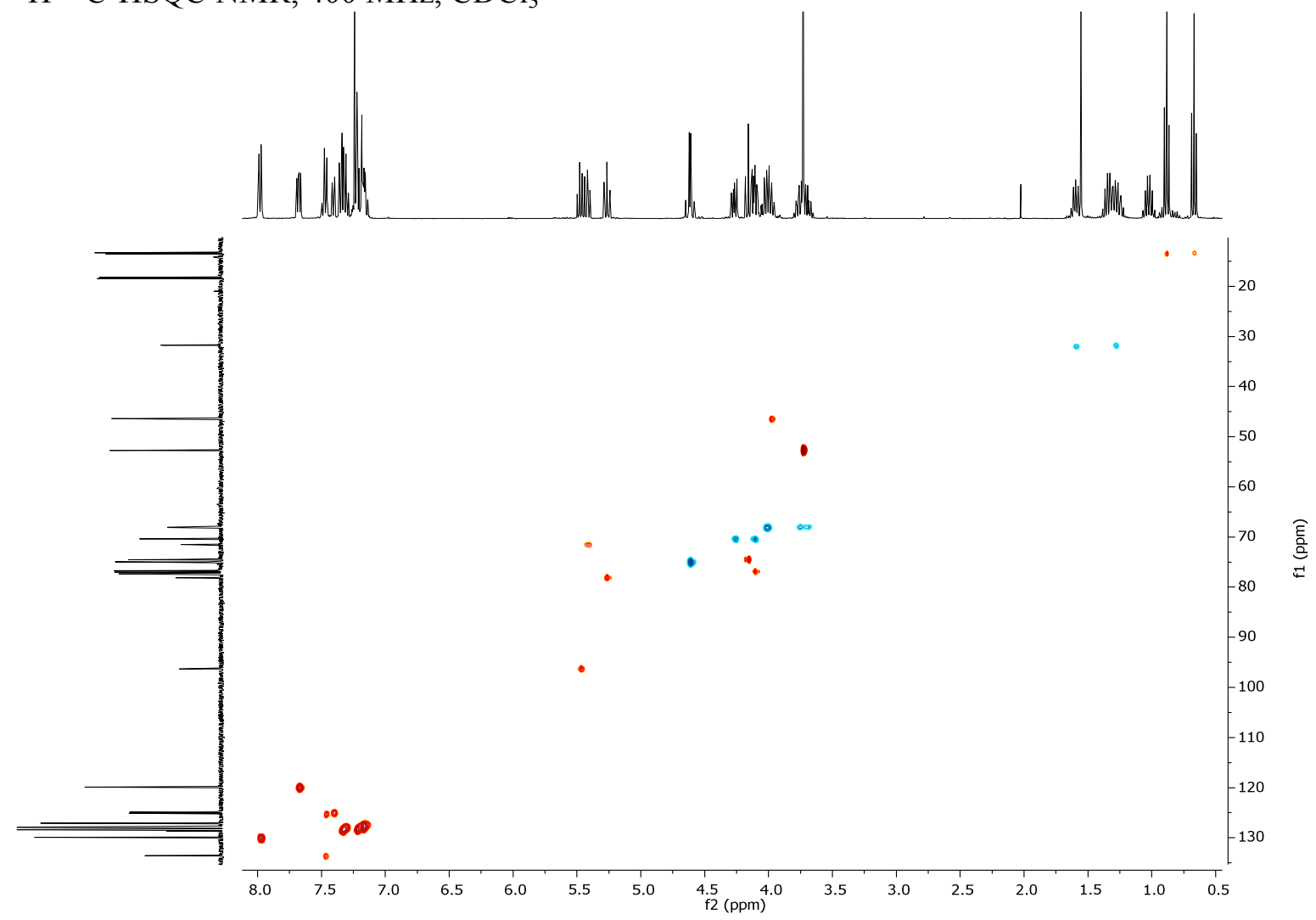


${ }^{31} \mathrm{P}$ NMR, $162 \mathrm{MHz}, \mathrm{CDCl}_{3}$

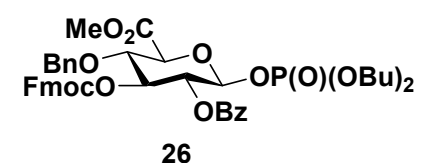

26

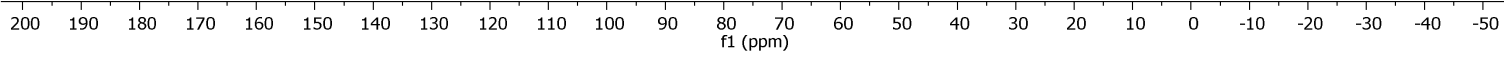

${ }^{1} \mathrm{H}$ NMR, $400 \mathrm{MHz}, \mathrm{CDCl}_{3}$

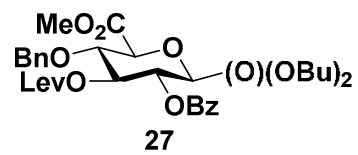

27

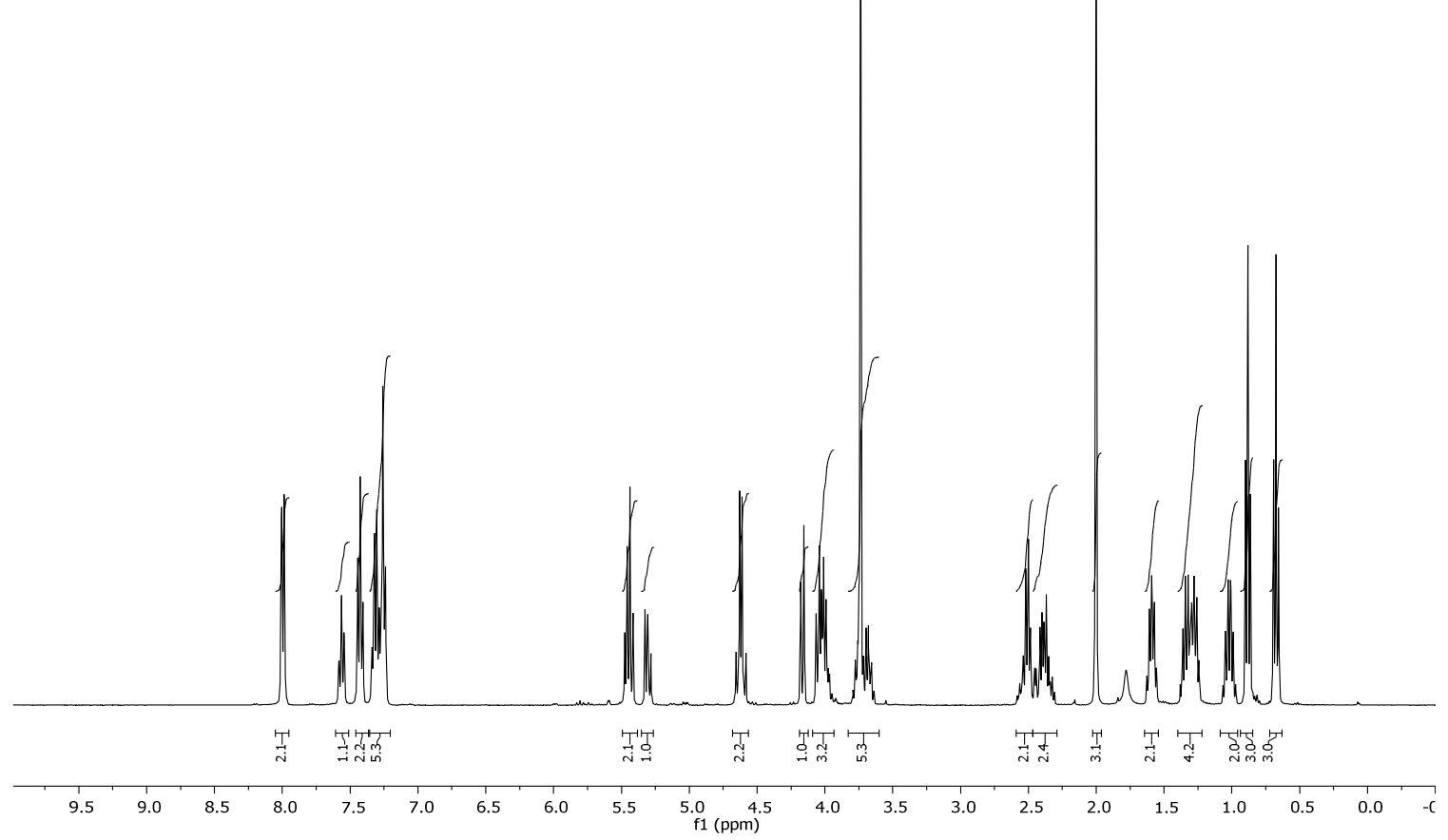


${ }^{13} \mathrm{C} \mathrm{NMR,}, 100 \mathrm{MHz}, \mathrm{CDCl}_{3}$
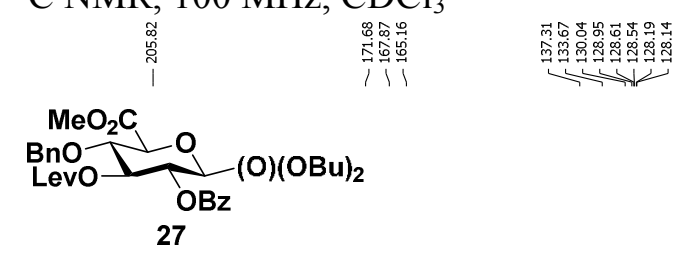

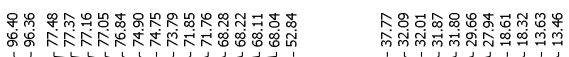

$\checkmark+$
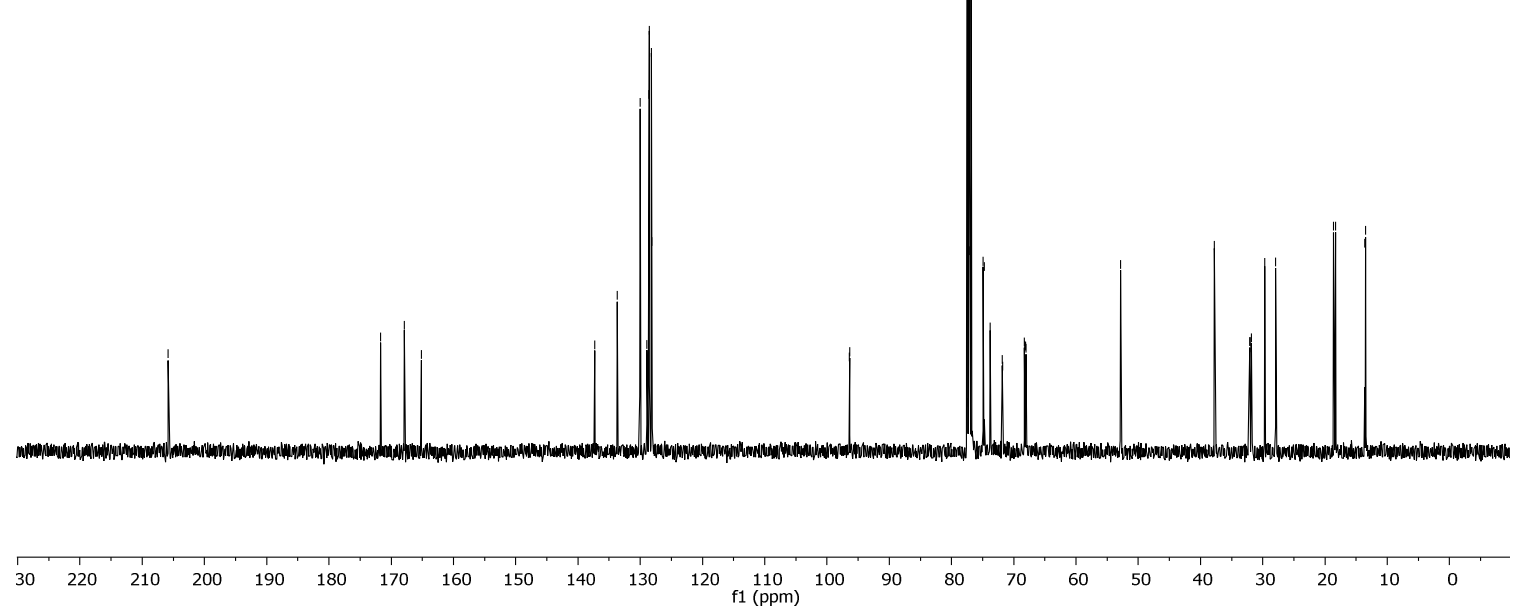

${ }^{1} \mathrm{H}-\mathrm{COSY} \mathrm{NMR}, 400 \mathrm{MHz}, \mathrm{CDCl}_{3}$

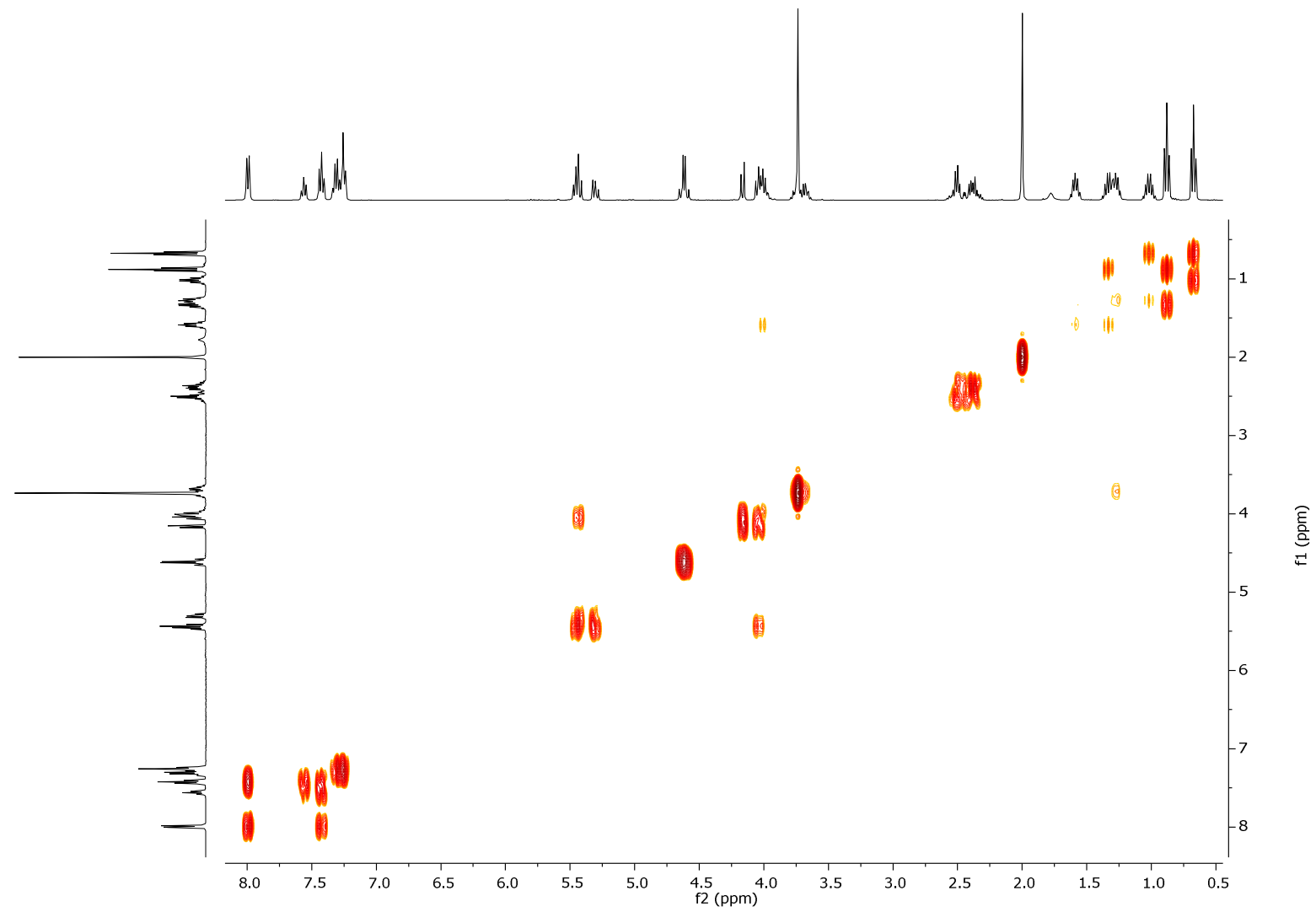


${ }^{1} \mathrm{H}-{ }^{13} \mathrm{C}-\mathrm{HSQC}$ NMR, $400 \mathrm{MHz}, \mathrm{CDCl}_{3}$

$\mathrm{MeO}_{2} \mathrm{C}$

$\underset{\mathrm{OBz}}{\mathrm{BnO} O}(\mathrm{O})(\mathrm{OBu})_{2}$

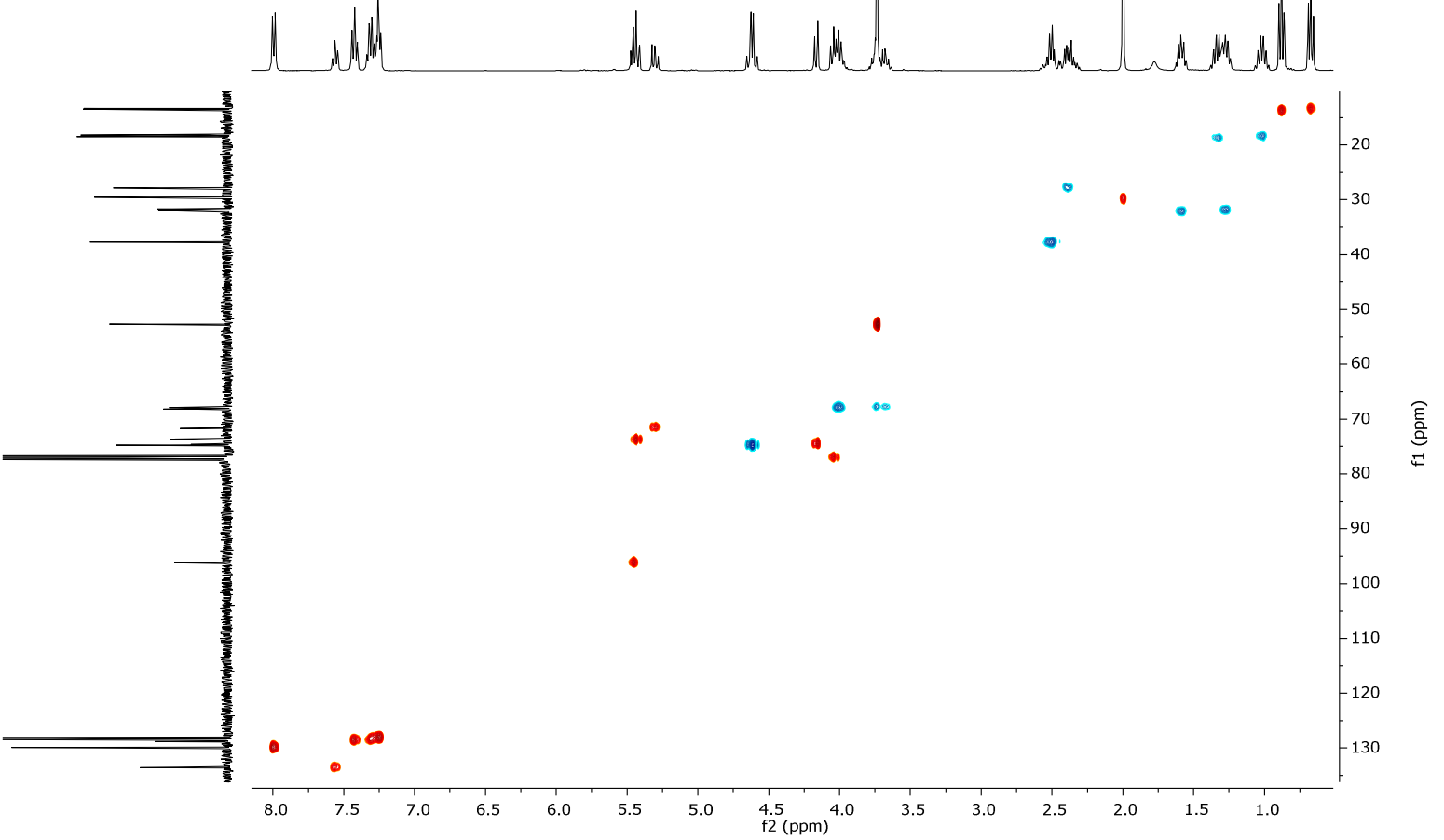

${ }^{31} \mathrm{P} \mathrm{NMR}, 162 \mathrm{MHz}, \mathrm{CDCl}_{3}$

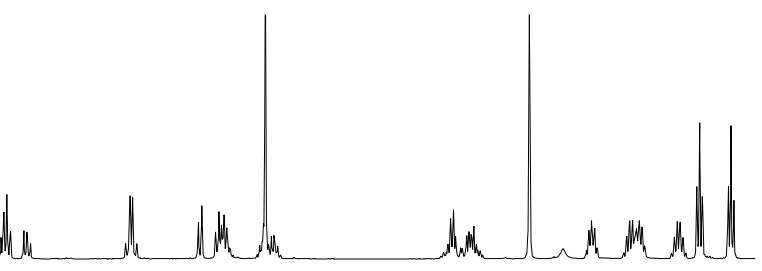

흘$$
\text { (1) }
$$

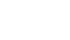




\begin{tabular}{|c|c|c|c|c|c|}
\hline Building Block & Promotor & $T_{\mathrm{a}}\left({ }^{\circ} \mathrm{C}\right)$ & $\mathrm{t}_{1}(\mathrm{~min})$ & $T_{\mathrm{i}}\left({ }^{\circ} \mathrm{C}\right)$ & $\mathrm{t}_{2}(\mathrm{~min})$ \\
\hline 2,6 , and 7 & \multirow{3}{*}{ NIS/TfOH } & -30 & 5 & -10 & 25 \\
\hline $3,8,9,10$, and 11 & & -40 & 5 & -20 & 25 \\
\hline 23 & & -20 & 5 & -10 & 50 \\
\hline 4 and 22 & \multirow{3}{*}{ TMSOTf } & -40 & 5 & -20 & 25 \\
\hline 12 & & -10 & 5 & 0 & 50 \\
\hline 24 & & -20 & 5 & -10 & 50 \\
\hline
\end{tabular}

\begin{tabular}{|c|c|c|c|}
\hline Sequence & Module & Details & Condition \\
\hline \multirow{3}{*}{ I } & 1 & 2.5 eq. of TMSOTf solution & $-20^{\circ} \mathrm{C}$, for $1 \mathrm{~min}$ \\
\hline & 2 & 5 eq. building block $(\mathbf{2}, \mathbf{3}, \mathbf{6}, \mathbf{7}, \mathbf{8}$, and 23$), 5$ eq. of NIS Solution & \\
\hline & 3 & Fmoc Removal & r.t for $5 \mathrm{~min}$ \\
\hline \multirow{3}{*}{ II } & 1 & 2.5 eq. of TMSOTf solution & $-20^{\circ} \mathrm{C}$, for $1 \mathrm{~min}$ \\
\hline & $4-1$ & 5 eq. building block ( 4 and 15 ), 5 eq. of TMSOTf Solution & \\
\hline & 3 & Fmoc Removal & r.t for $5 \mathrm{~min}$ \\
\hline \multirow{2}{*}{ III } & 1 & 2.5 eq. of TMSOTf solution & $-20^{\circ} \mathrm{C}$, for $1 \mathrm{~min}$ \\
\hline & 2 & 5 eq. building block $(9,10$, and 11) 5 eq. of NIS Solution & \\
\hline \multirow{2}{*}{ III } & 1 & 2.5 eq. of TMSOTf solution & $-20^{\circ} \mathrm{C}$, for $1 \mathrm{~min}$ \\
\hline & 2 & 5 eq. building block (22), 5 eq. of TMSOTf solution & \\
\hline \multirow{3}{*}{ IV } & 1 & 2.5 eq. of TMSOTf solution & $-20^{\circ} \mathrm{C}$, for $1 \mathrm{~min}$ \\
\hline & $4-1$ & 5 eq. building block $\mathbf{1 2}$ and $\mathbf{2 4 , 5}$ eq. of TMSOTf Solution & \\
\hline & 5 & Lev Removal & r.t for $5 \mathrm{~min}$ \\
\hline \multirow{3}{*}{$\mathrm{V}$} & 1 & 2.5 eq. of TMSOTf solution & $-20^{\circ} \mathrm{C}$, for $1 \mathrm{~min}$ \\
\hline & $4-2$ & 5 eq. building block $\mathbf{1 2}, 5$ eq. of TMSOTf Solution & \\
\hline & 5 & Lev Removal & r.t for $5 \mathrm{~min}$ \\
\hline
\end{tabular}

Table S1. Sequences of the glycosylation cycle with the corresponding monomers and optimized conditions for "approved building block". Glycosylation condition: activation temperature $\left(\boldsymbol{T}_{\mathrm{a}}\right)$ and time $\left(\mathrm{t}_{1}\right)$, incubation temperature $\left(\boldsymbol{T}_{\mathrm{i}}\right)$ and time $\left(\mathrm{t}_{2}\right)$. 


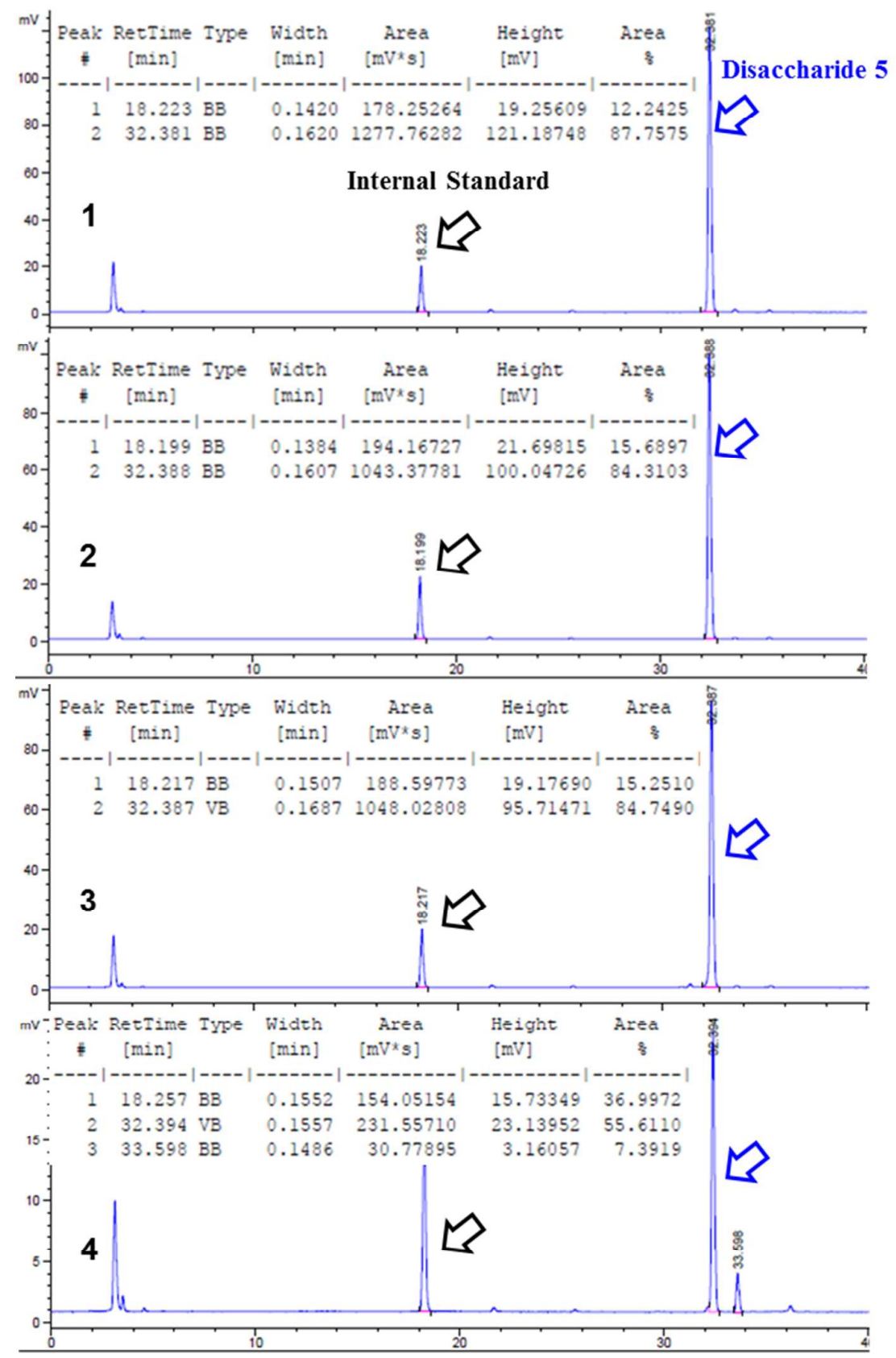

Figure S1. LC-MS of disaccharide 13 (blue arrow) including building block 11 (black arrow) as an Internal standard. 
${ }^{1} \mathrm{H} \mathrm{NMR}, 400 \mathrm{MHz}, \mathrm{CDCl}_{3}$

HHS-Auto-250-ST2-2_PROTON_01

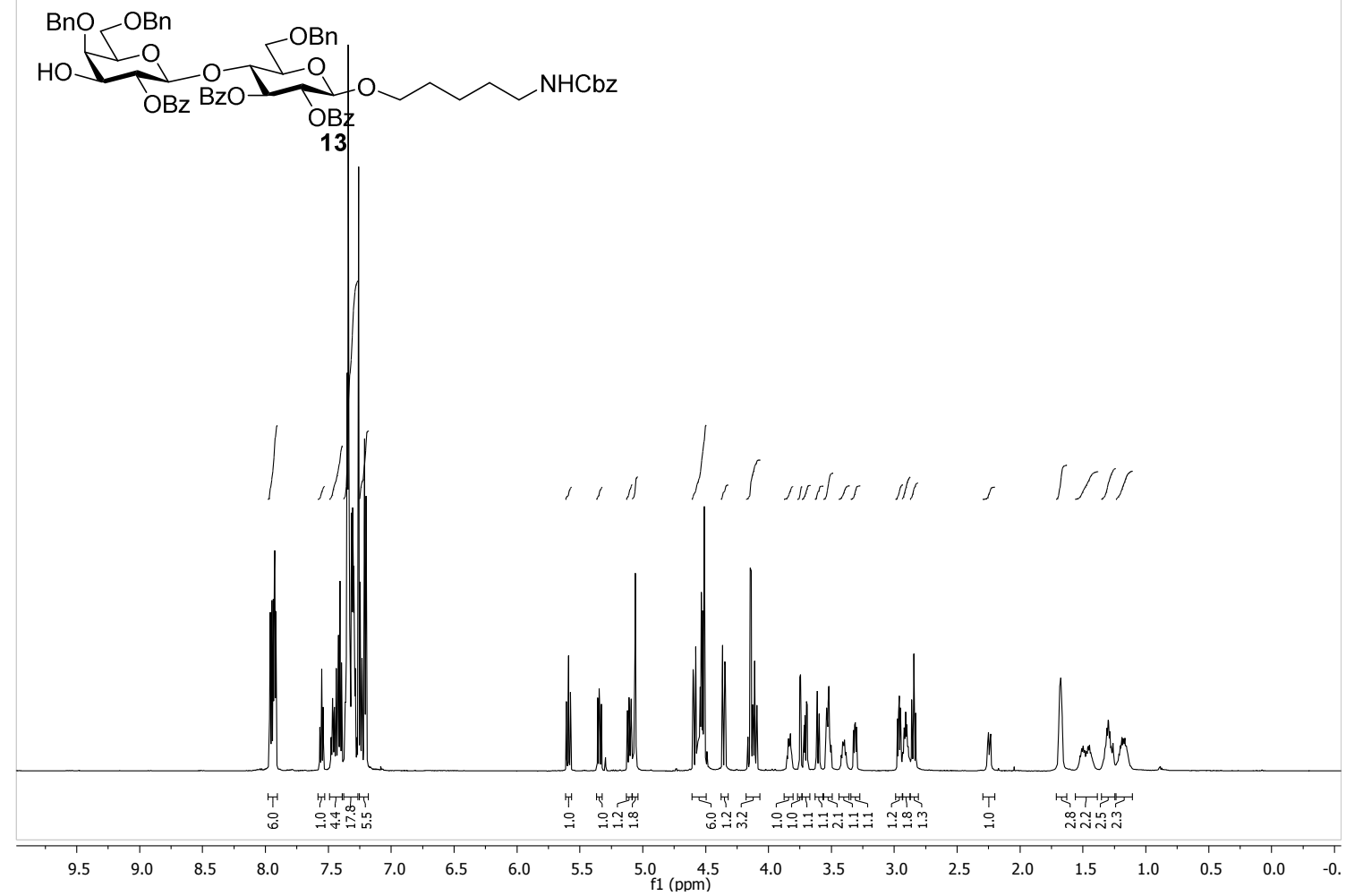

${ }^{13} \mathrm{C} \mathrm{NMR,}, 100 \mathrm{MHz}, \mathrm{CDCl}_{3}$

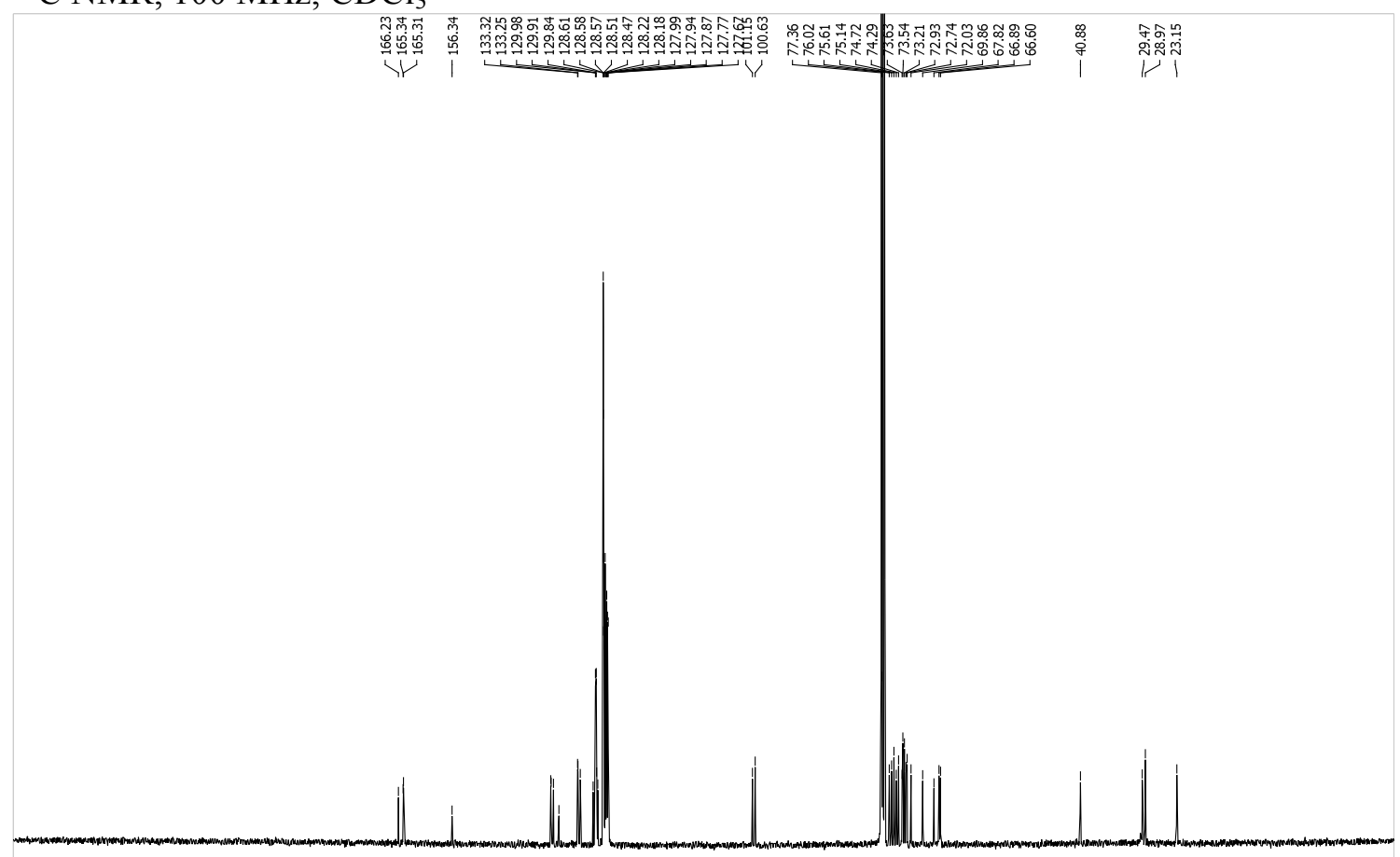

$\begin{array}{lllllllllllllllllllllllllllll}230 & 220 & 210 & 200 & 190 & 180 & 170 & 160 & 150 & 140 & 130 & 120 & \begin{array}{c}110 \\ \mathrm{f} 1(\mathrm{ppm})\end{array} & 100 & 90 & 80 & 70 & 60 & 50 & 40 & 30 & 20 & 10 & 0 & -10\end{array}$ 
${ }^{1} \mathrm{H}-\mathrm{COSY} \mathrm{NMR}, 400 \mathrm{MHz}, \mathrm{CDCl}_{3}$

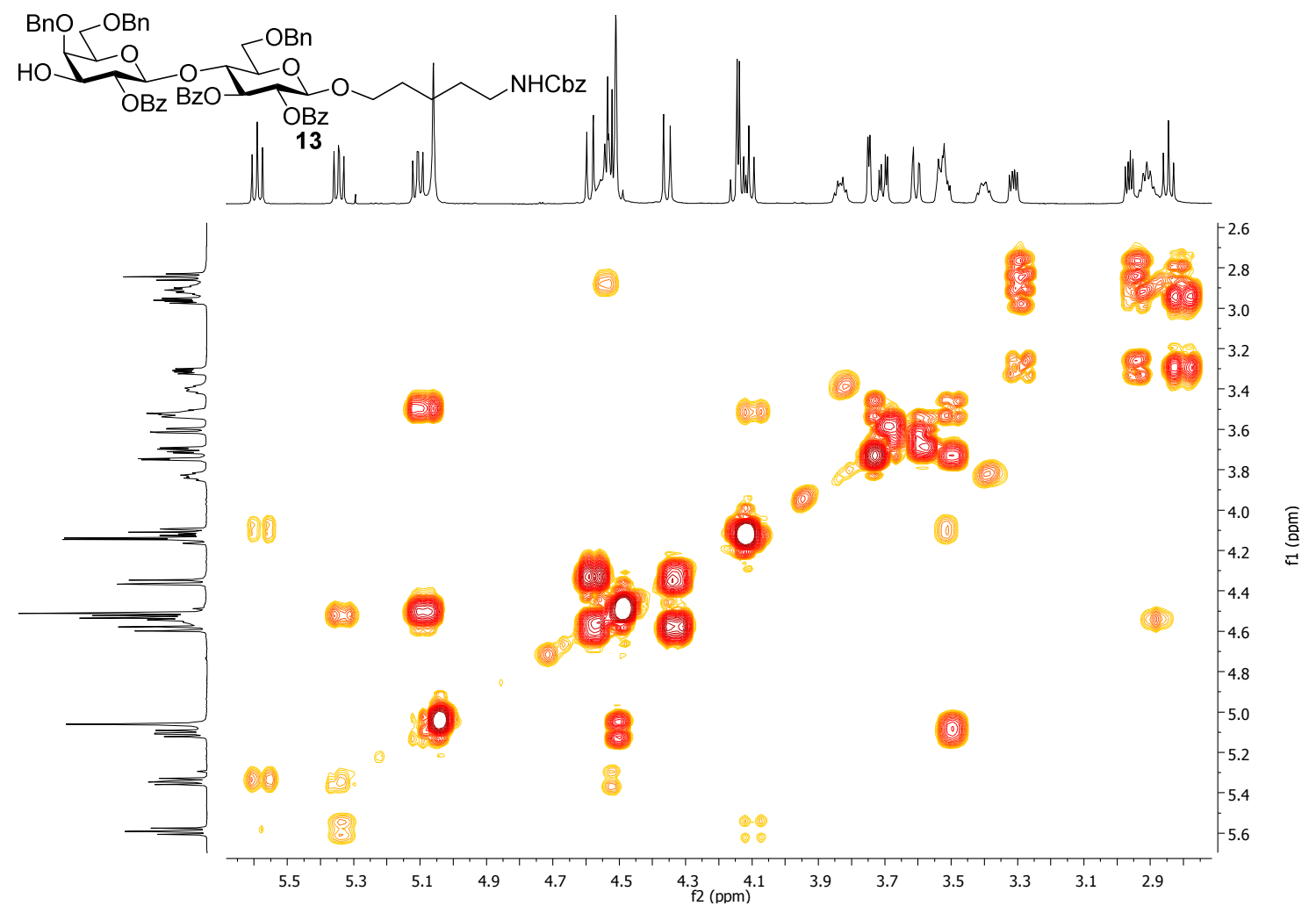

${ }^{1} \mathrm{H}_{-}{ }^{13} \mathrm{C}-\mathrm{HSQC}$ NMR, $400 \mathrm{MHz}, \mathrm{CDCl}_{3}$

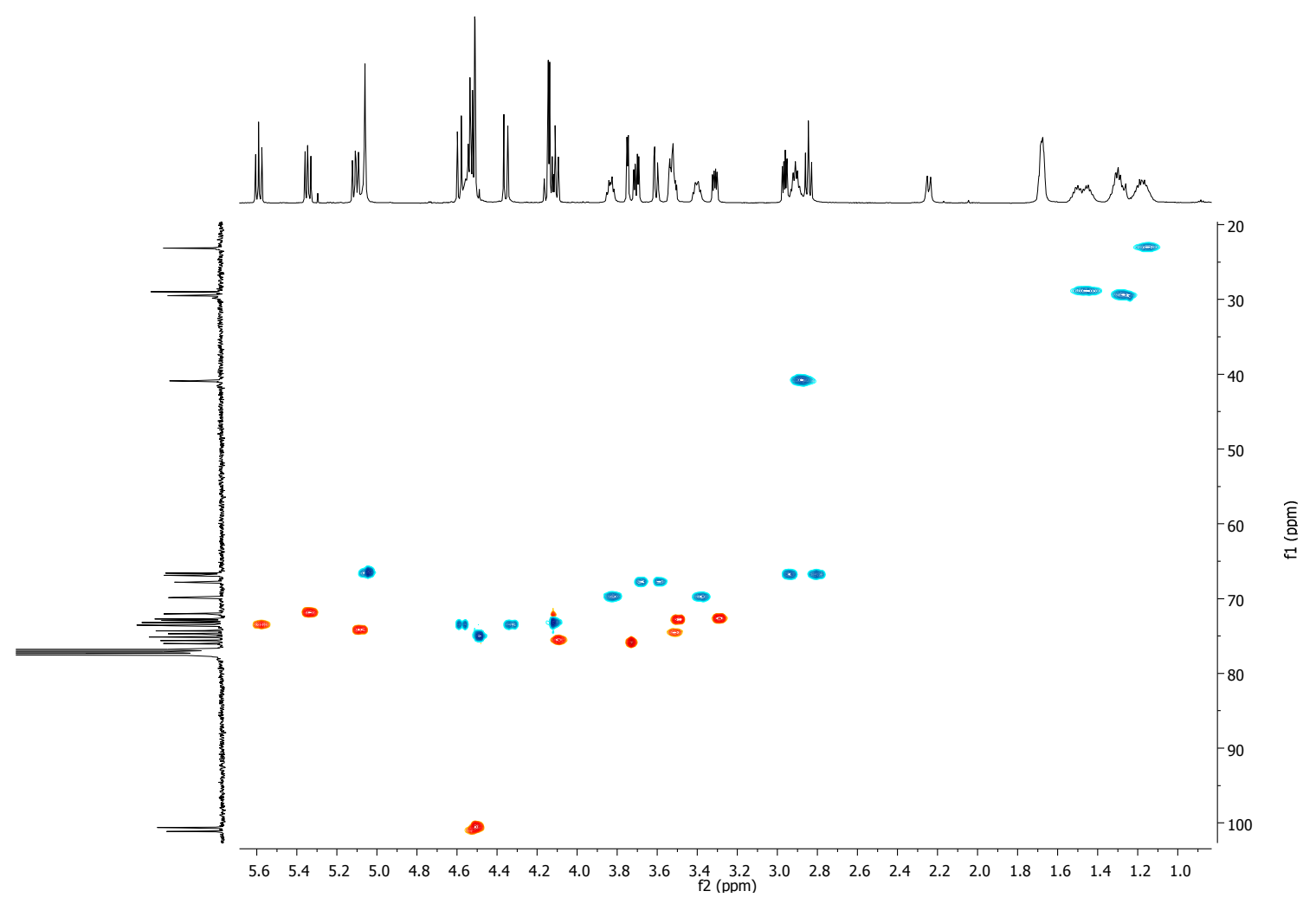


${ }^{1} \mathrm{H} \mathrm{NMR}, 600 \mathrm{MHz}, \mathrm{CDCl}_{3}$

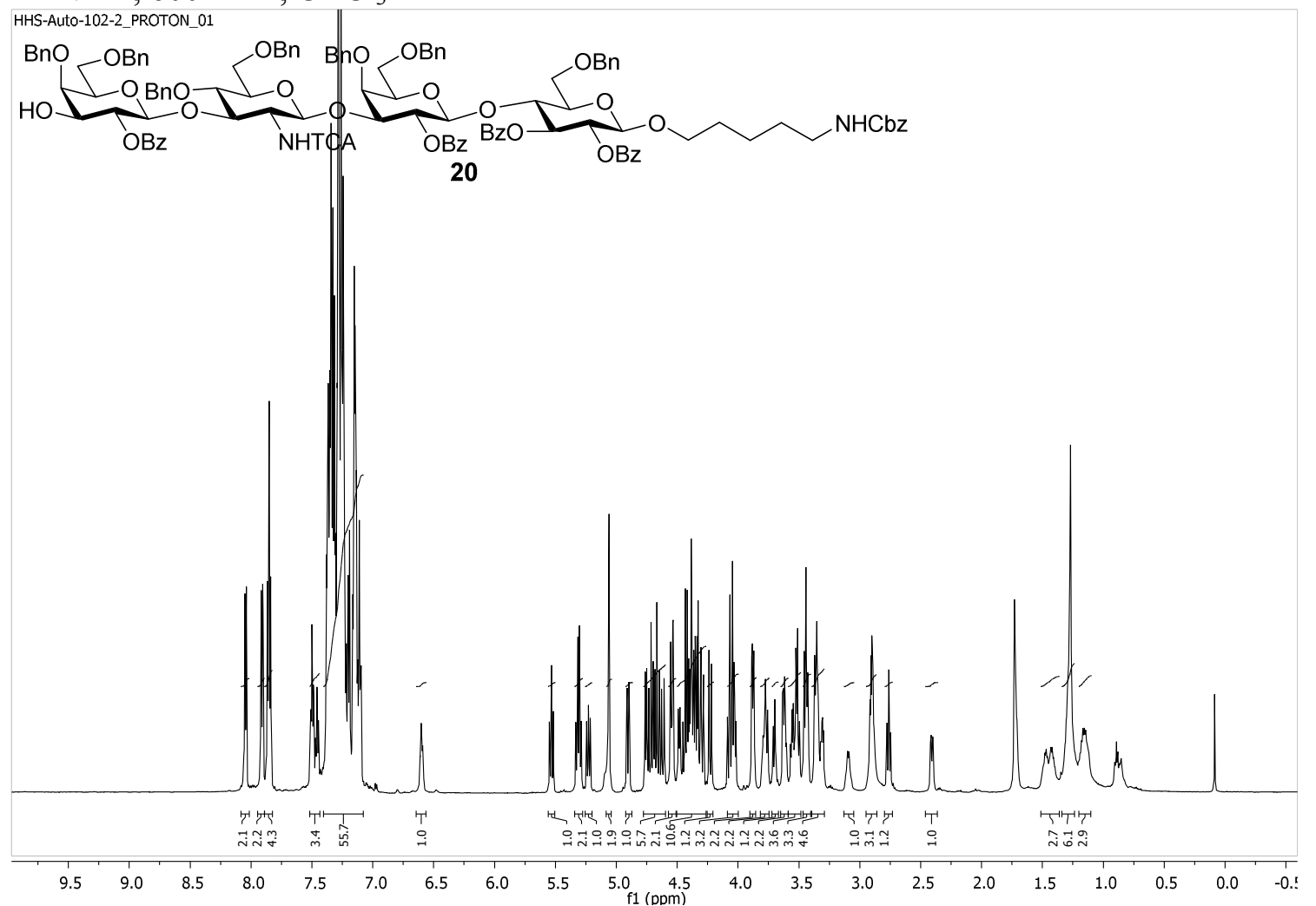

${ }^{13} \mathrm{C}$ NMR, $150 \mathrm{MHz}, \mathrm{CDCl}_{3}$
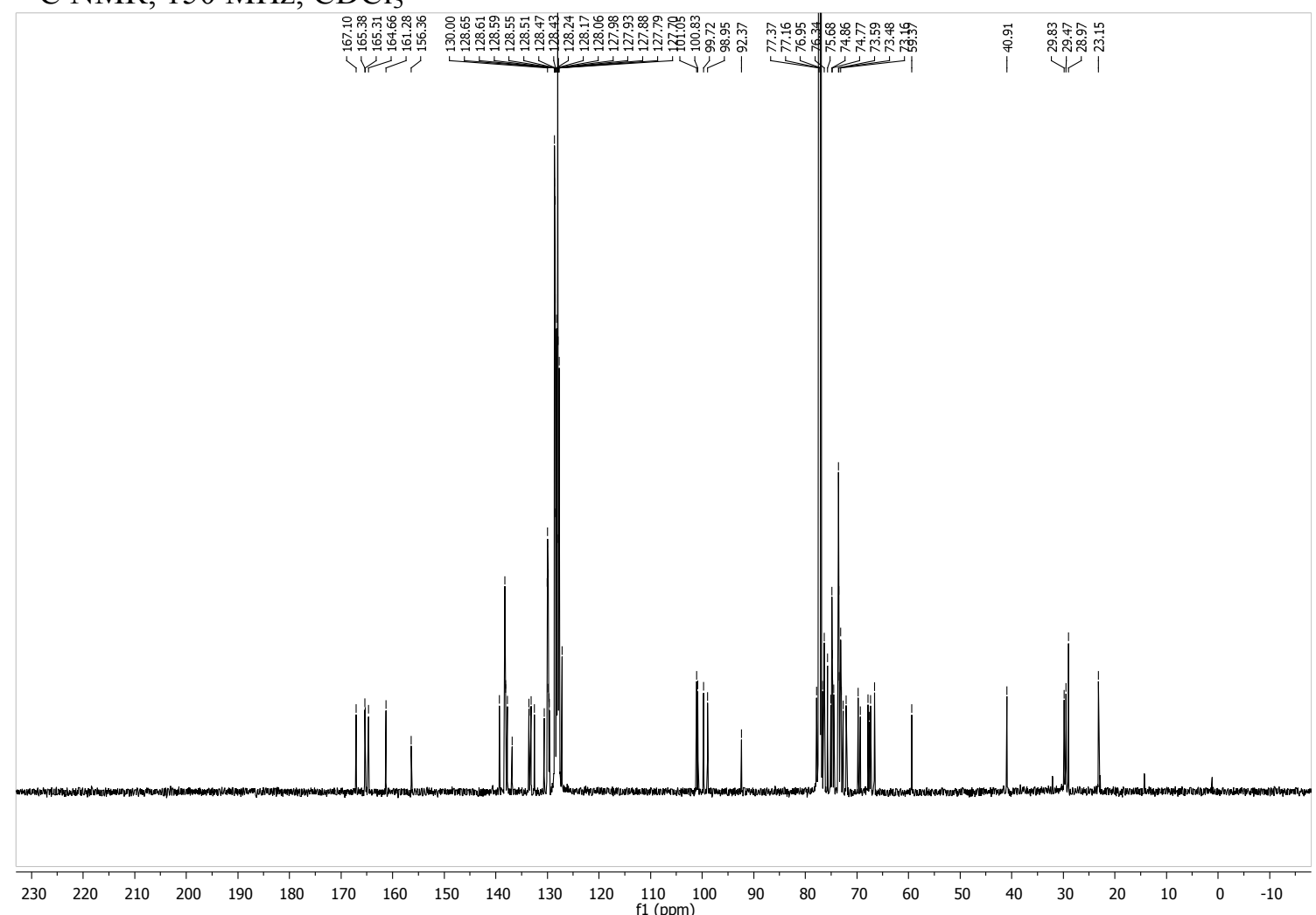
${ }^{1} \mathrm{H}-\mathrm{COSY} \mathrm{NMR}, 600 \mathrm{MHz}, \mathrm{CDCl}_{3}$

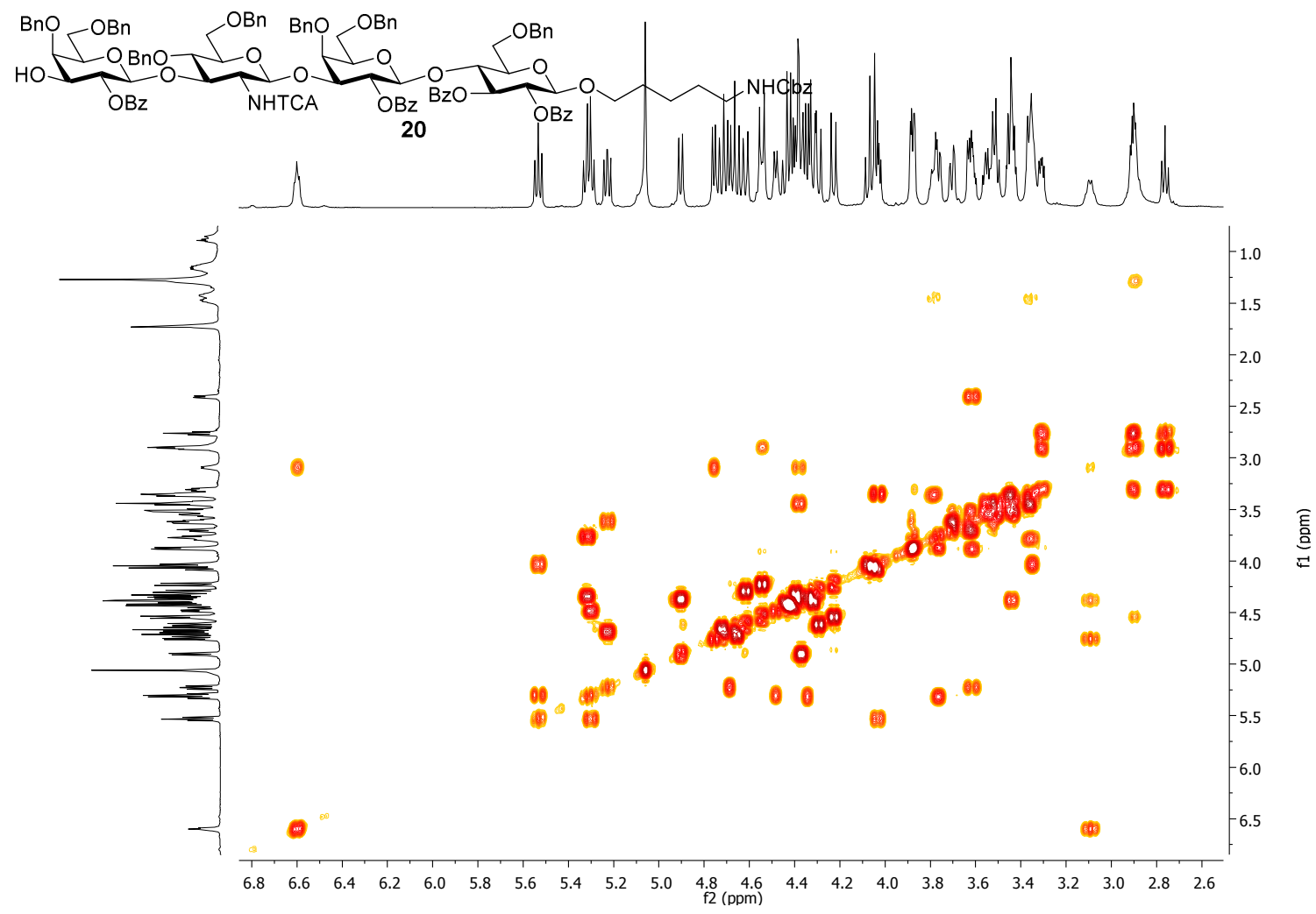

${ }^{1} \mathrm{H}_{-}{ }^{13} \mathrm{C}-\mathrm{HSQC}$ NMR, $600 \mathrm{MHz}, \mathrm{CDCl}_{3}$

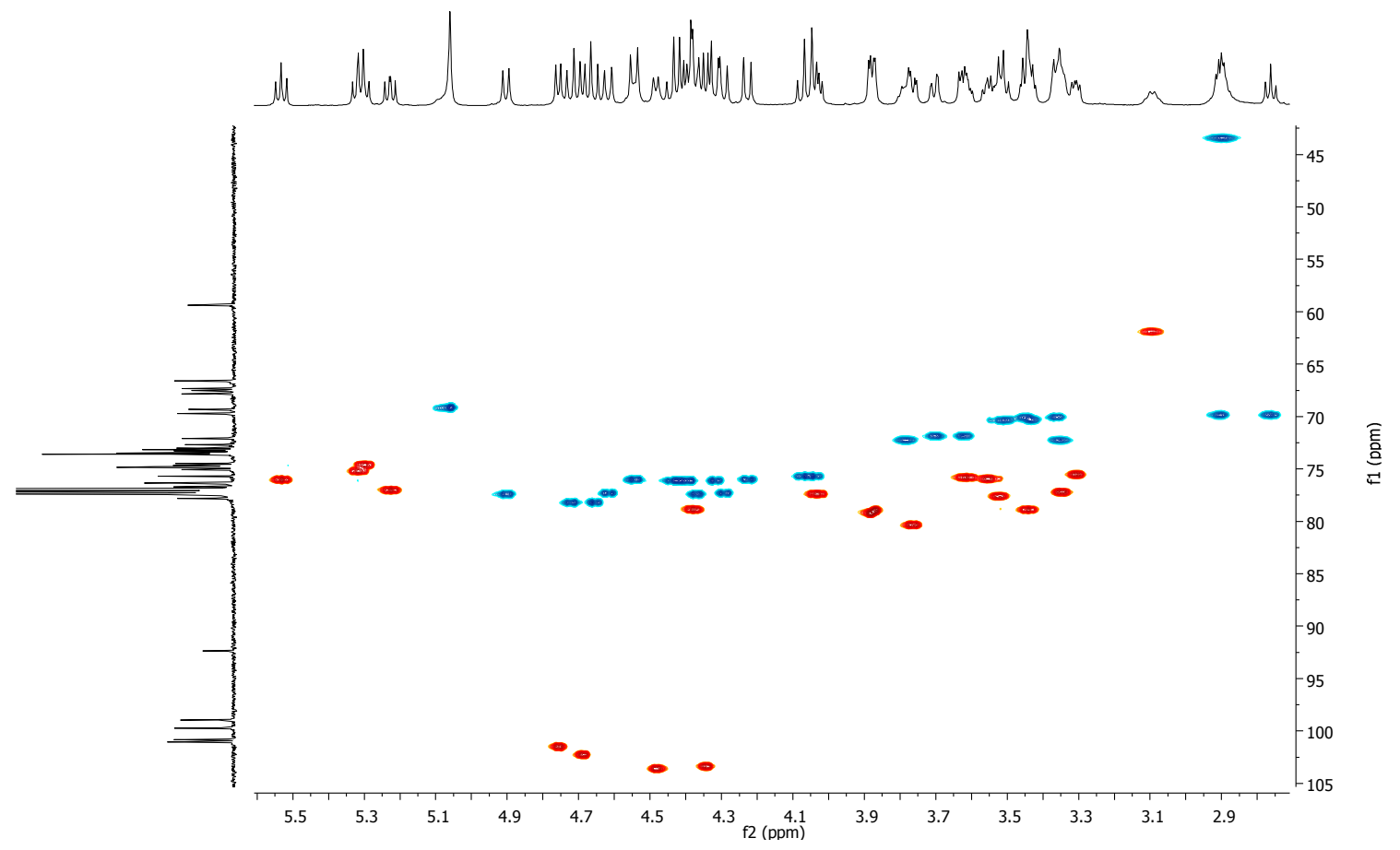


${ }^{1} \mathrm{H} \mathrm{NMR}, 600 \mathrm{MHz}, \mathrm{CDCl}_{3}$

HHS-Auto-101-2-Purified_PROTON_23Feb13_01
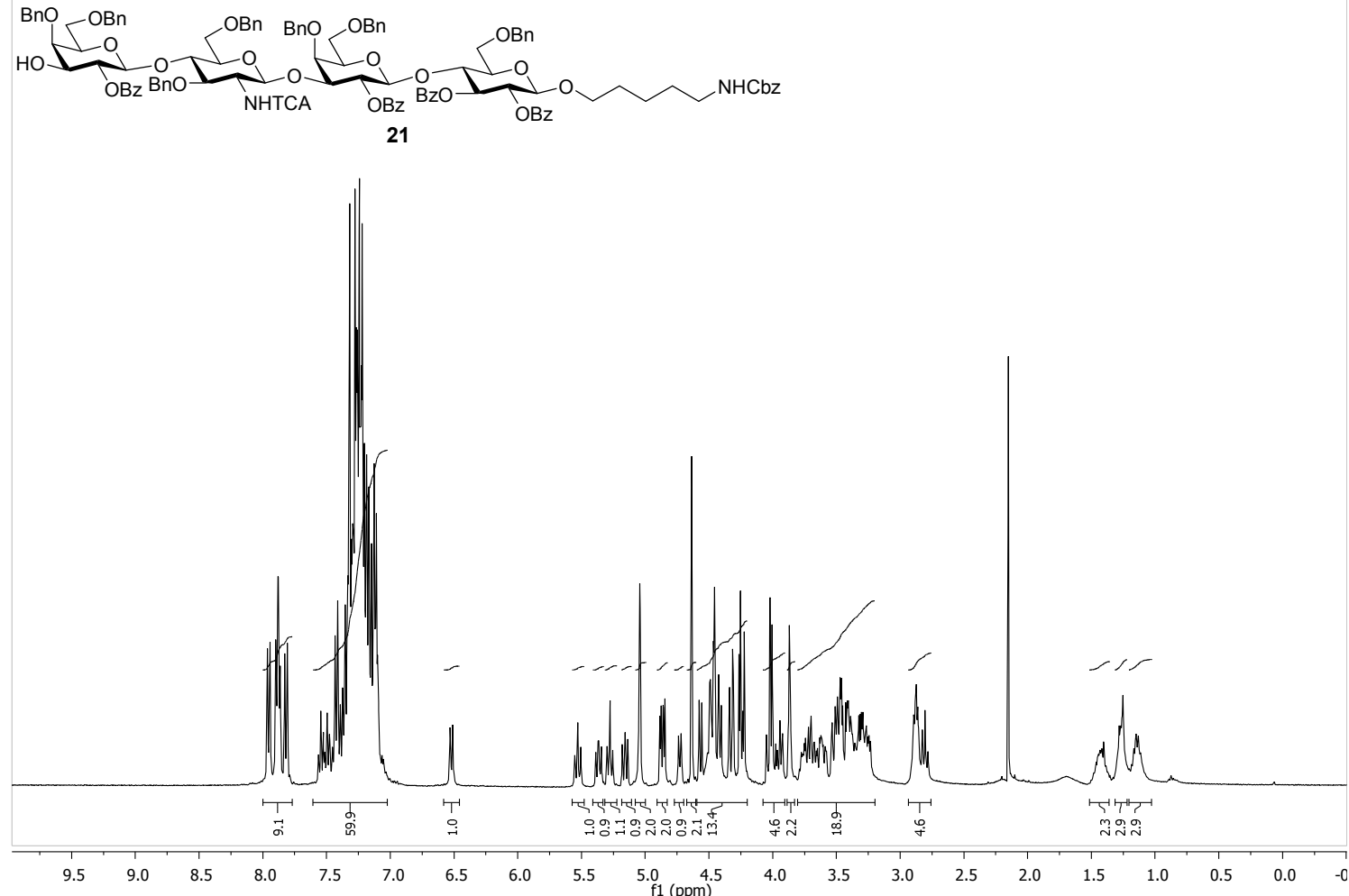

${ }^{13} \mathrm{C} \mathrm{NMR}, 150 \mathrm{MHz}, \mathrm{CDCl}_{3}$

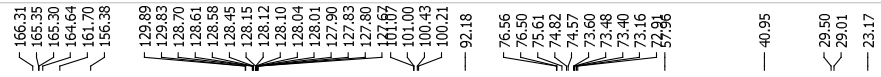

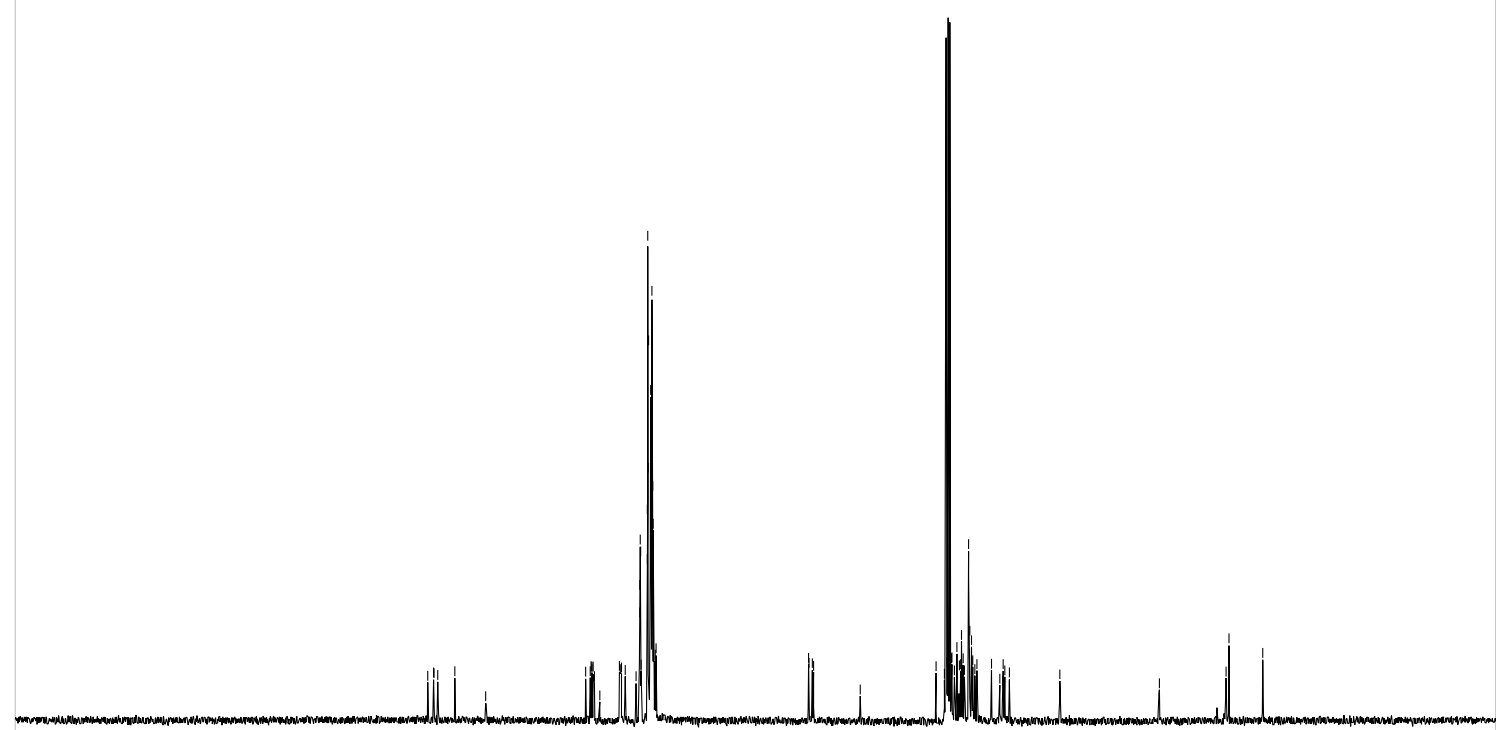

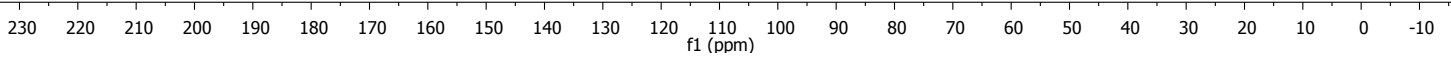


${ }^{1} \mathrm{H}-\mathrm{COSY} \mathrm{NMR}, 600 \mathrm{MHz}, \mathrm{CDCl}_{3}$

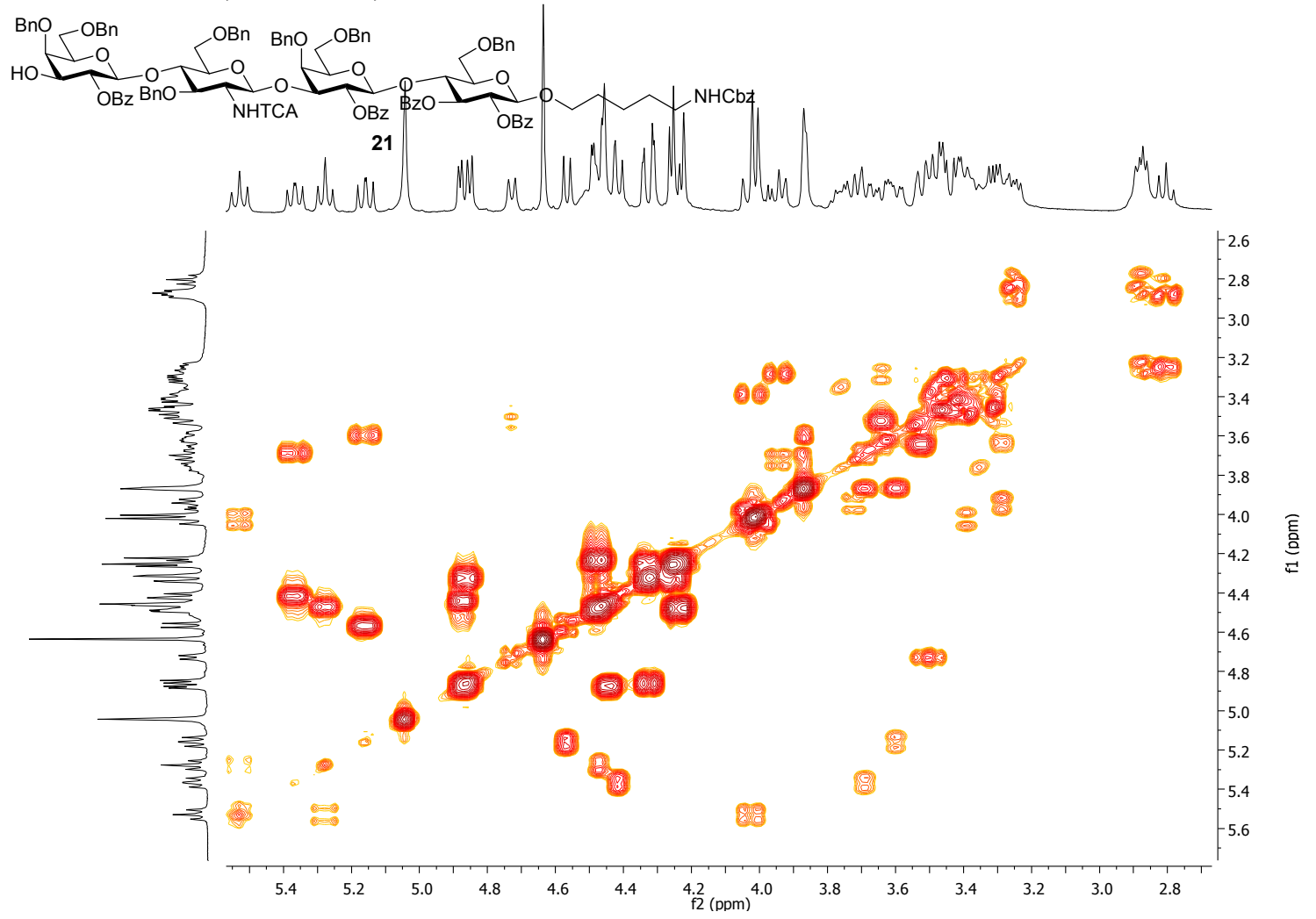

${ }^{1} \mathrm{H}^{-13} \mathrm{C}$-HSQC NMR, $600 \mathrm{MHz}, \mathrm{CDCl}_{3}$

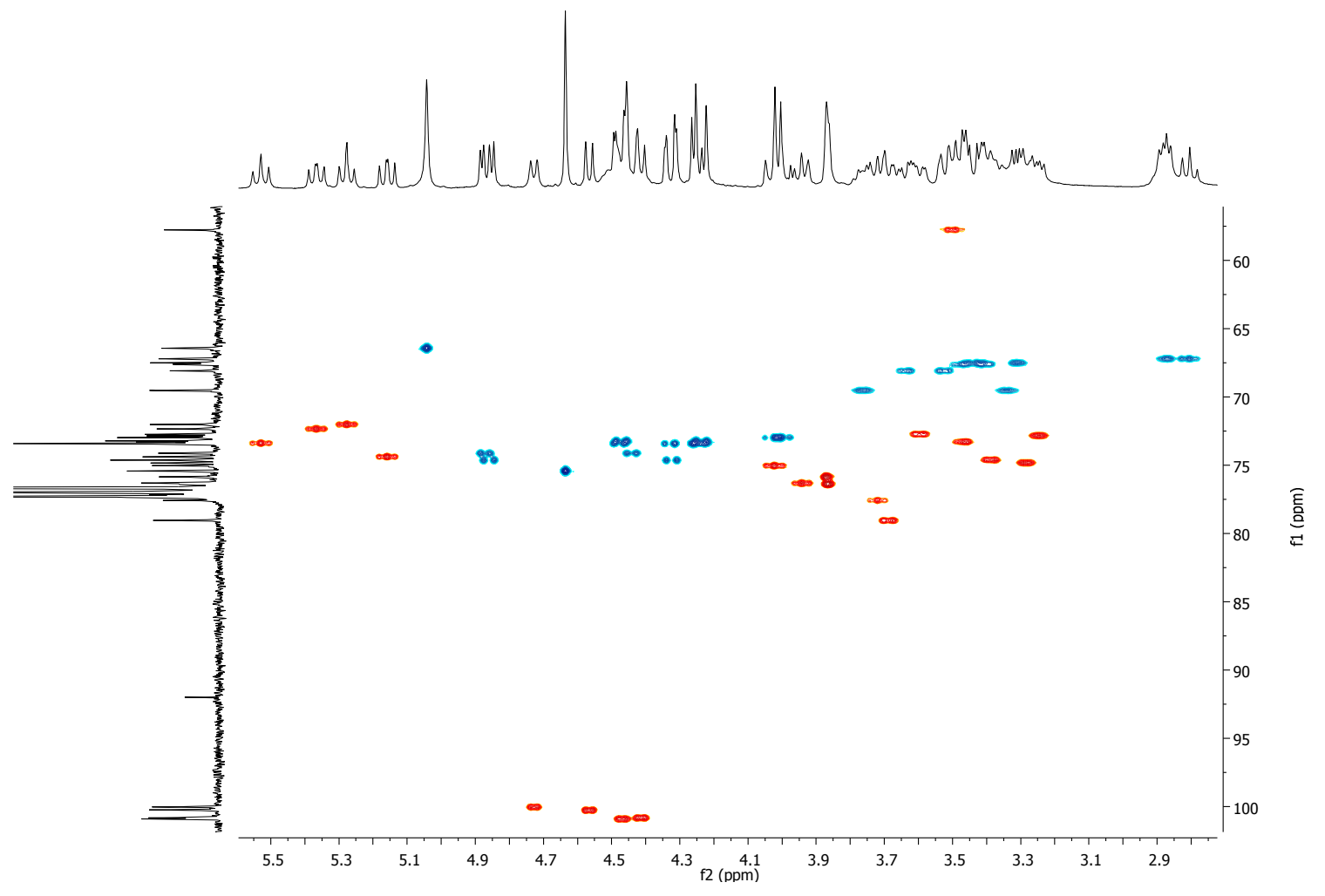


${ }^{1} \mathrm{H} \mathrm{NMR}, 600 \mathrm{MHz}, \mathrm{CDCl}_{3}$

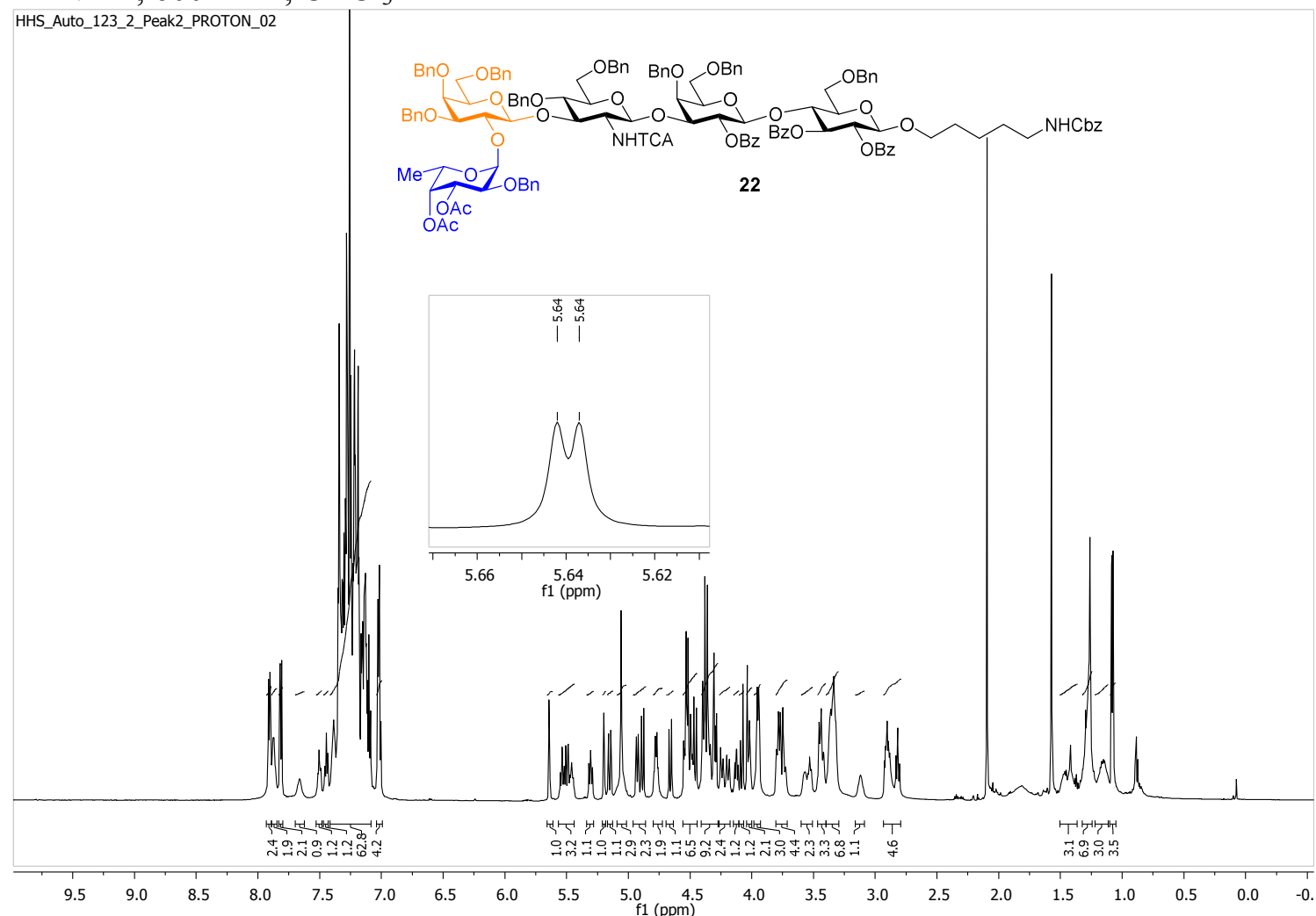

${ }^{13} \mathrm{C} \mathrm{NMR}, 150 \mathrm{MHz}, \mathrm{CDCl}_{3}$
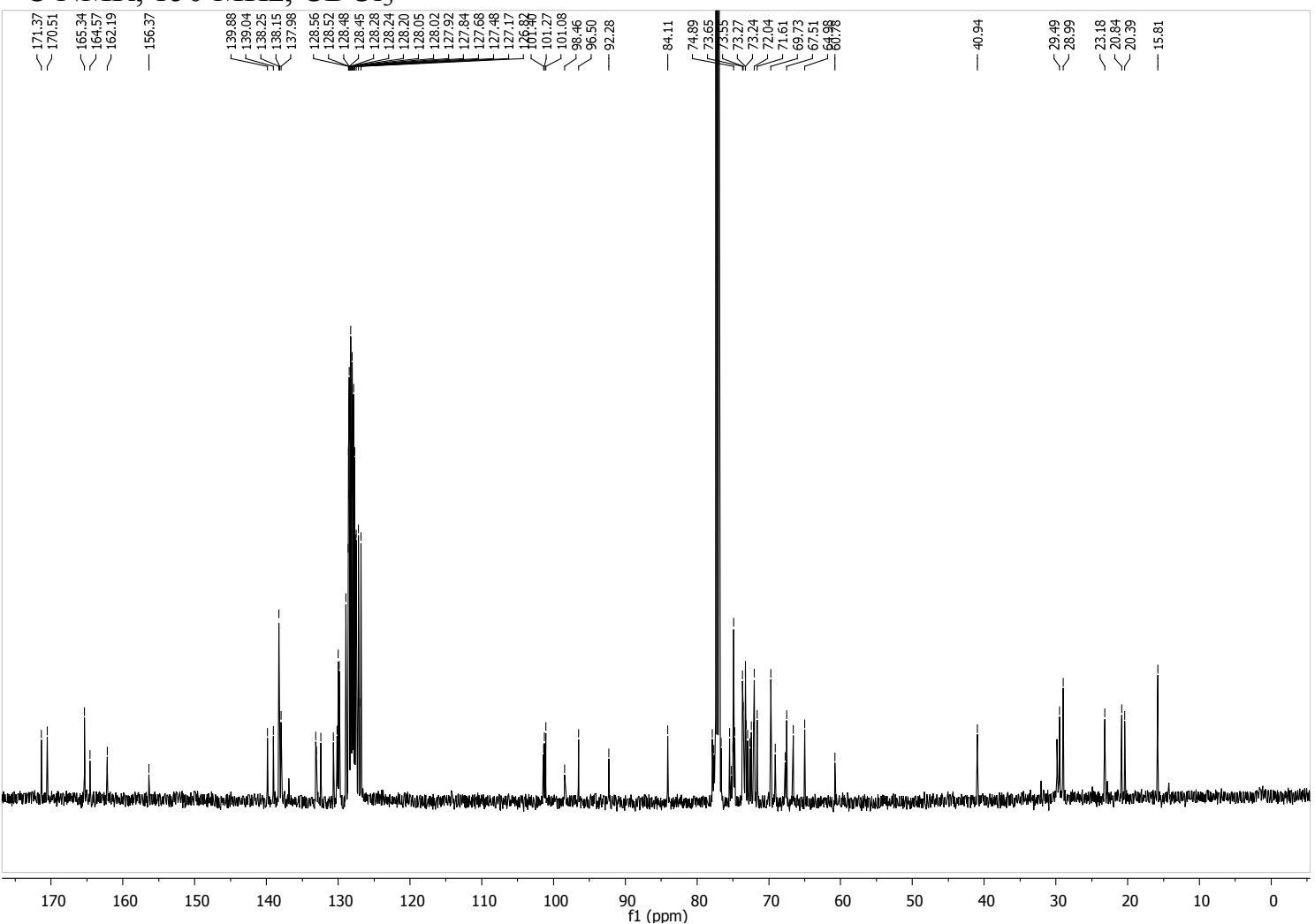
${ }^{1} \mathrm{H}-\mathrm{COSY} \mathrm{NMR}, 600 \mathrm{MHz}, \mathrm{CDCl}_{3}$

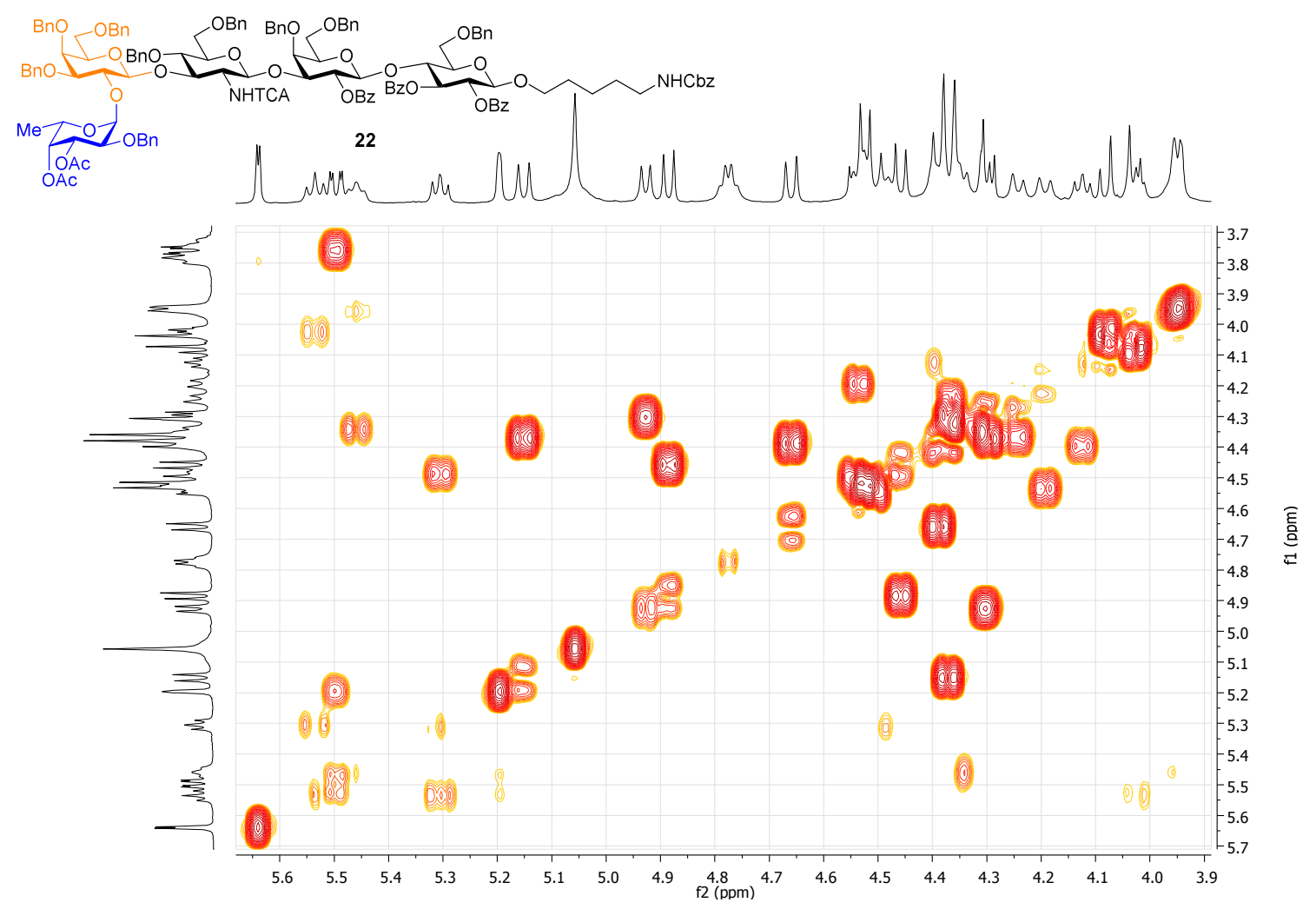

${ }^{1} \mathrm{H}_{-}{ }^{13} \mathrm{C}-\mathrm{HSQC} \mathrm{NMR}, 600 \mathrm{MHz}, \mathrm{CDCl}_{3}$

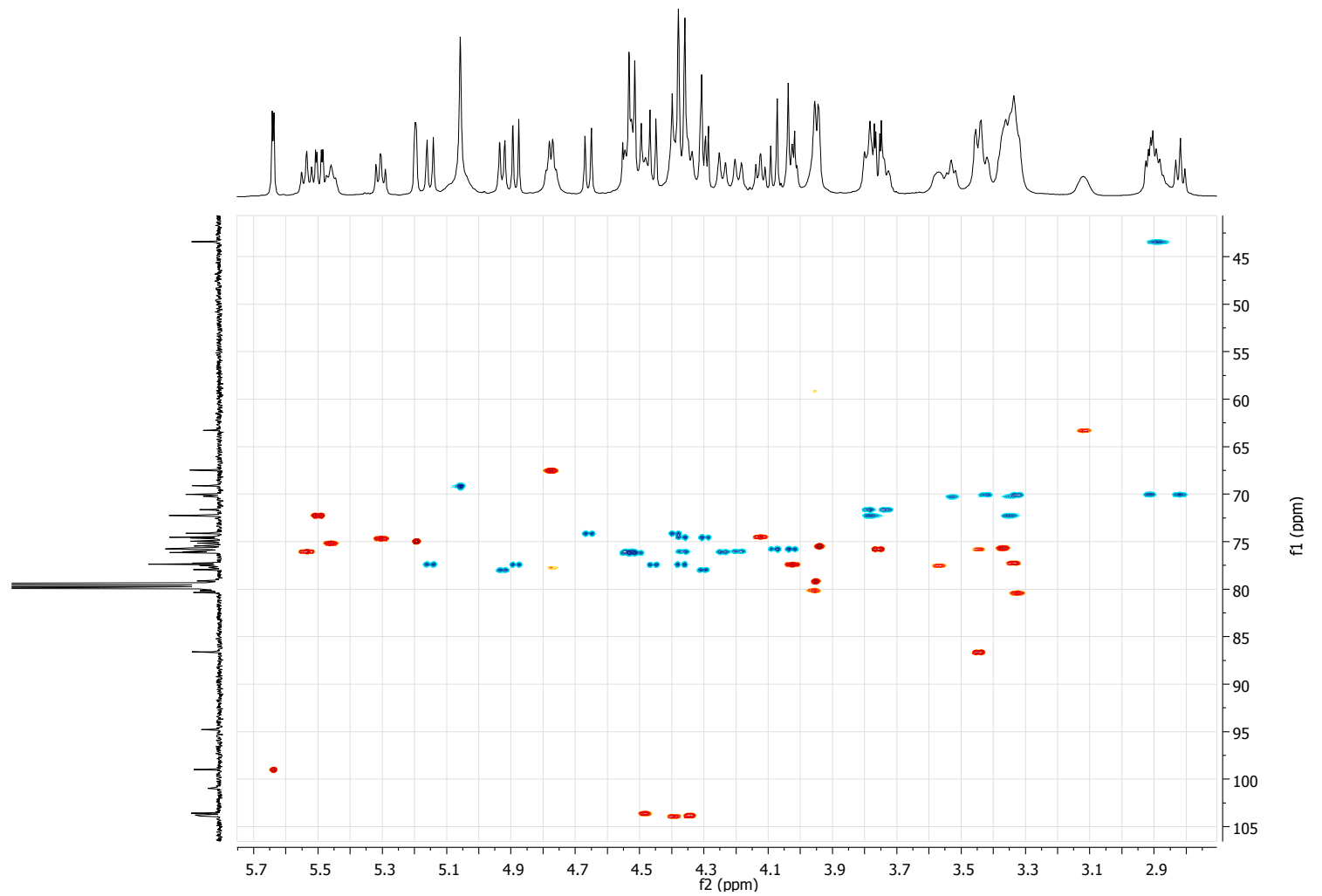


${ }^{1} \mathrm{H}-{ }^{13} \mathrm{C}$-coupled-HSQC NMR, $600 \mathrm{MHz}, \mathrm{CDCl}_{3}$
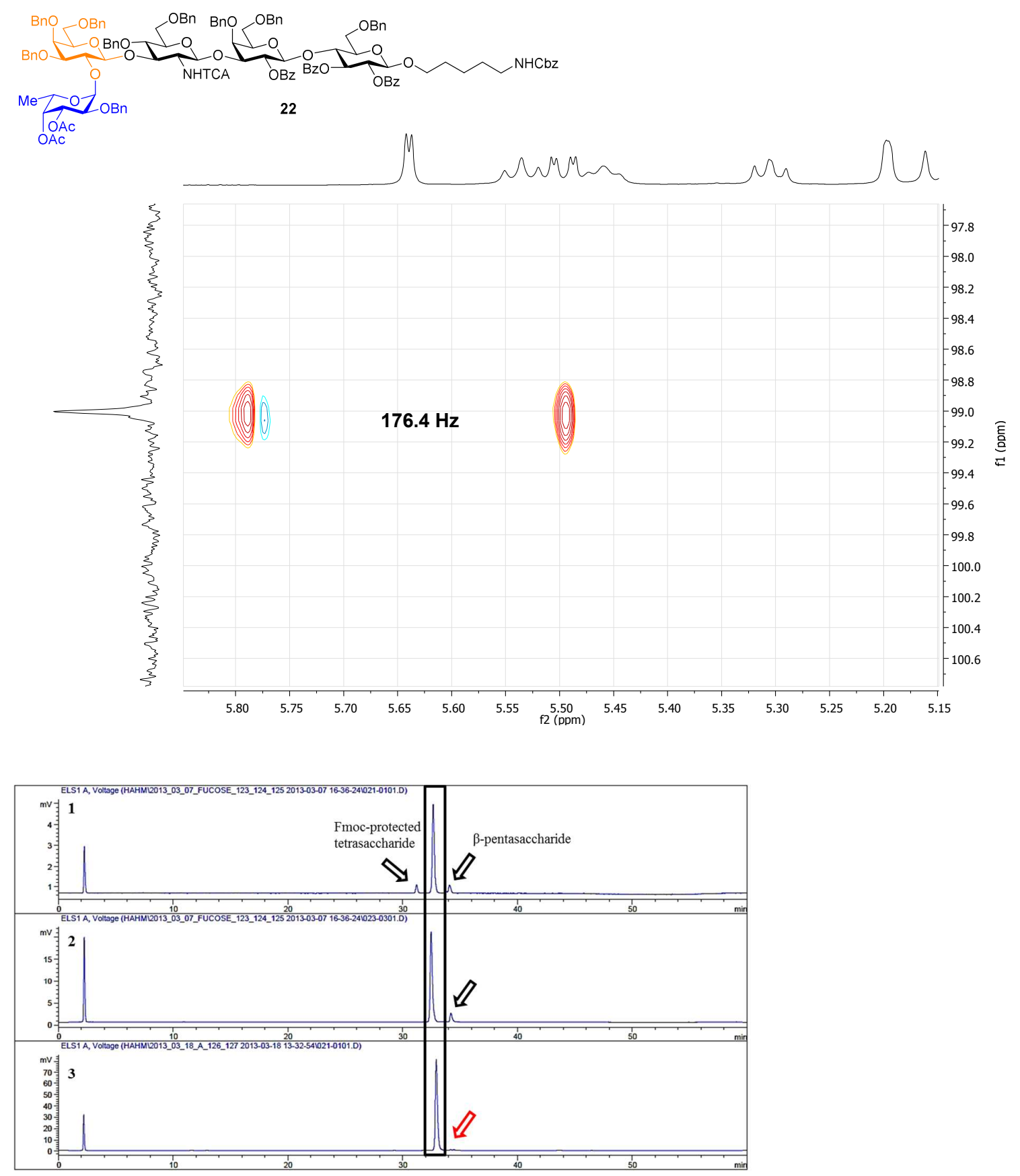

Figure S2. LC-MS of pentasaccharide 22. Condition: 17 dissolved in DCM and $\mathrm{Et}_{2} \mathrm{O}(\mathrm{v} / \mathrm{v}, 1 / 3)$ for entries 1 and 2. 17 dissolved in DCM for entry 3. 

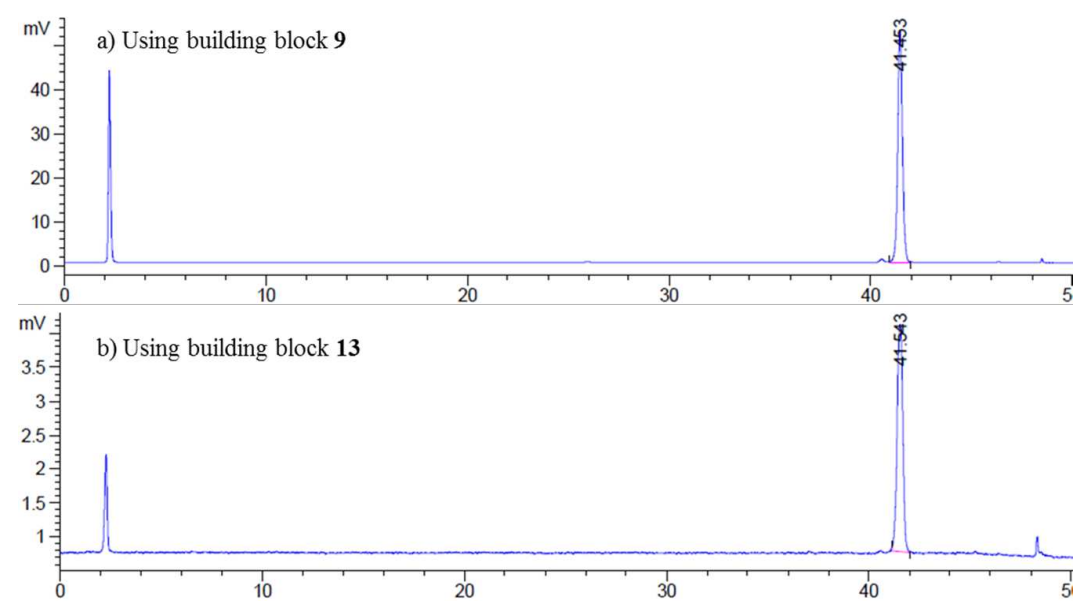

Figure S3. LC-MS of H-type II using fucose building block 17 and 18.

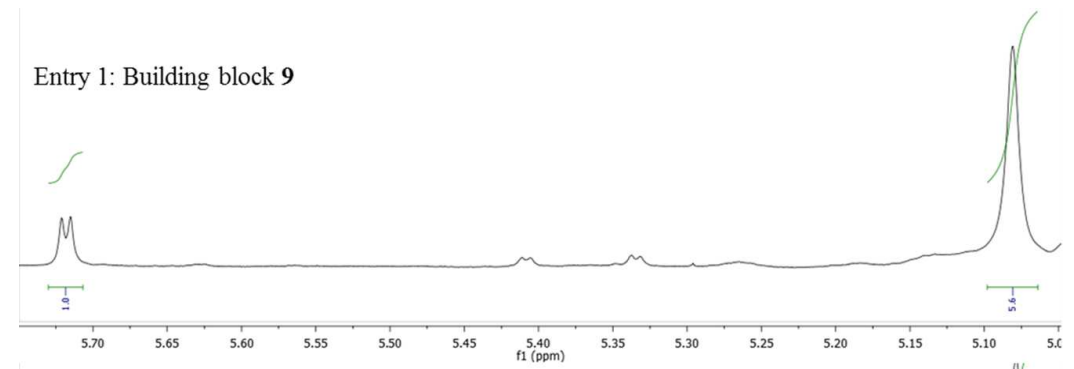

Entry 1: Building block 22

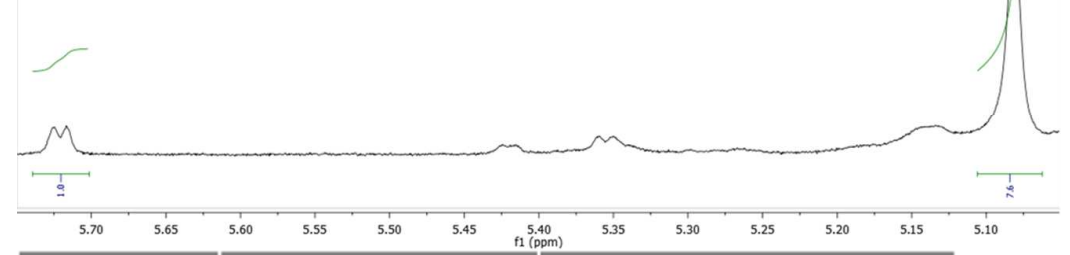

\begin{tabular}{|l|l|l|}
\hline Entry & Building Block & Ratio $(\mathbf{S 2 2} / \mathbf{S 2 3}=\alpha / \beta)$ \\
\hline 1 & $\mathbf{9}$ & $1: 1.8$ \\
\hline 2 & $\mathbf{2 2}$ & $1: 2.8$
\end{tabular}

Figure S5. Stereoselectivity of H-type II determined by ${ }^{1} \mathrm{H}$ NMR.

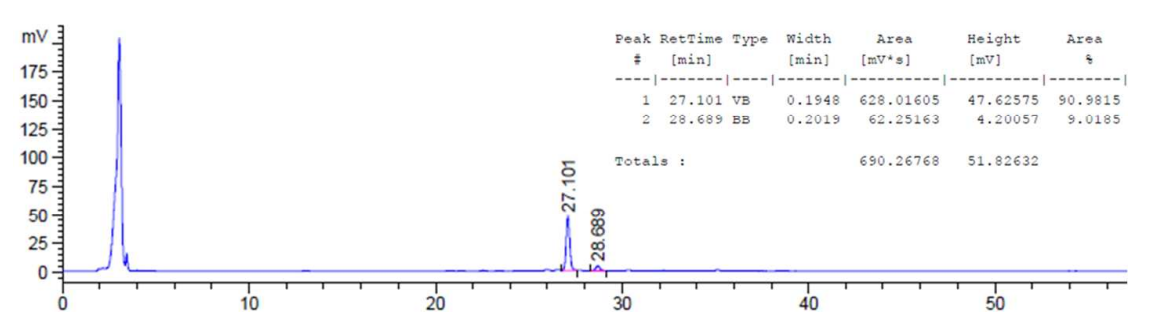

Figure S6. LC-MS of 23. 
${ }^{1} \mathrm{H} \mathrm{NMR}, 600 \mathrm{MHz}, \mathrm{CDCl}_{3}$

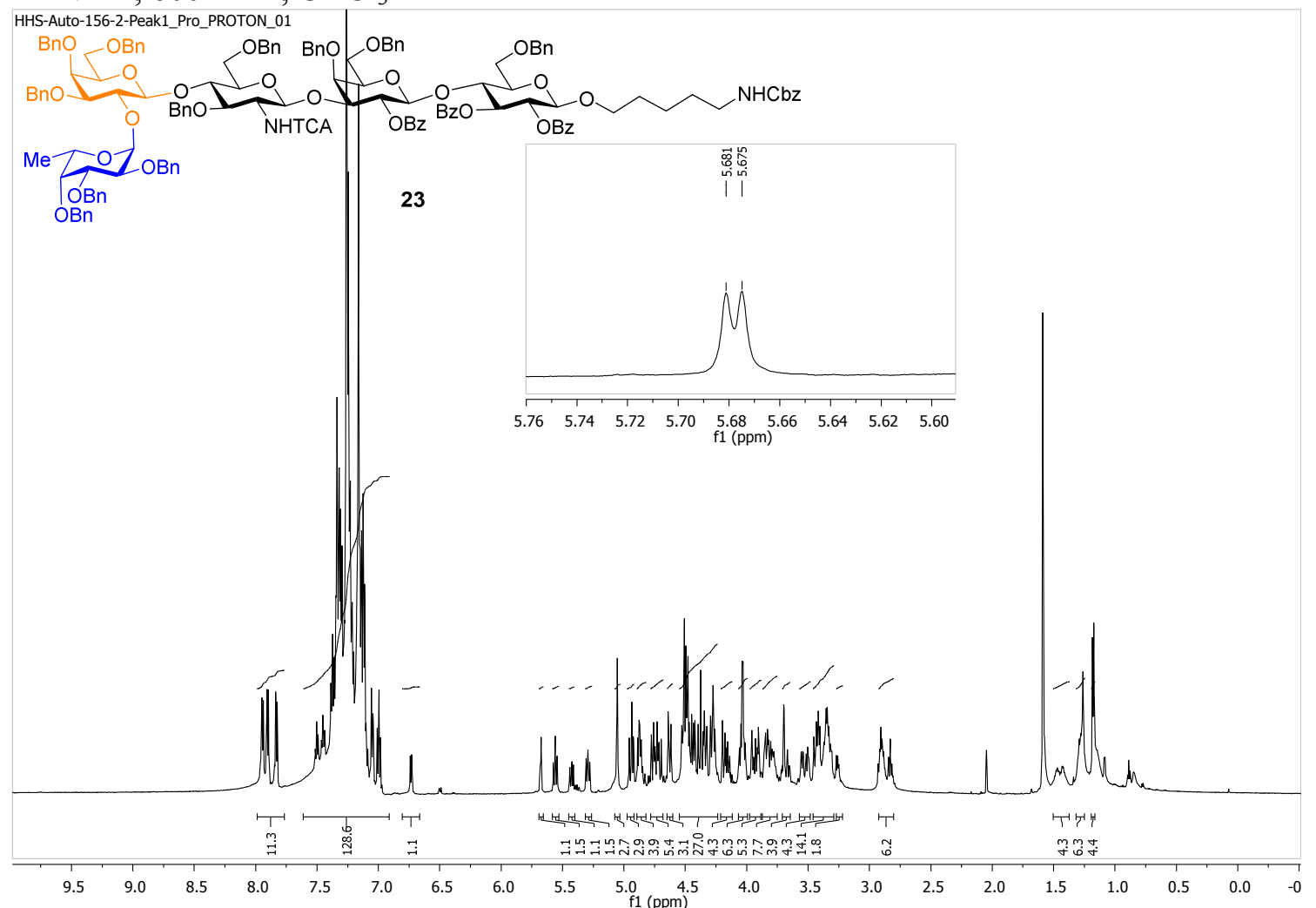

${ }^{13} \mathrm{C} \mathrm{NMR}, 150 \mathrm{MHz}, \mathrm{CDCl}_{3}$
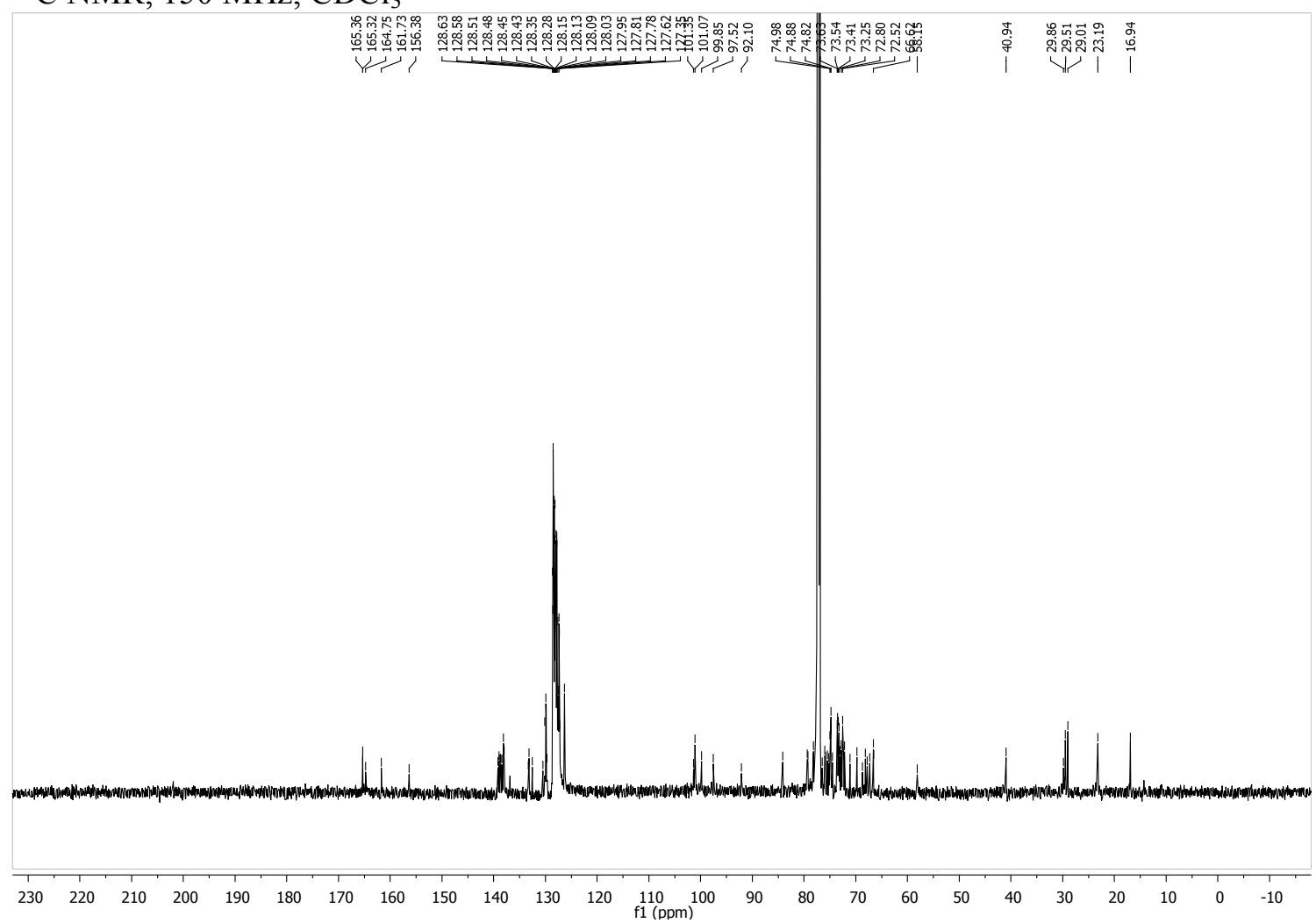
${ }^{1} \mathrm{H}-\mathrm{COSY} \mathrm{NMR}, 600 \mathrm{MHz}, \mathrm{CDCl}_{3}$

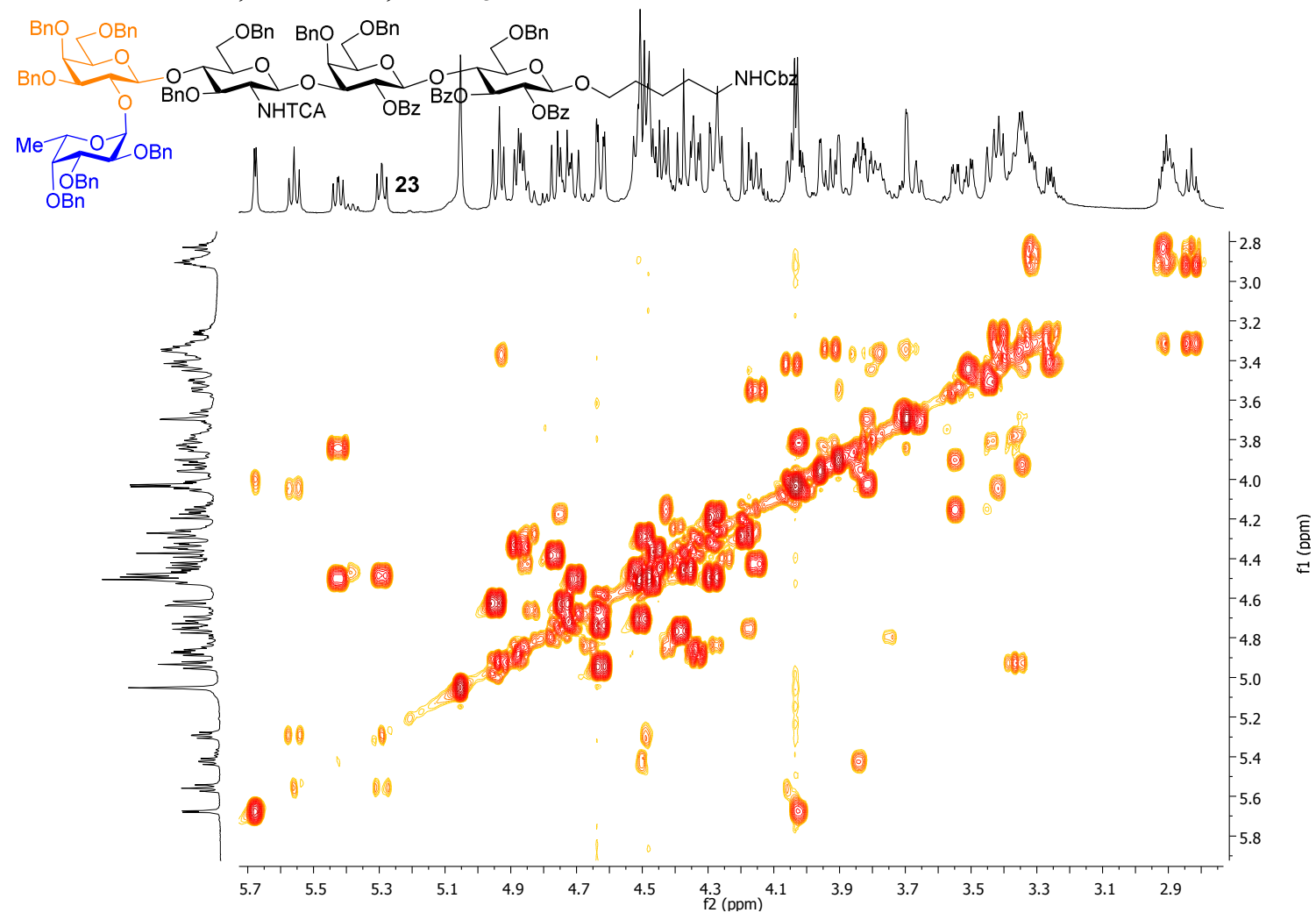

${ }^{1} \mathrm{H}^{13} \mathrm{C}-\mathrm{HSQC}$ NMR, $600 \mathrm{MHz}, \mathrm{CDCl}_{3}$

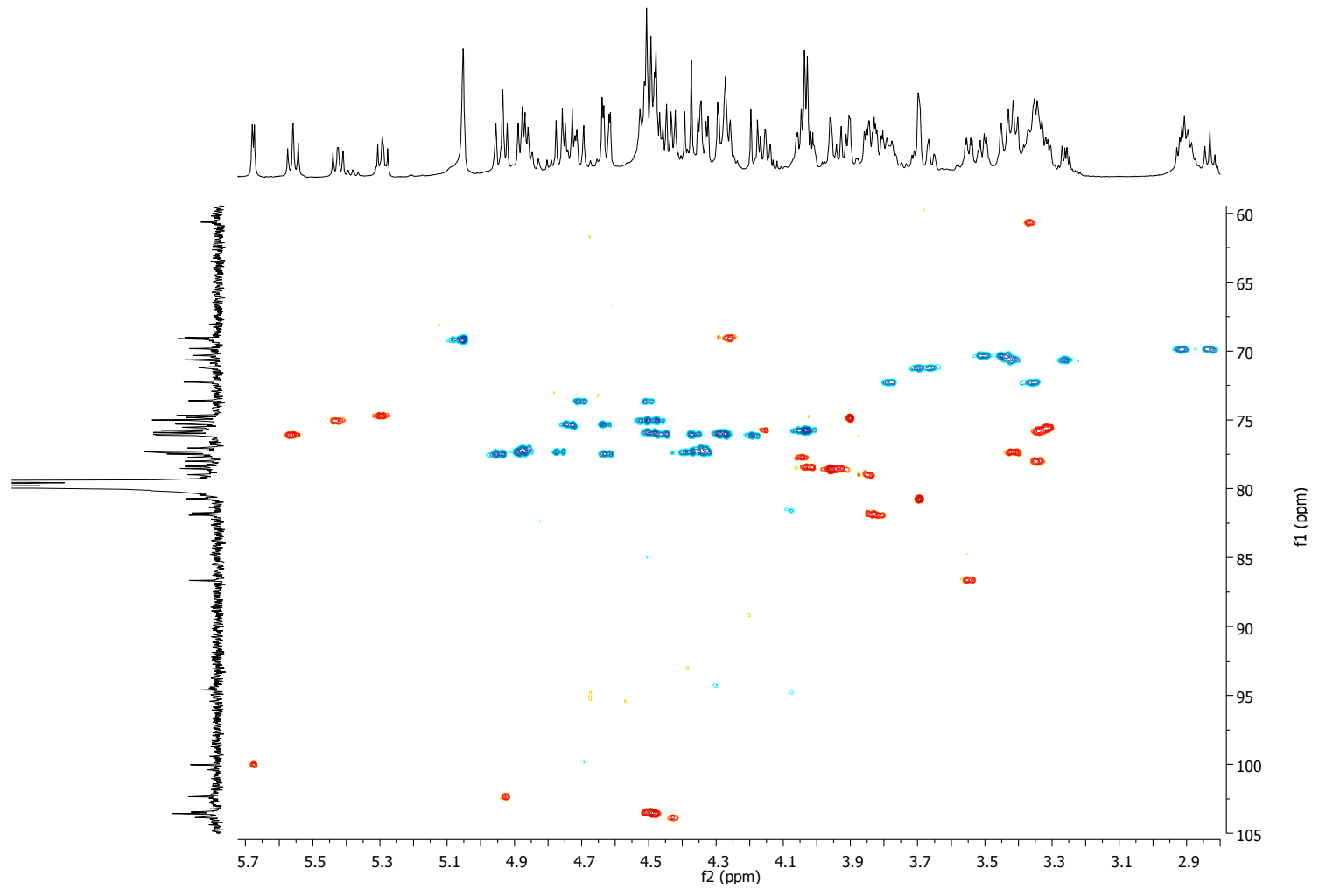


${ }^{1} \mathrm{H}-{ }^{13} \mathrm{C}$-coupled-HSQC NMR, $600 \mathrm{MHz}, \mathrm{CDCl}_{3}$

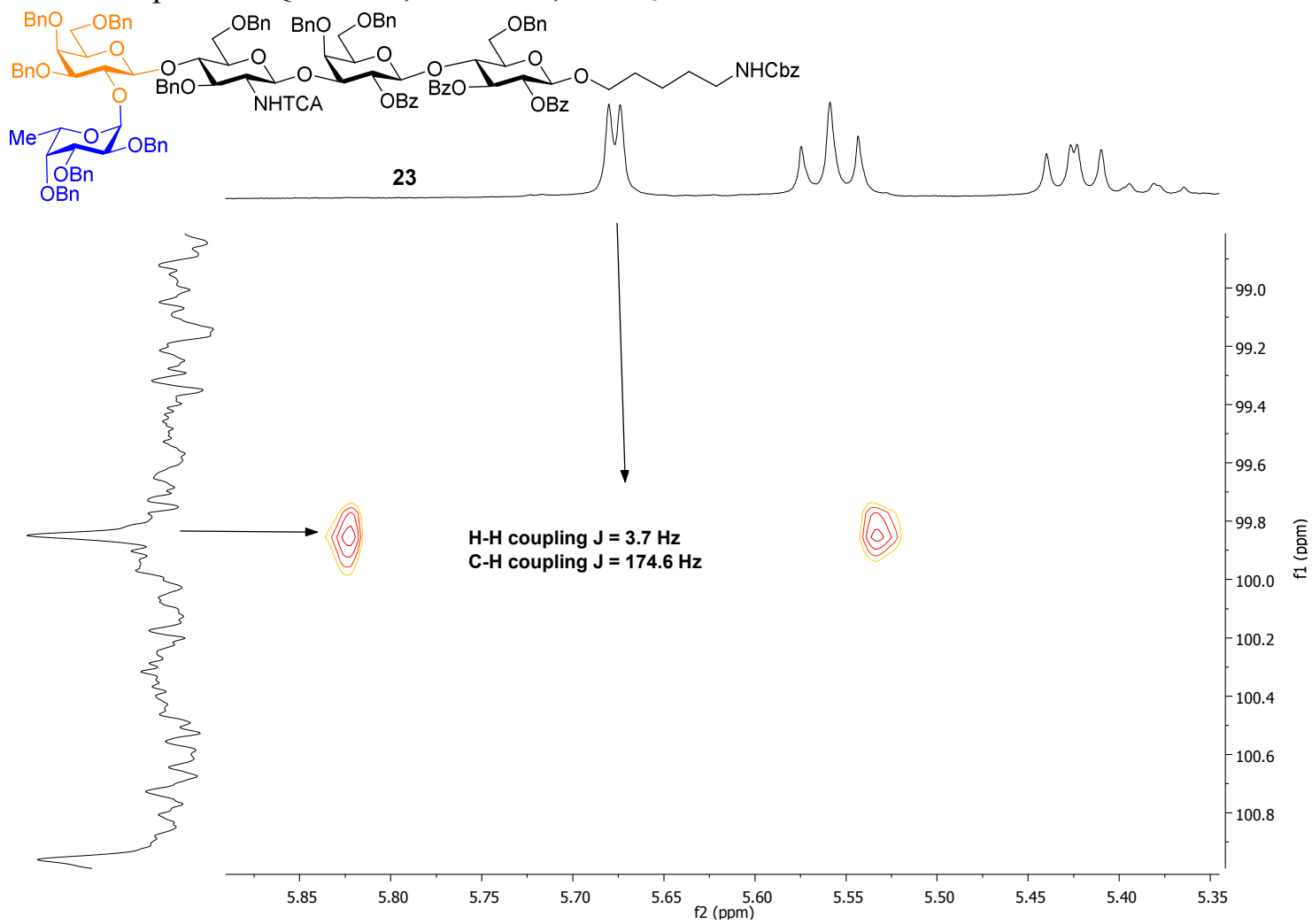




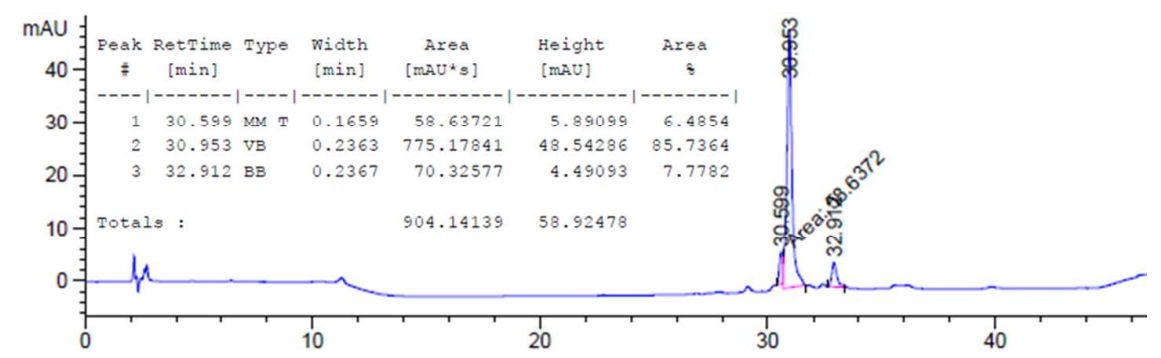

Figure S7. LC-MS of 28.

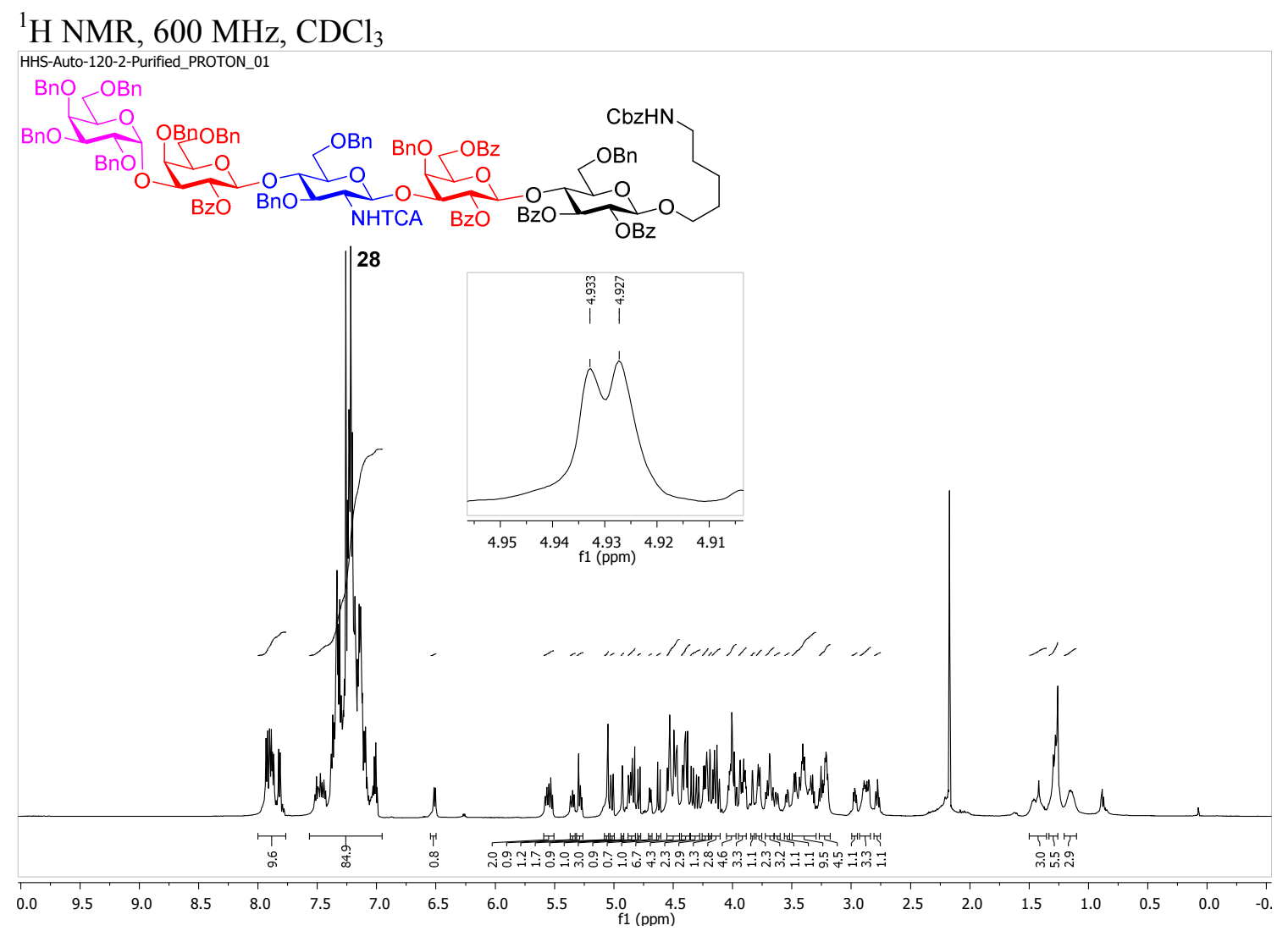


${ }^{13} \mathrm{C} \mathrm{NMR,}, 100 \mathrm{MHz}, \mathrm{CDCl}_{3}$

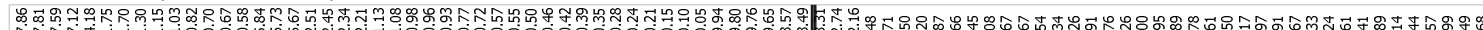

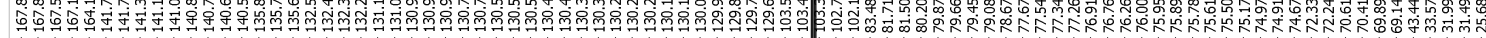
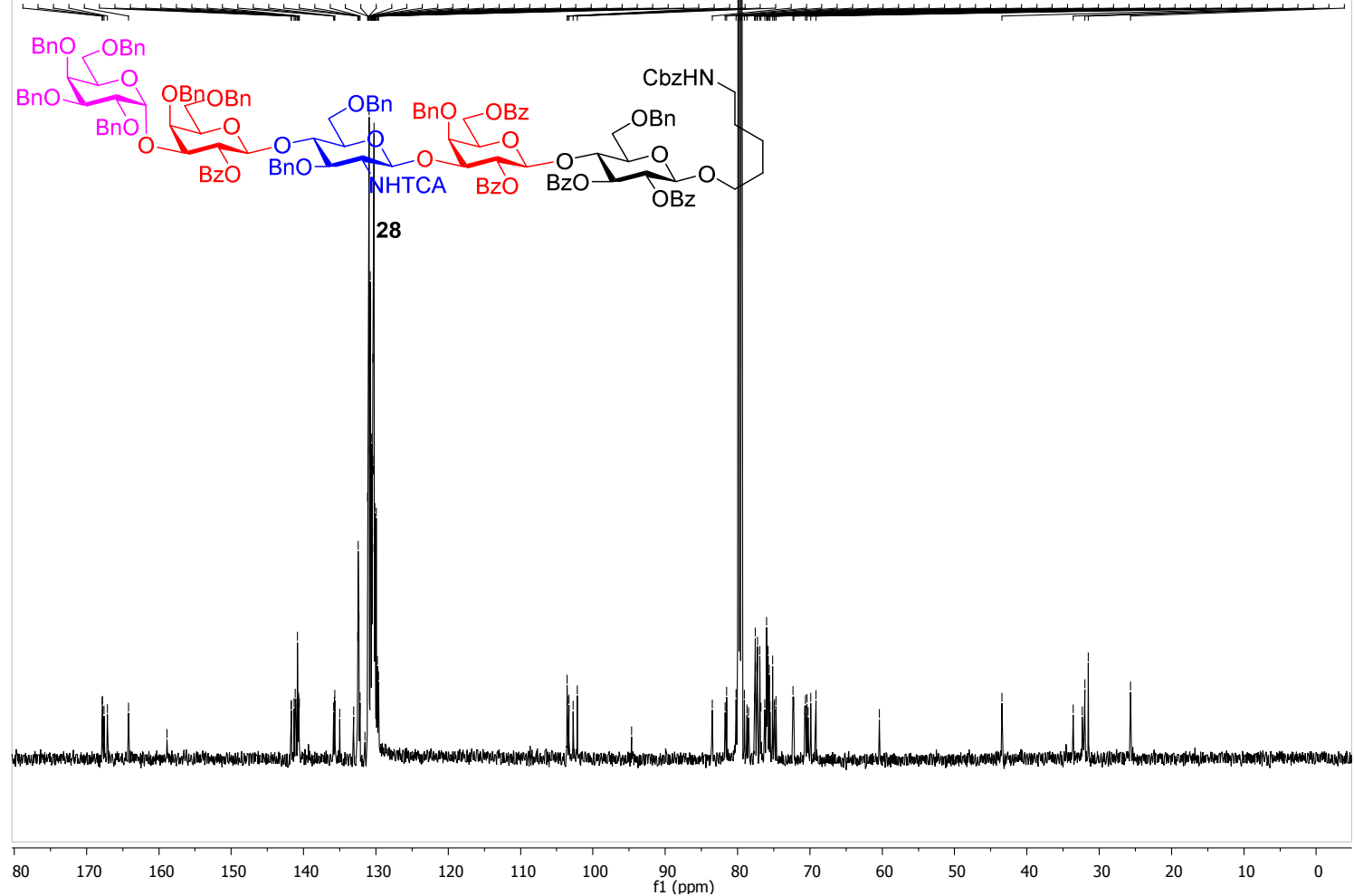

${ }^{1} \mathrm{H}^{13} \mathrm{C}$-coupled-HSQC NMR, $600 \mathrm{MHz}, \mathrm{CDCl}_{3}$

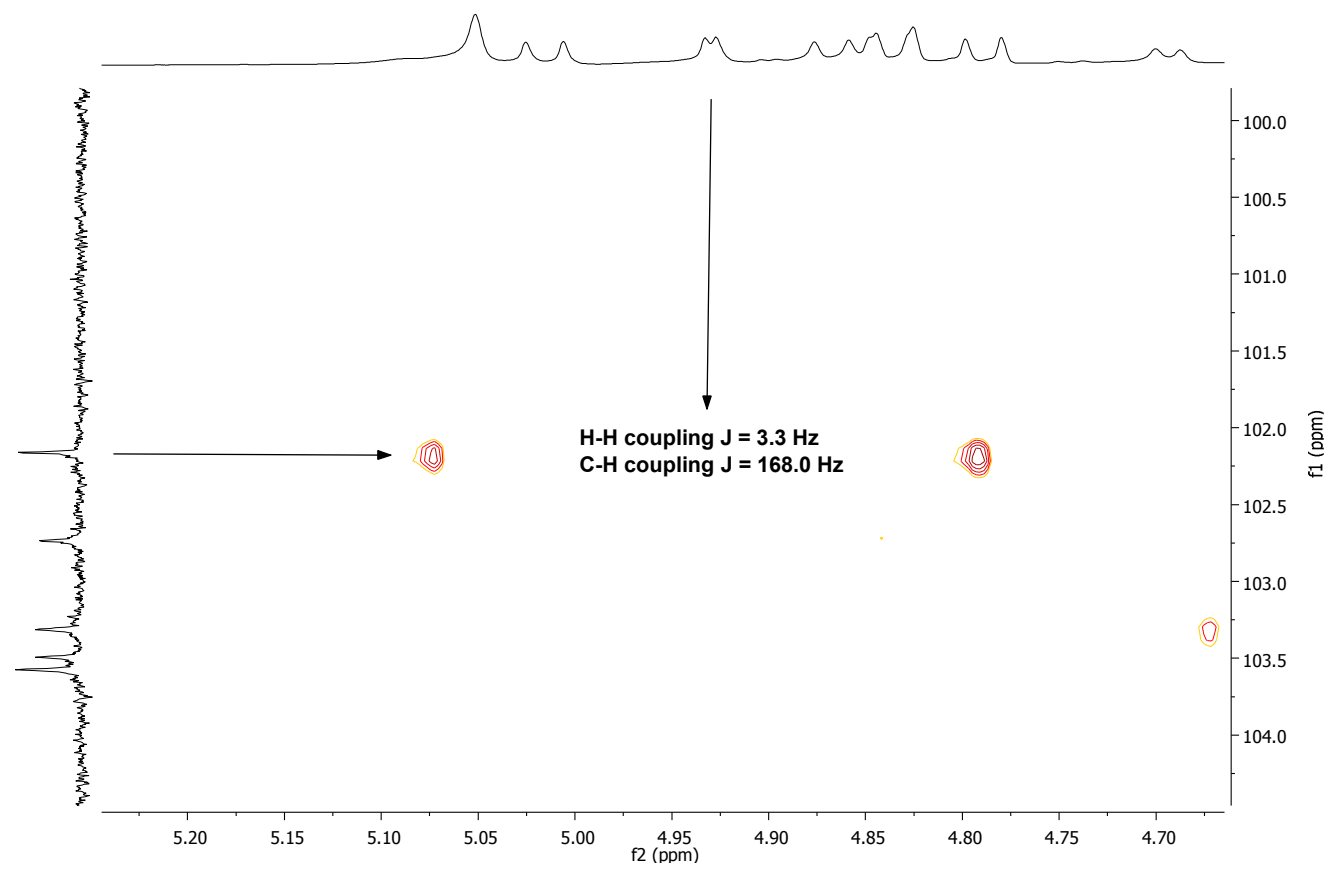




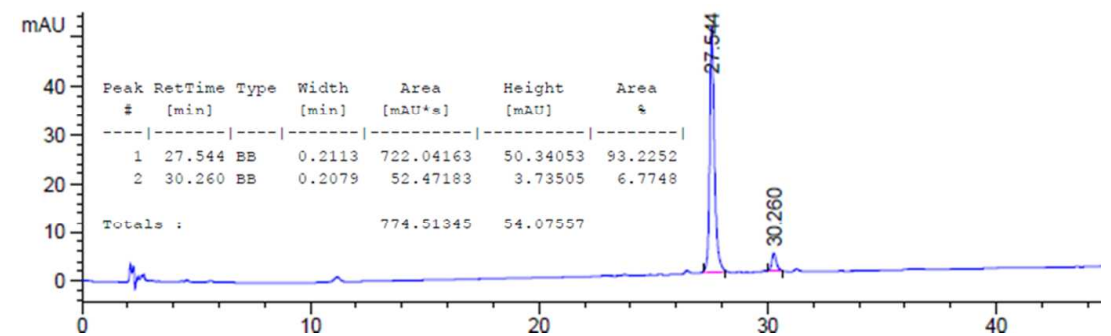

Figure S8. LC-MS of 29.

${ }^{1} \mathrm{H} \mathrm{NMR}, 600 \mathrm{MHz}, \mathrm{CDCl}_{3}$ HHS-Auto-115-2-Peak1_PROTON_13Mar13_01

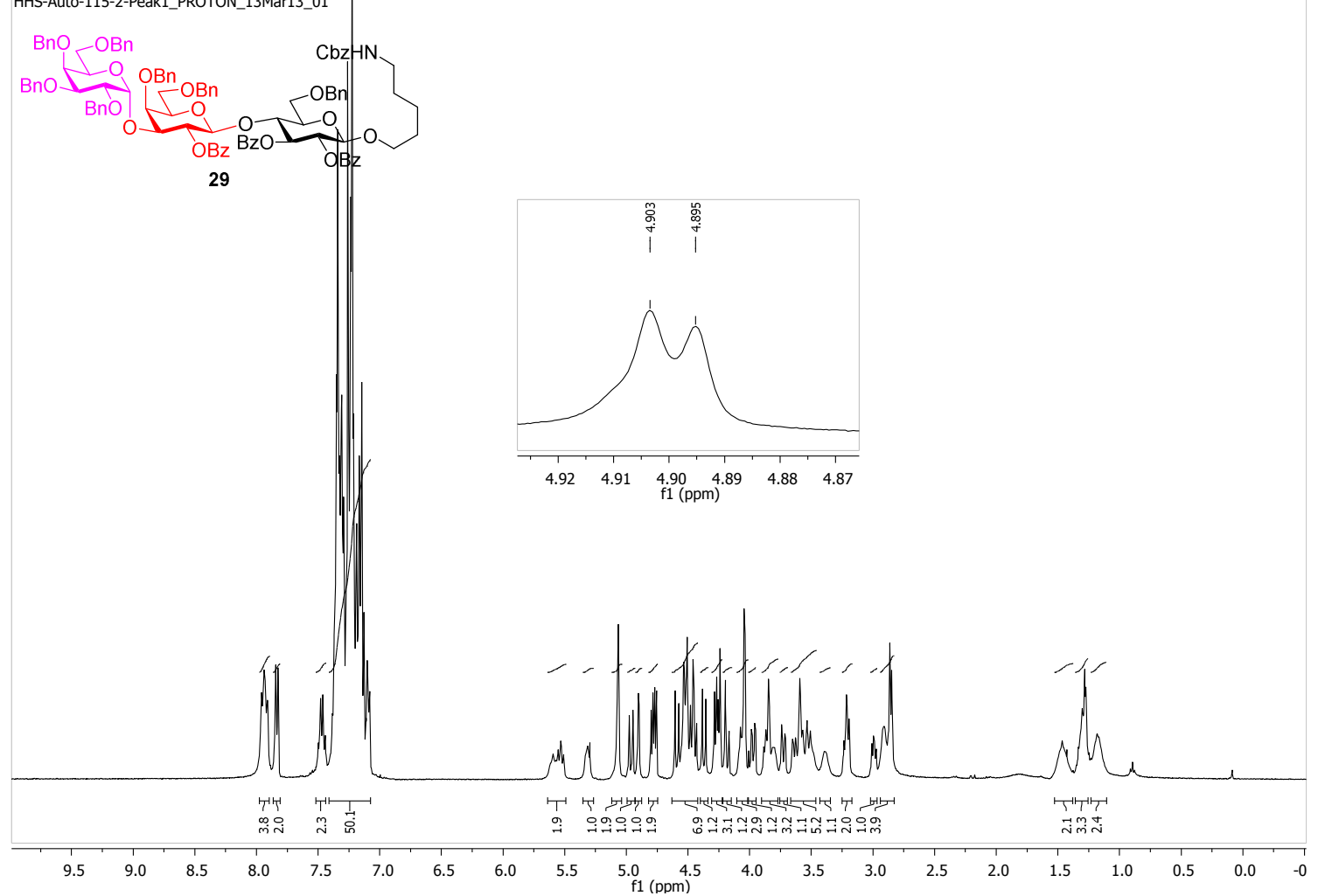


${ }^{13} \mathrm{C} \mathrm{NMR}, 150 \mathrm{MHz}, \mathrm{CDCl}_{3}$
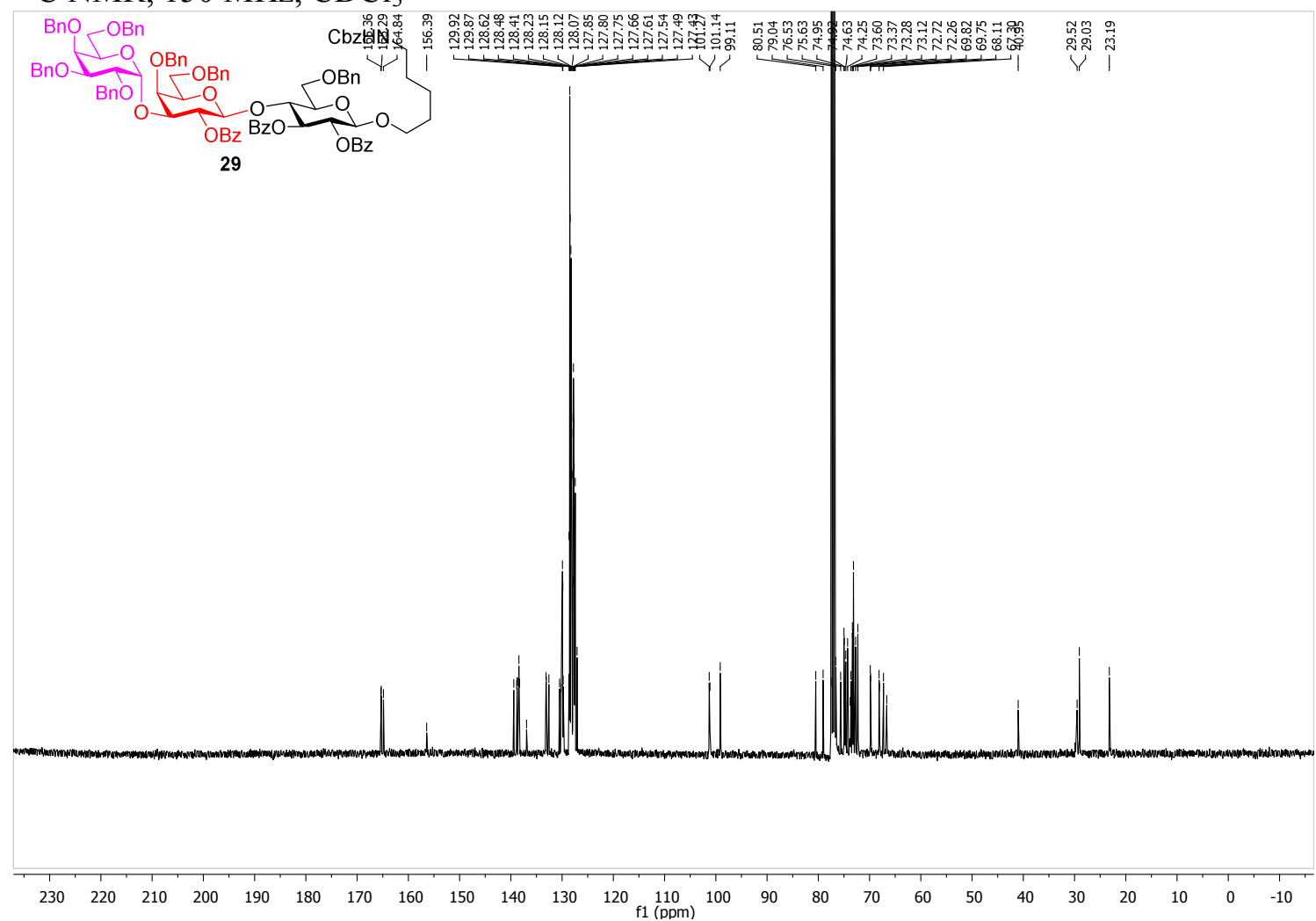

${ }^{1} \mathrm{H}-\mathrm{COSY} \mathrm{NMR}, 600 \mathrm{MHz}, \mathrm{CDCl}_{3}$

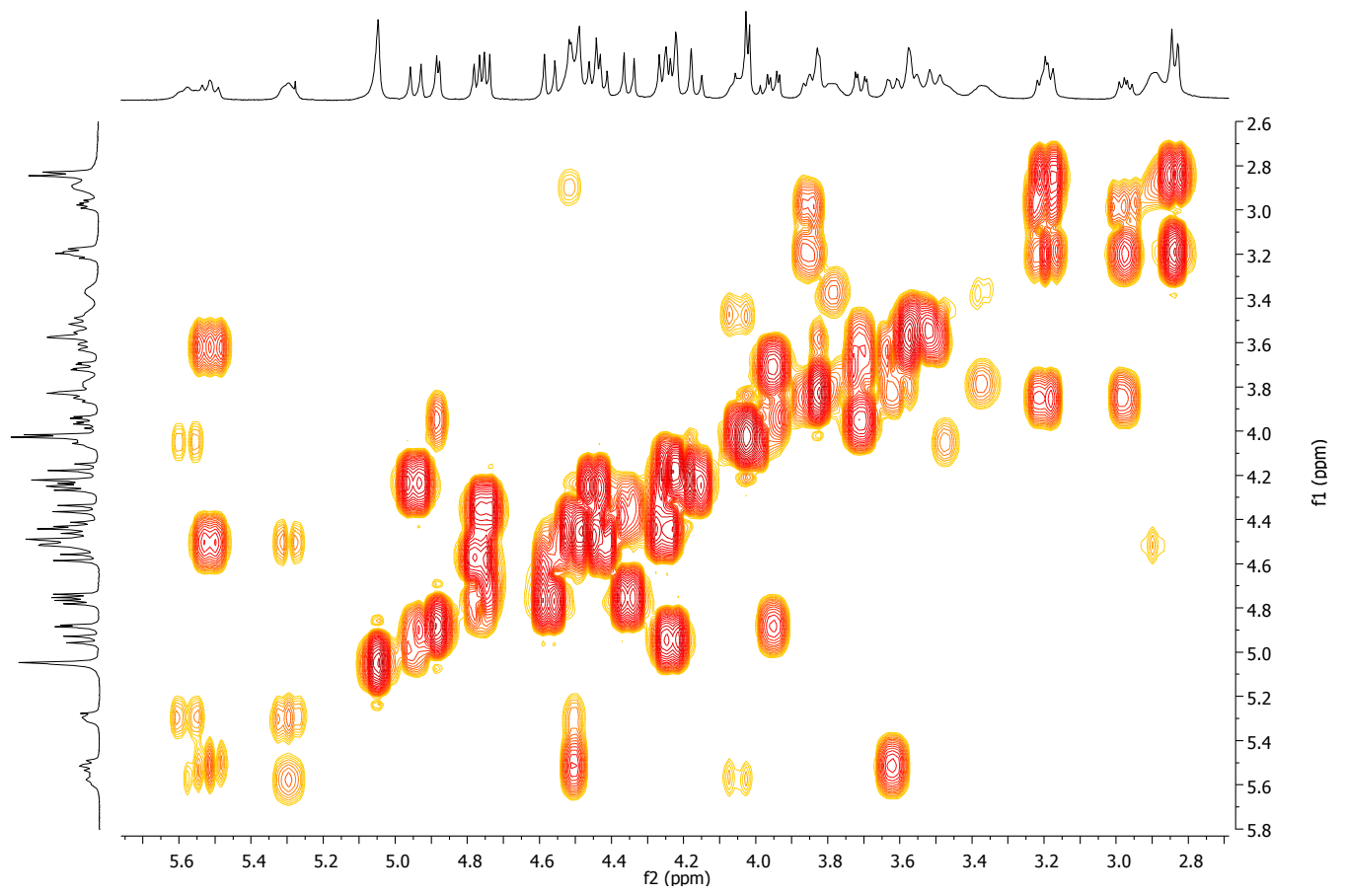


${ }^{1} \mathrm{H}^{13} \mathrm{C}-\mathrm{HSQC} \mathrm{NMR}, 600 \mathrm{MHz}, \mathrm{CDCl}_{3}$

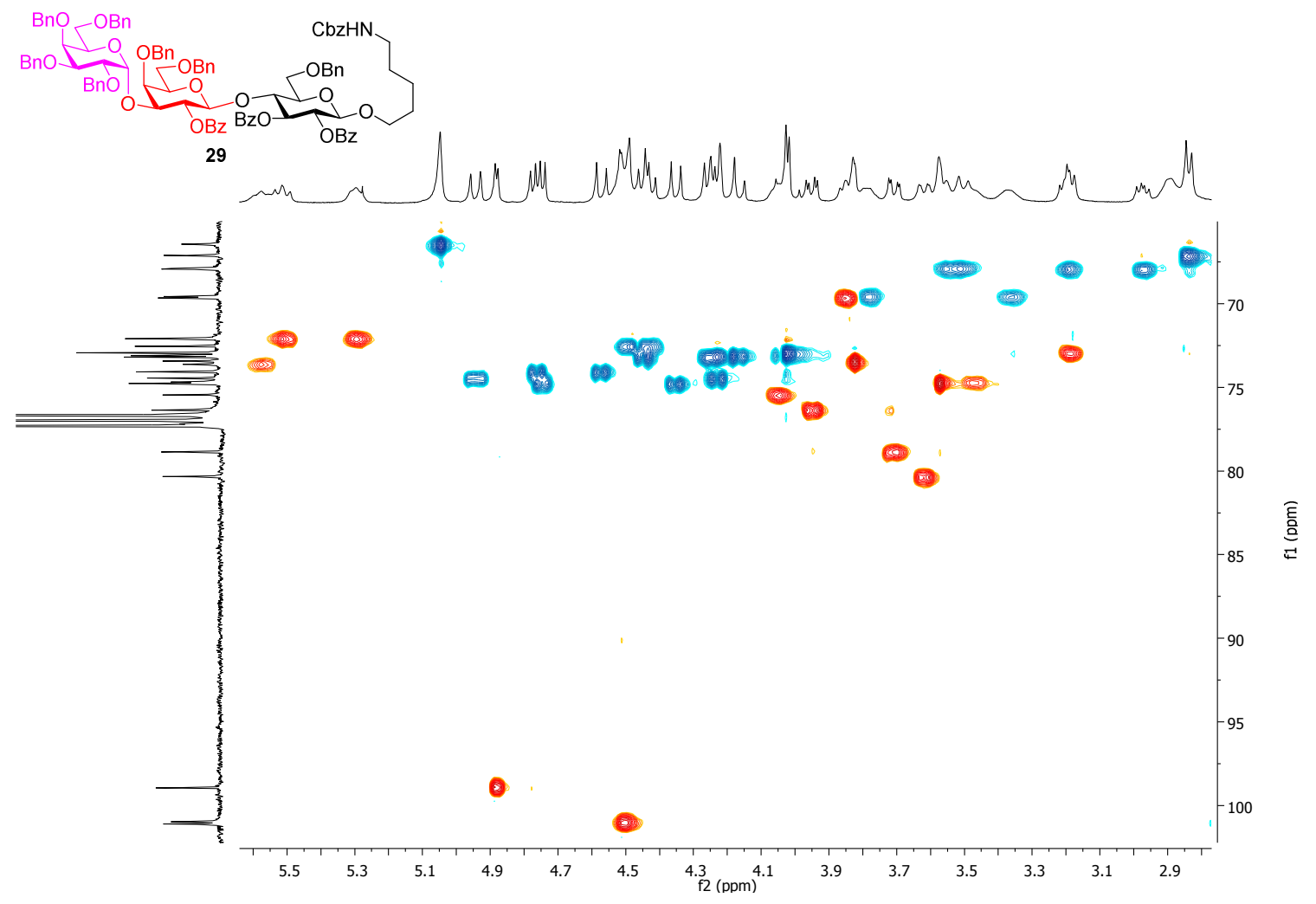

${ }^{1} \mathrm{H}-{ }^{13} \mathrm{C}$-coupled-HSQC NMR, $600 \mathrm{MHz}, \mathrm{CDCl}_{3}$

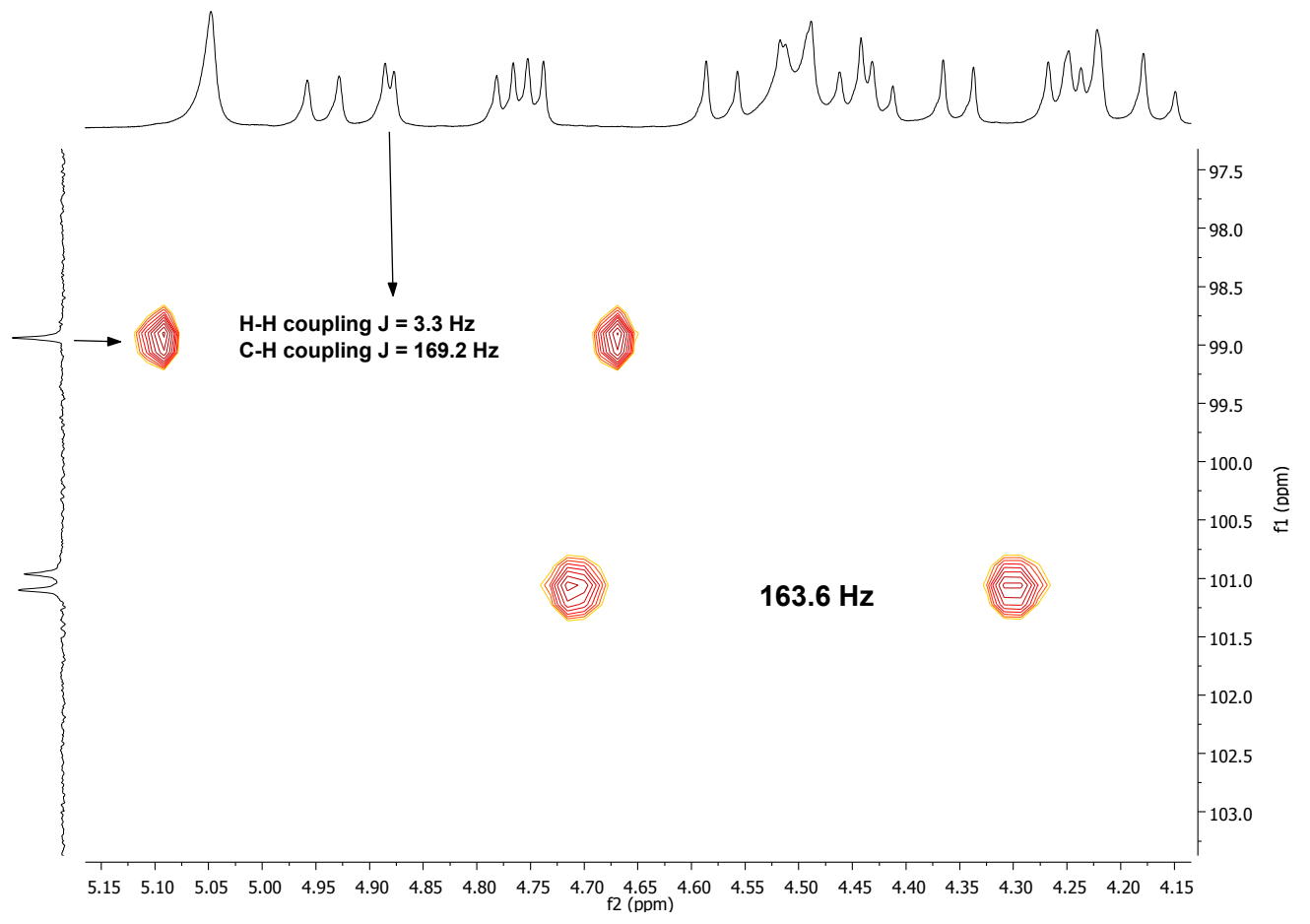




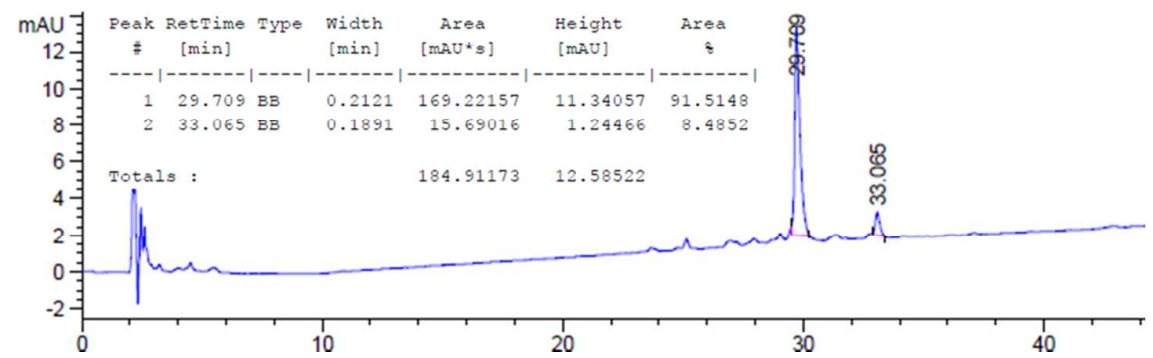

Figure S9. LC-MS of 30.

${ }^{1} \mathrm{H} \mathrm{NMR}, 600 \mathrm{MHz}, \mathrm{CDCl}_{3}$

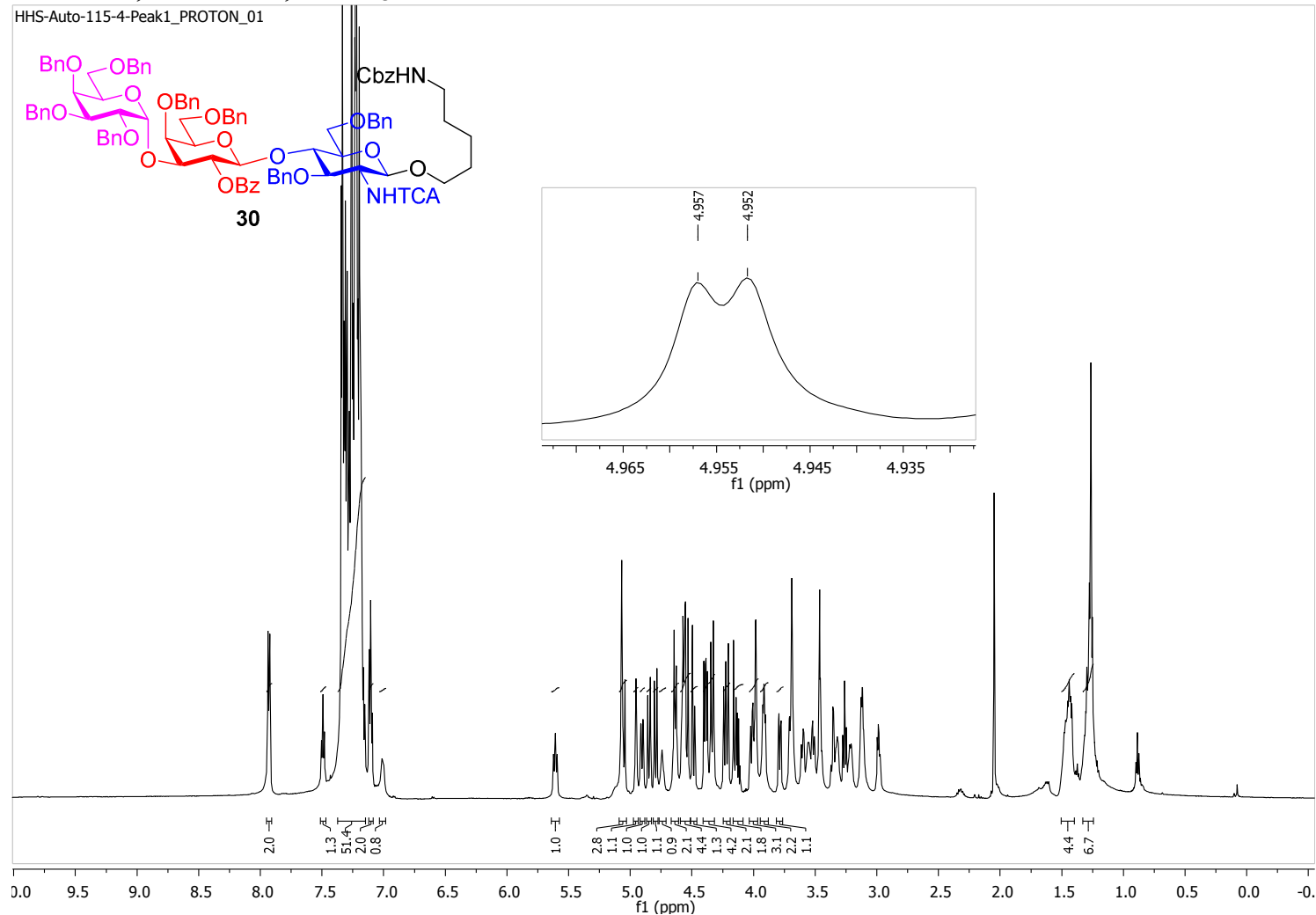



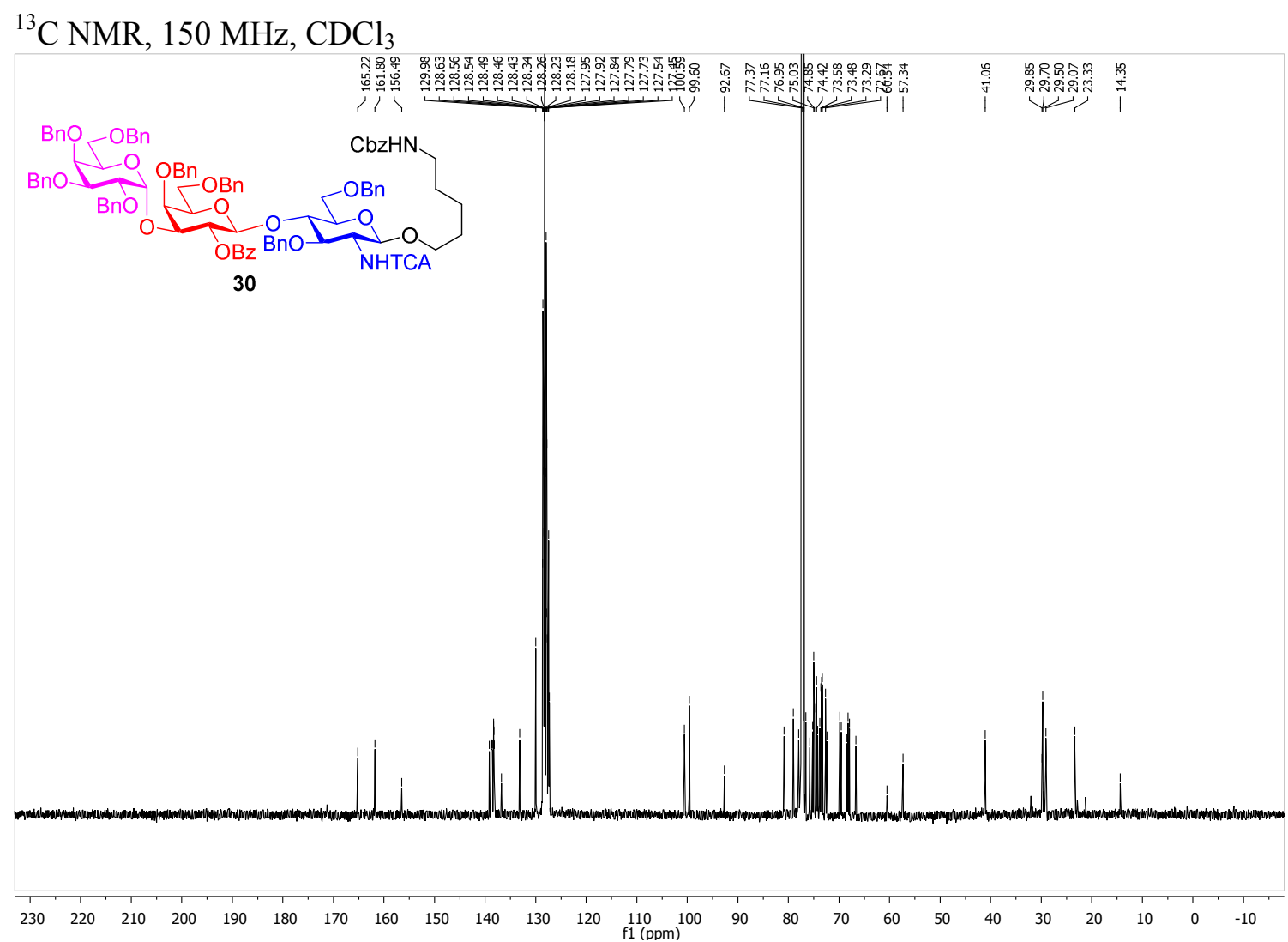

${ }^{1} \mathrm{H}-\mathrm{COSY} \mathrm{NMR}, 600 \mathrm{MHz}, \mathrm{CDCl}_{3}$

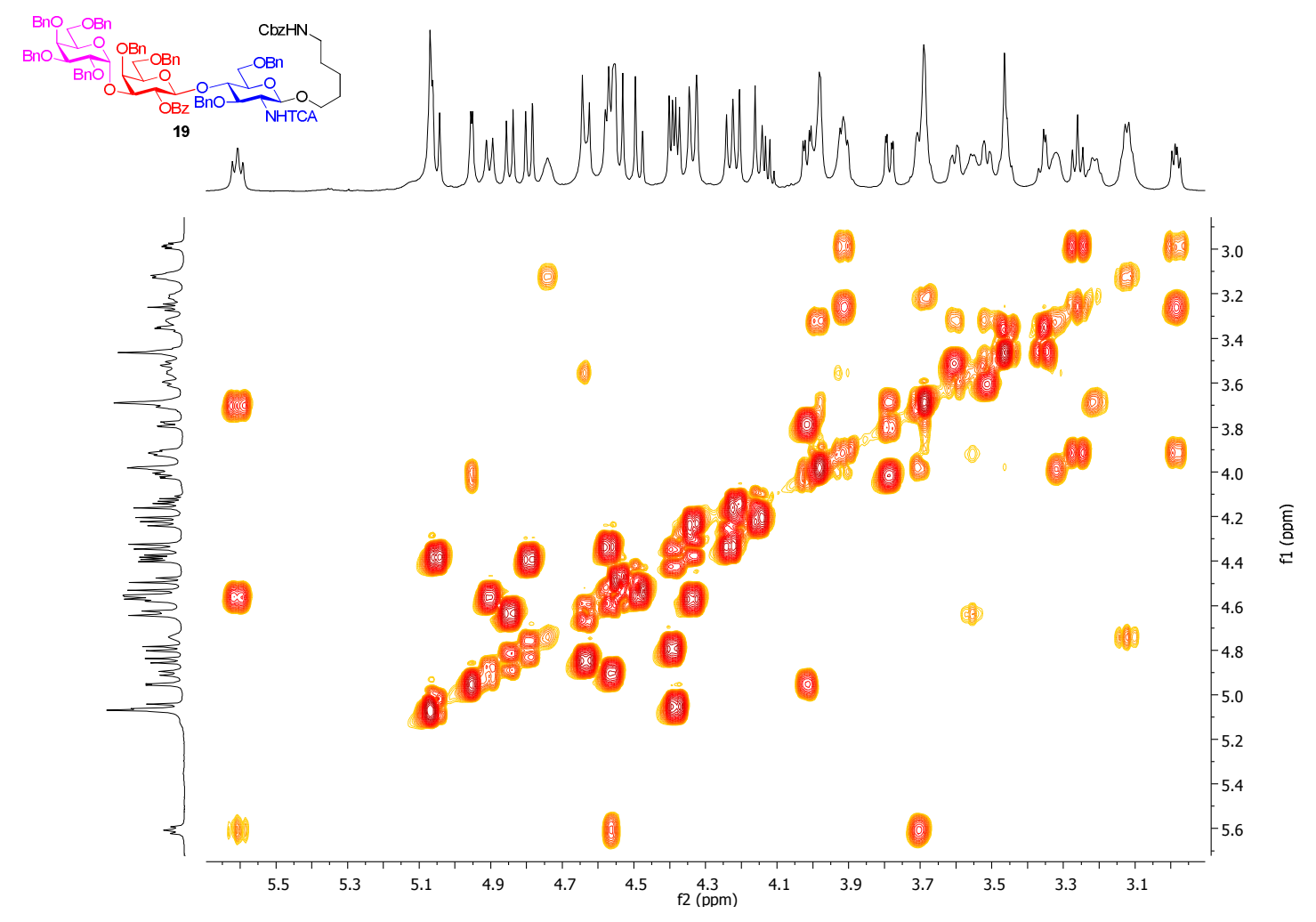


${ }^{1} \mathrm{H}^{13} \mathrm{C}-\mathrm{HSQC} \mathrm{NMR}, 600 \mathrm{MHz}, \mathrm{CDCl}_{3}$

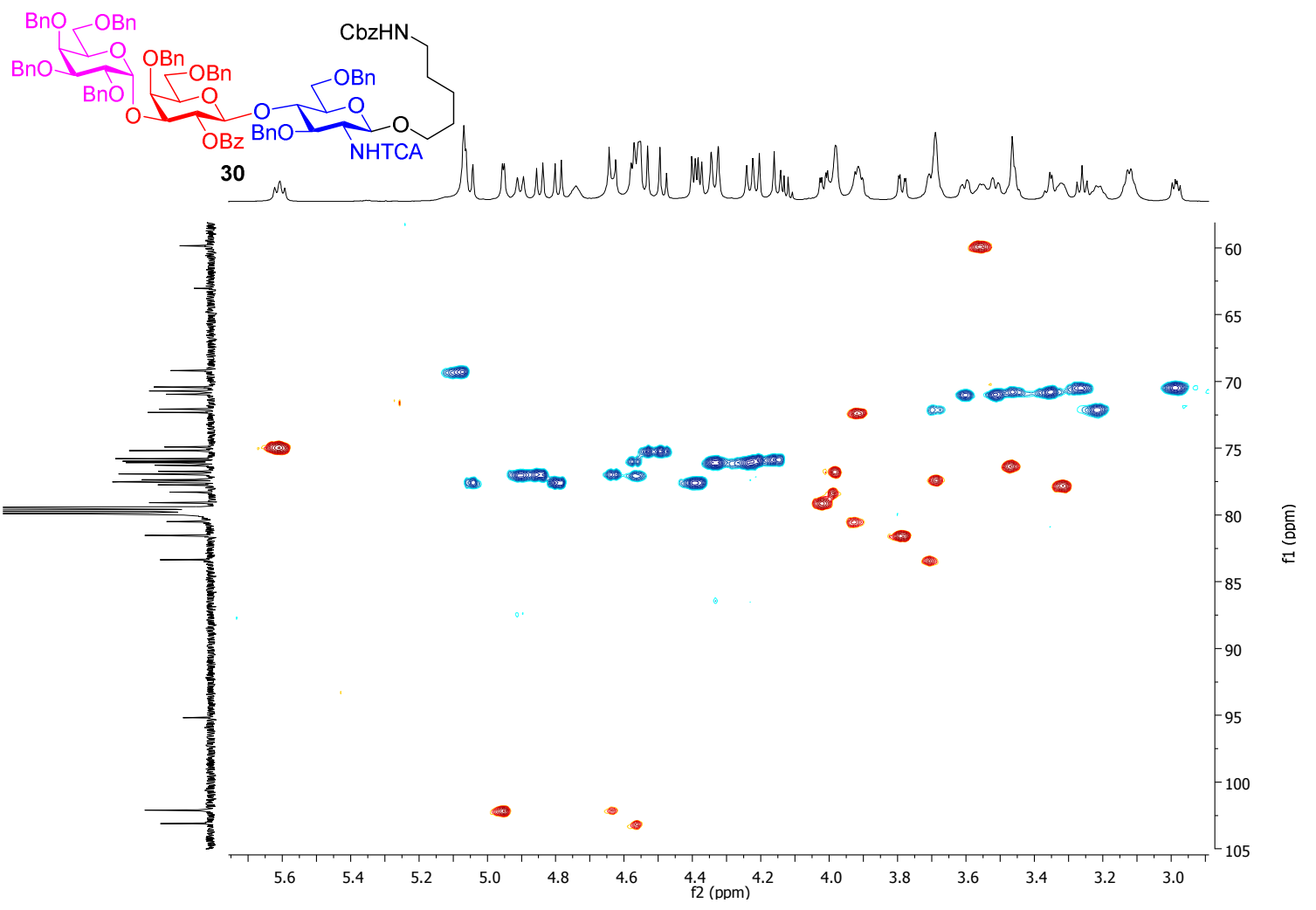

${ }^{1} \mathrm{H}^{13}{ }^{13}$-coupled-HSQC NMR, $600 \mathrm{MHz}, \mathrm{CDCl}_{3}$

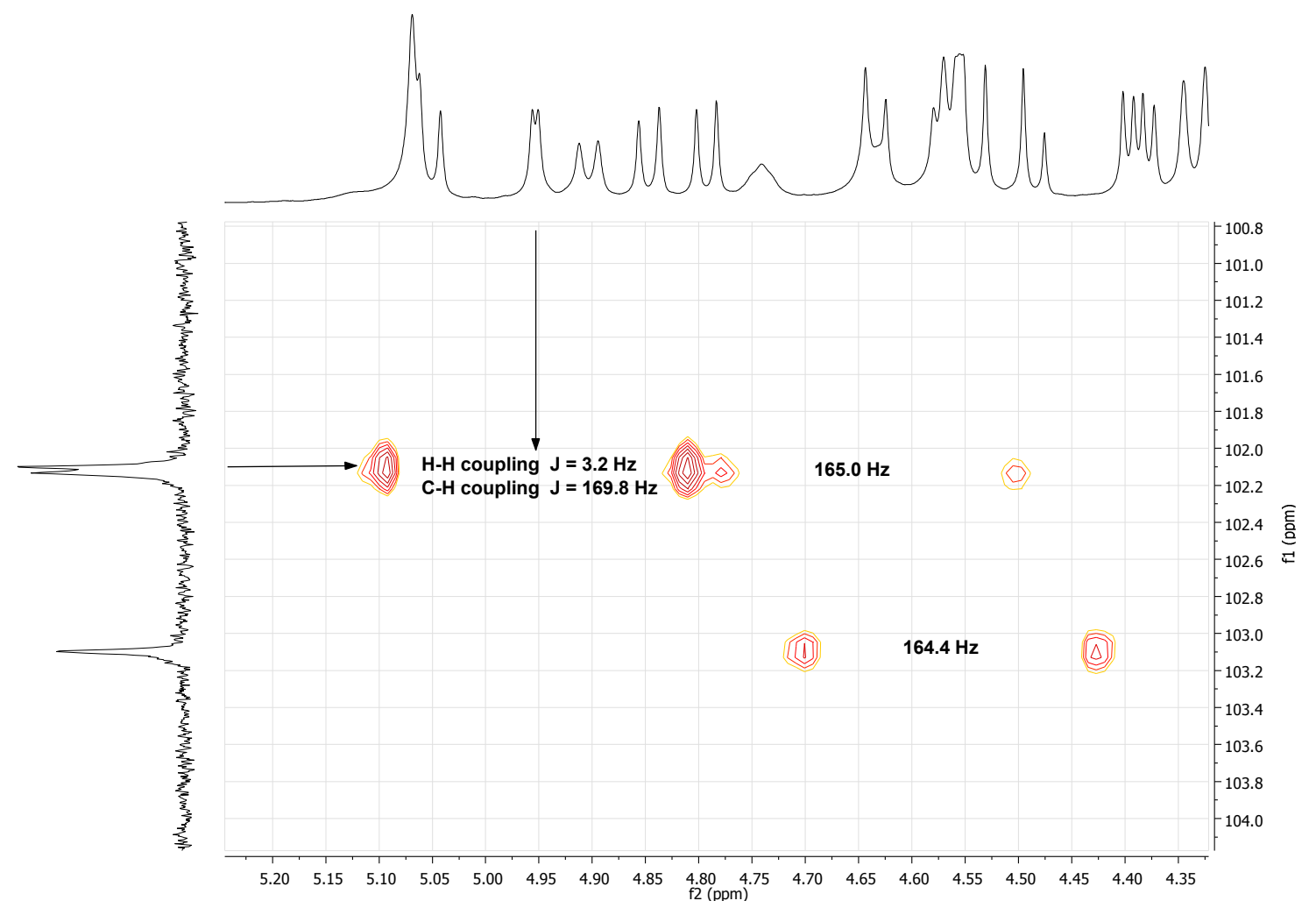




\begin{tabular}{|l|l|l|}
\hline Entry & Sequencce & Ratio $(\alpha / \beta)$ \\
\hline 1 & Gal $1 \rightarrow 3 \mathrm{Gal} \beta 1 \rightarrow 4 \mathrm{Glc} \beta 1 \rightarrow$ linker & 13.7 \\
\hline 2 & Gal $\alpha 1 \rightarrow 3 \mathrm{Gal} \beta 1 \rightarrow 4 \mathrm{GlcNTCA} \beta 1 \rightarrow$ linker & 10.8 \\
\hline 3 & Gal $\alpha 1 \rightarrow 3 \mathrm{Gal} \beta 1 \rightarrow 4 \mathrm{GlcNTCA} \beta 1 \rightarrow 3 \mathrm{Gal} \beta 1 \rightarrow 4 \mathrm{Glc} \beta 1 \rightarrow$ linker & 11.8 \\
\hline
\end{tabular}

Table S2. The acceptor dependency of the formation of 1,2-cis-galactosidic linkages.

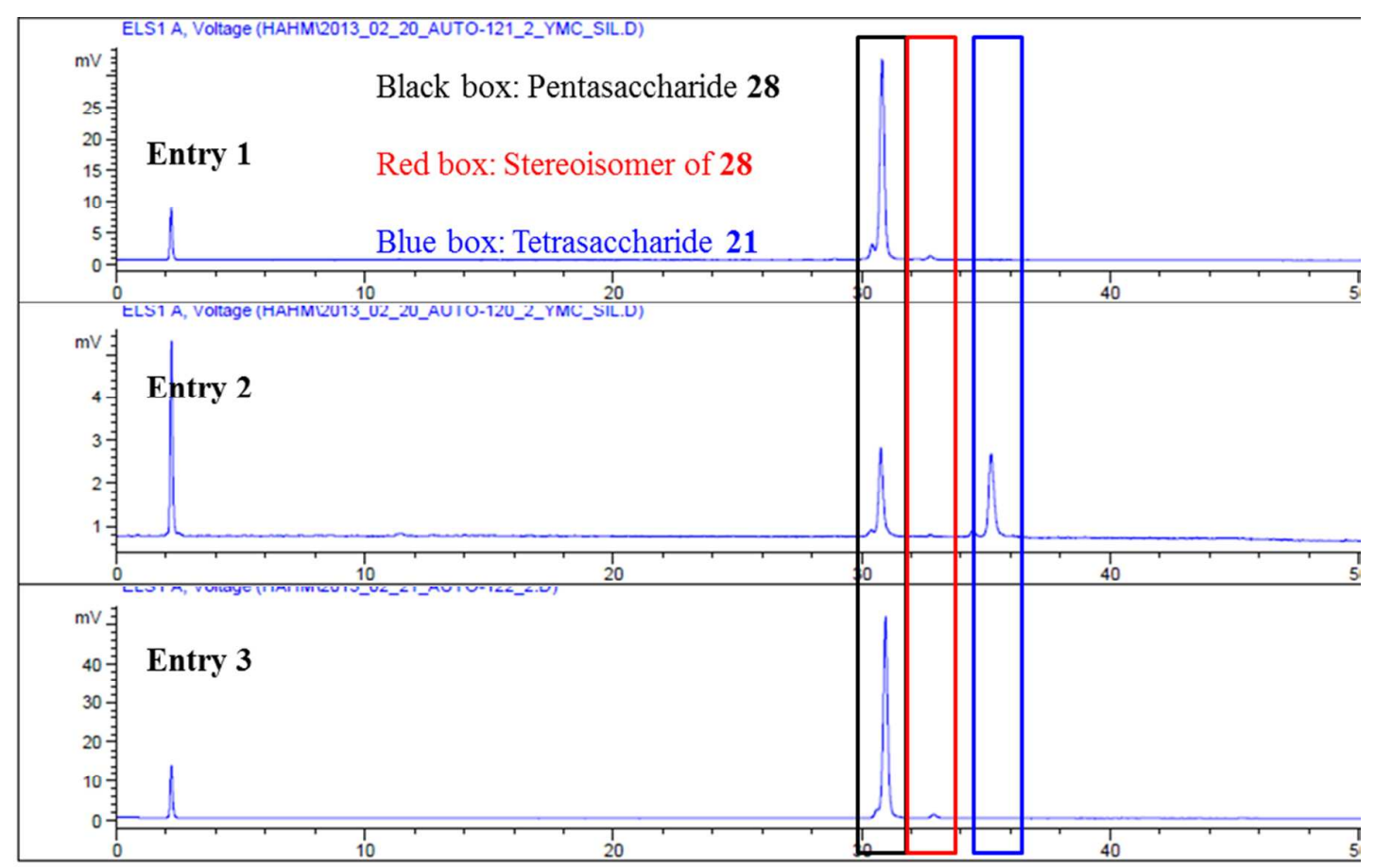

Figure S10. LC-MS of 28. 


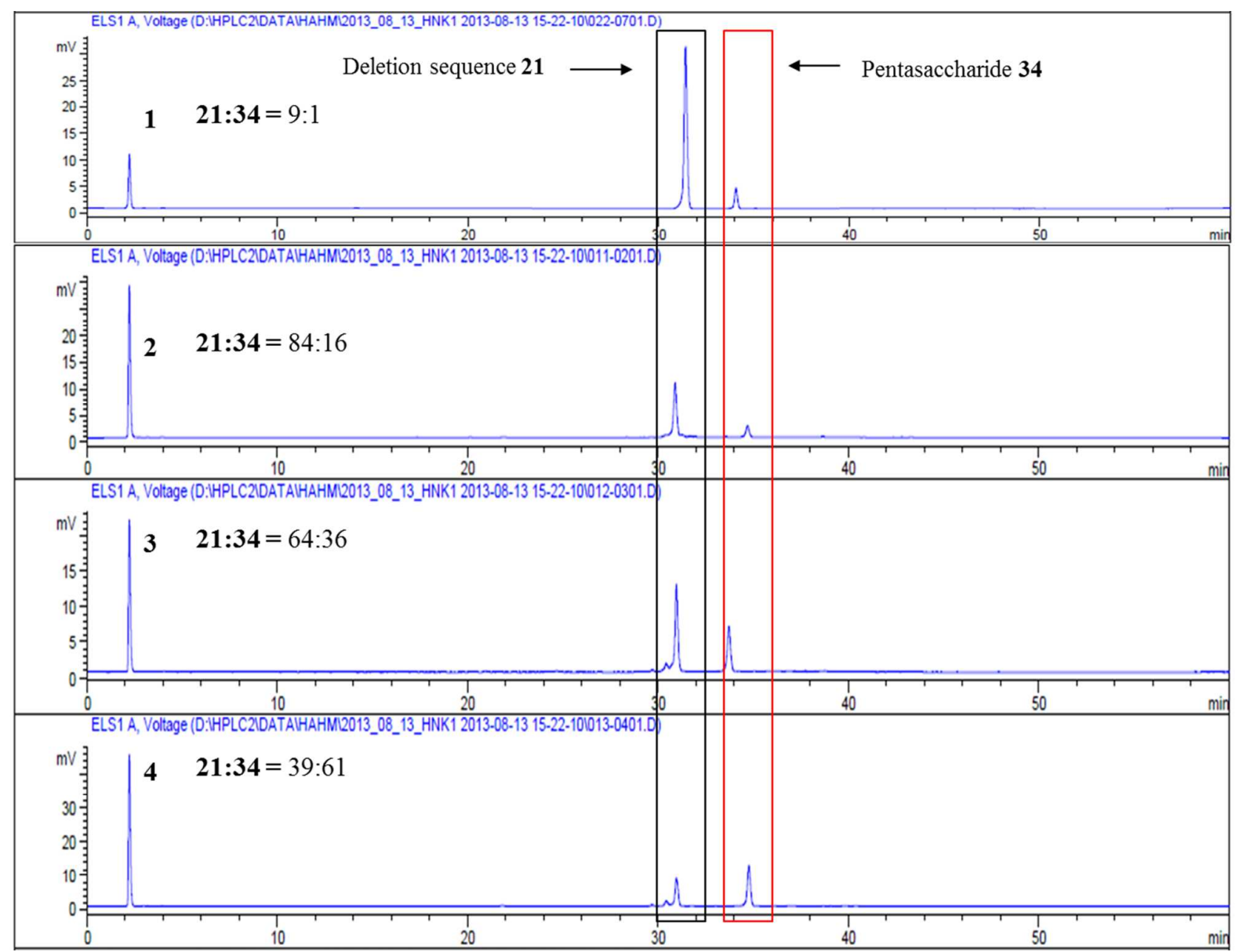

Figure S11. Optimization of automated synthesis of 34 .

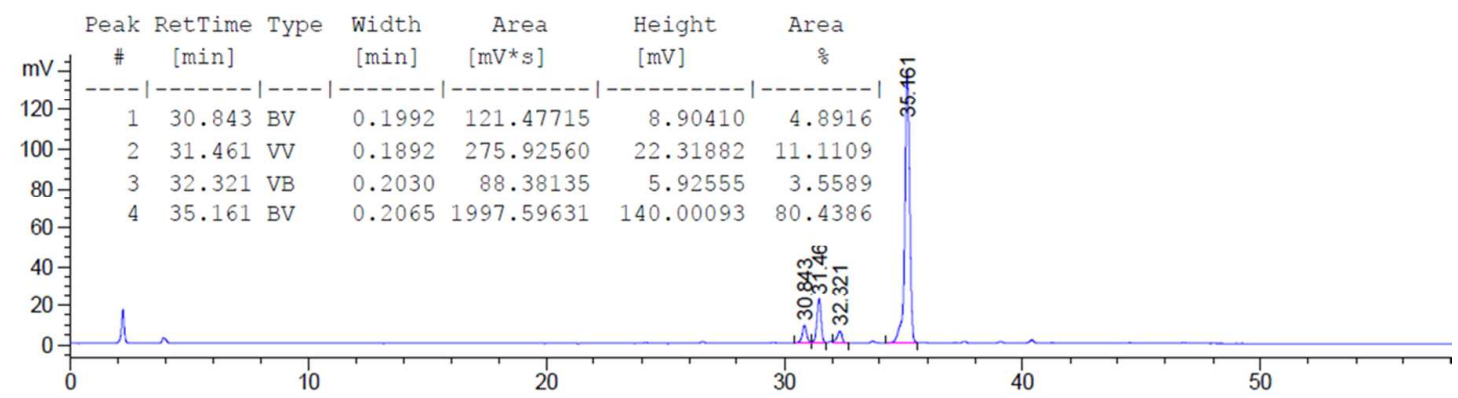

Figure S12. LC-MS of 34. 
${ }^{1} \mathrm{H} \mathrm{NMR}, 600 \mathrm{MHz}, \mathrm{CDCl}_{3}$

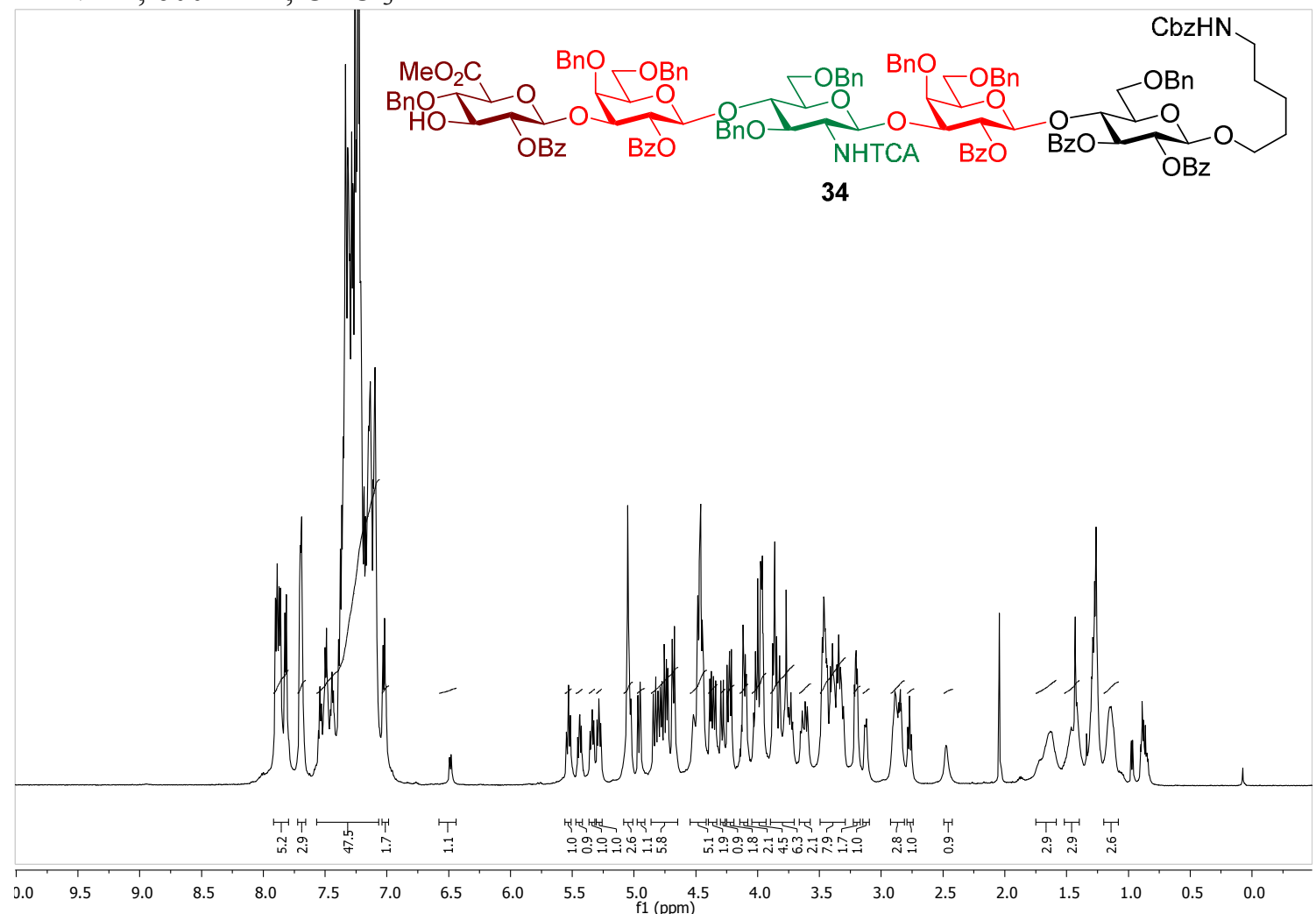

${ }^{13} \mathrm{C} \mathrm{NMR,} 150 \mathrm{MHz}, \mathrm{CDCl}_{3}$

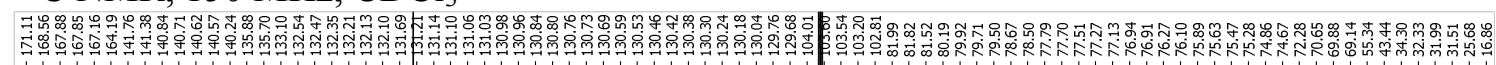

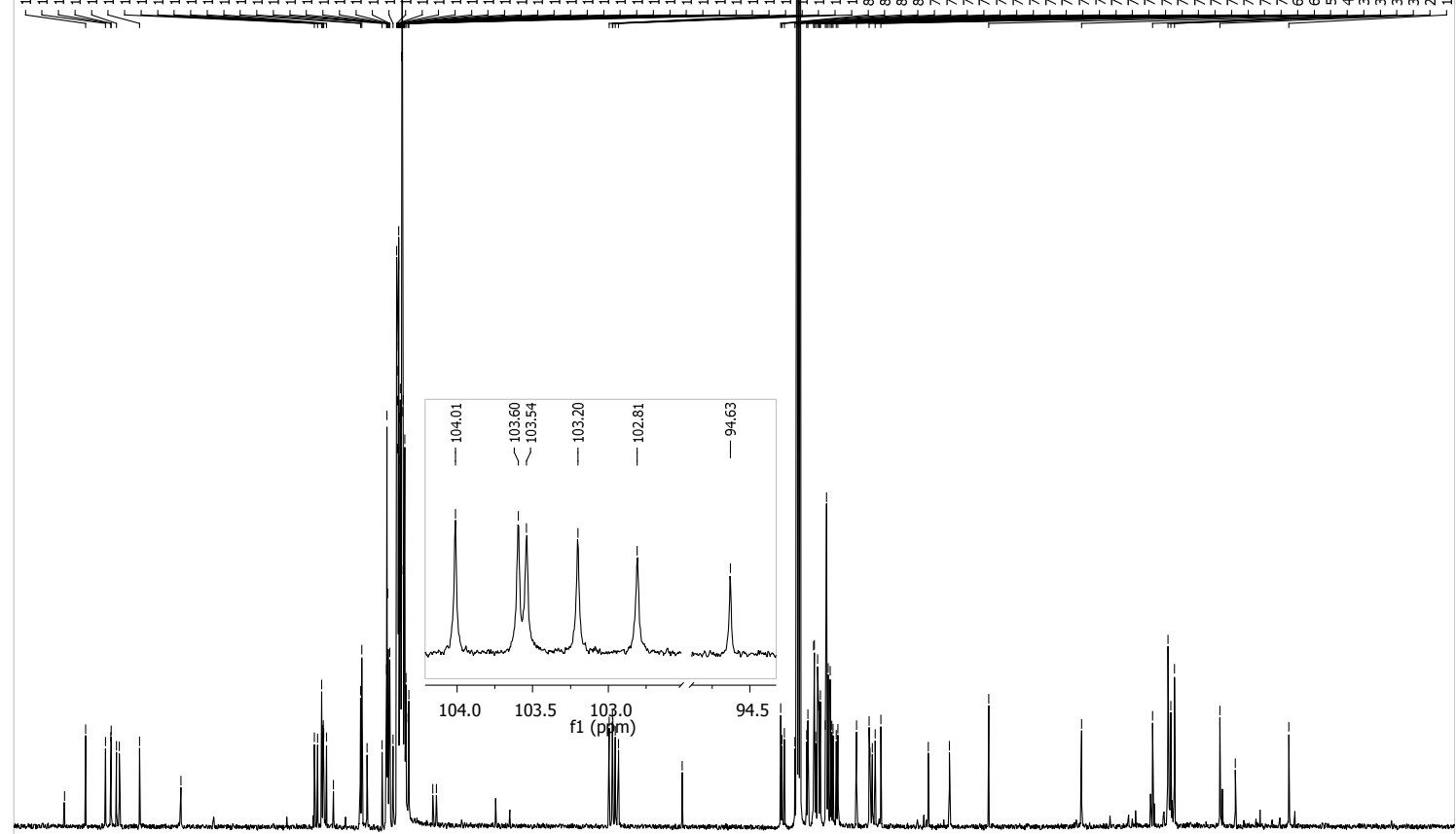

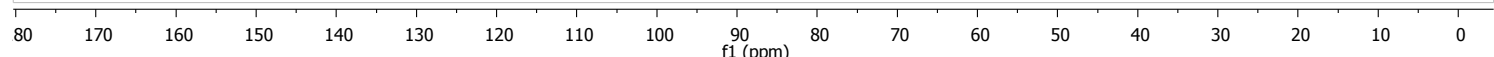


${ }^{1} \mathrm{H}-\mathrm{COSY} \mathrm{NMR}, 600 \mathrm{MHz}, \mathrm{CDCl}_{3}$

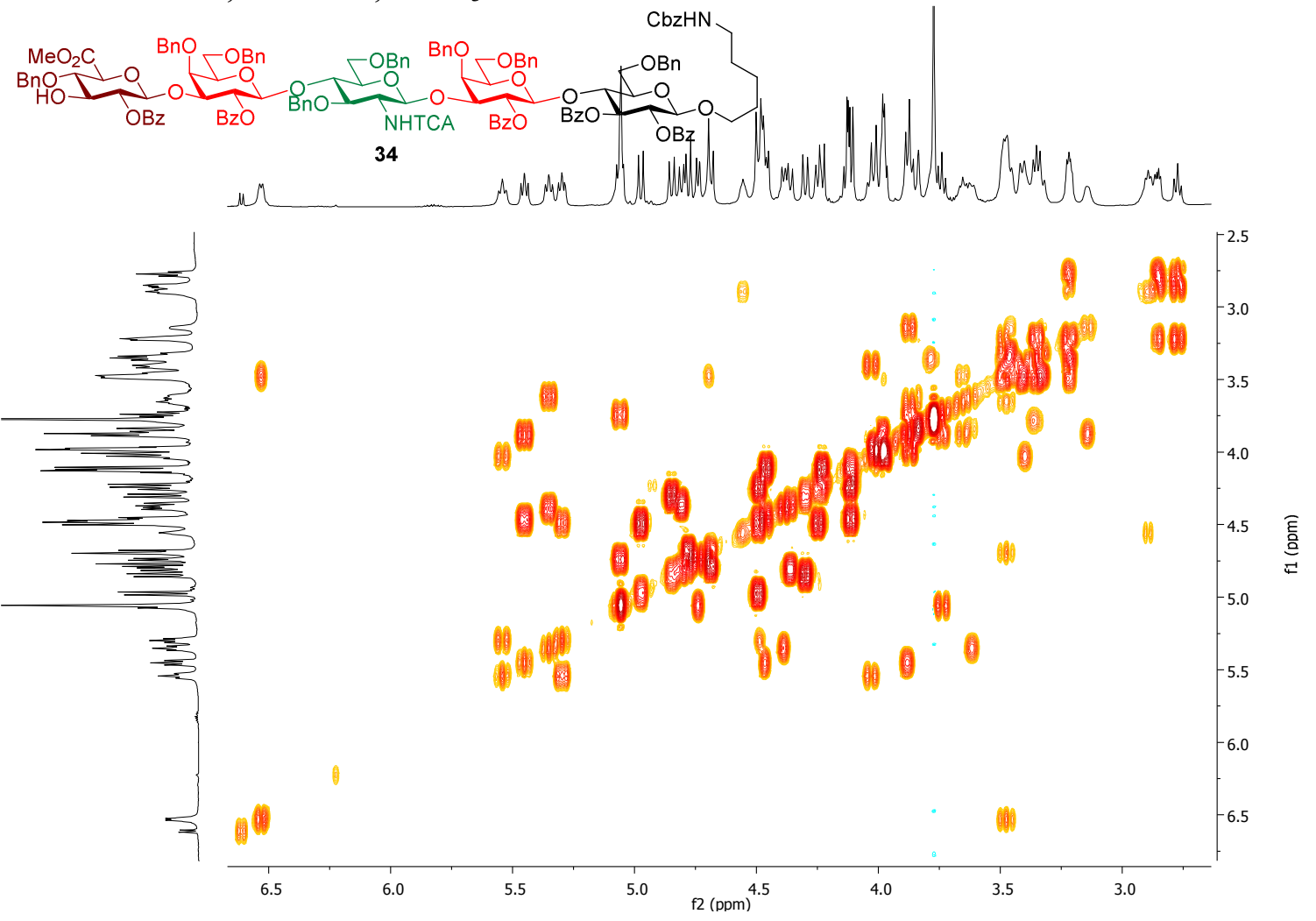

${ }^{1} \mathrm{H}_{-}{ }^{13} \mathrm{C}-\mathrm{HSQC}$ NMR, $600 \mathrm{MHz}, \mathrm{CDCl}_{3}$

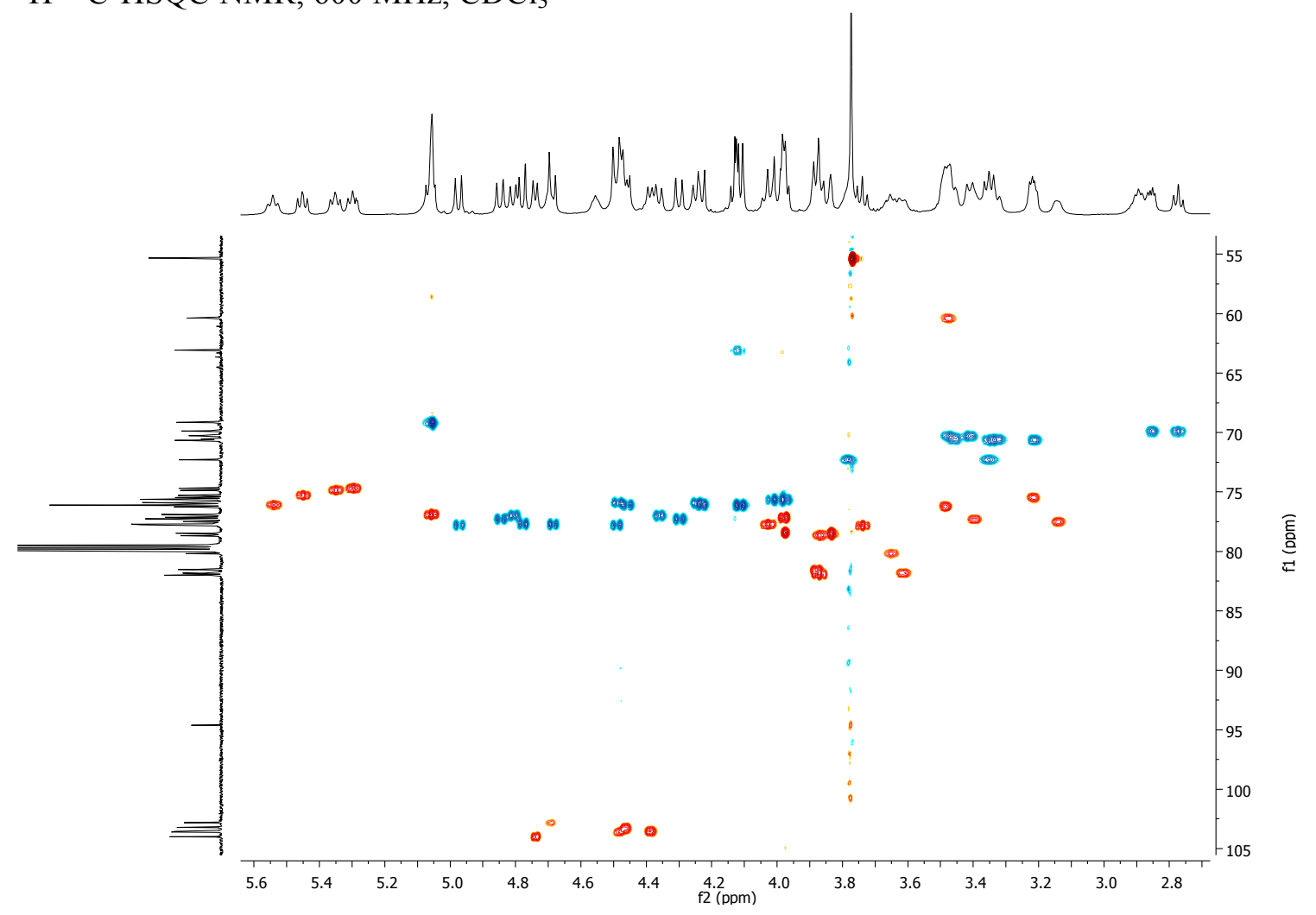




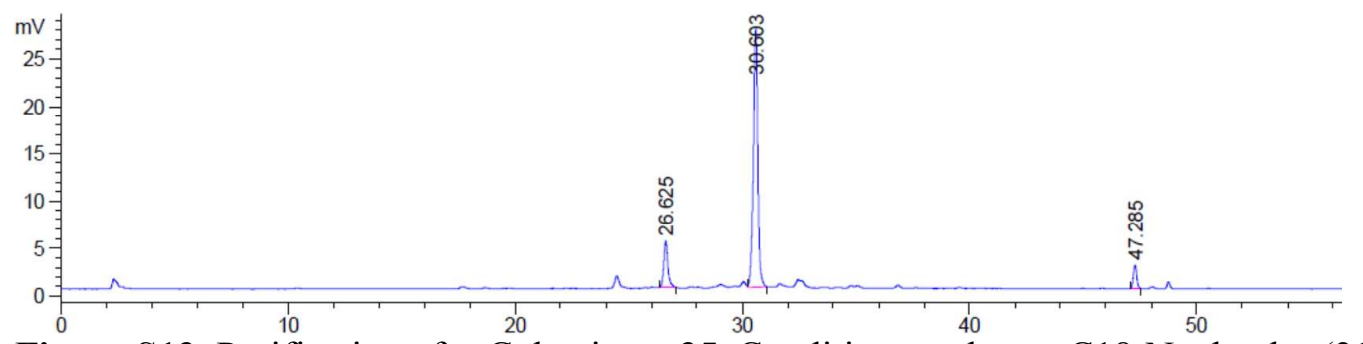

Figure S13. Purification of $\alpha$-Gal epitope 35. Conditions: column: C18-Nucleodur $(21 \times 250 \mathrm{~mm} ; 5$ $\mu \mathrm{m}$ ); flow rate: $10 \mathrm{~mL} \cdot \mathrm{min}^{-1}$; eluents: $0.01 \mathrm{M} \mathrm{NH}_{4} \mathrm{HCO}_{3}$ in water/MeCN; gradient: $45 \%$ (5 $\min$ ) $\rightarrow 55 \%$ (in $40 \mathrm{~min}$ ) $\rightarrow 100 \%$ (in $5 \mathrm{~min}$ ); detection: ELSD.

${ }^{1} \mathrm{H} \mathrm{NMR}, 600 \mathrm{MHz}, \mathrm{CDCl}_{3}$

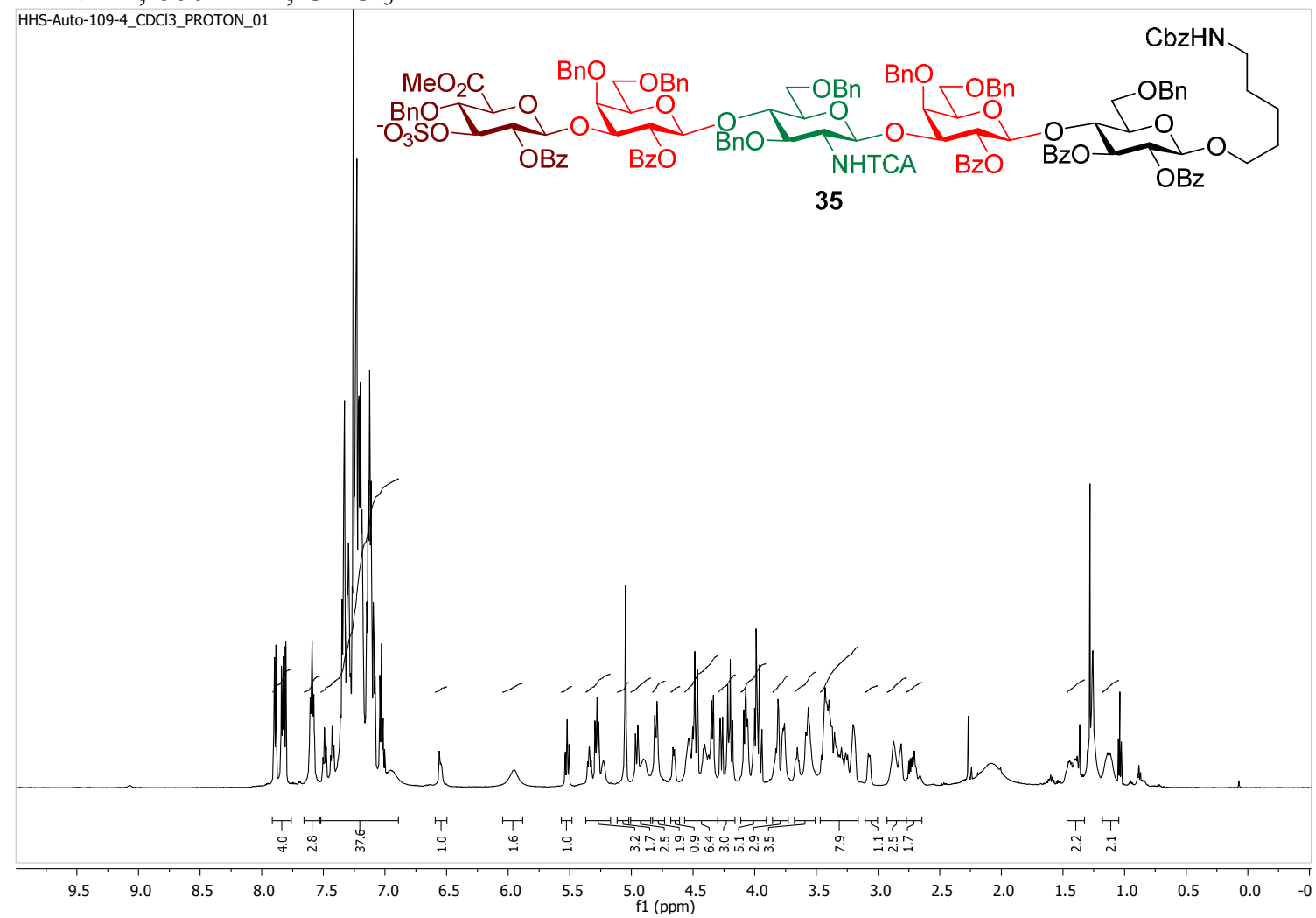


${ }^{13} \mathrm{C}$ NMR, $150 \mathrm{MHz}, \mathrm{MeOH}-\mathrm{d}_{4}$

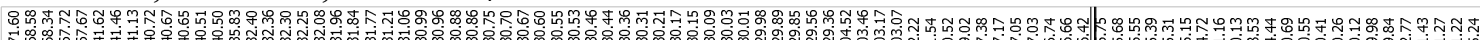

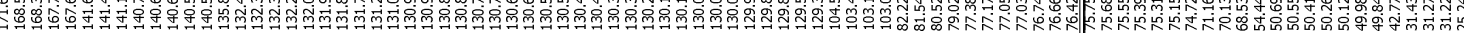
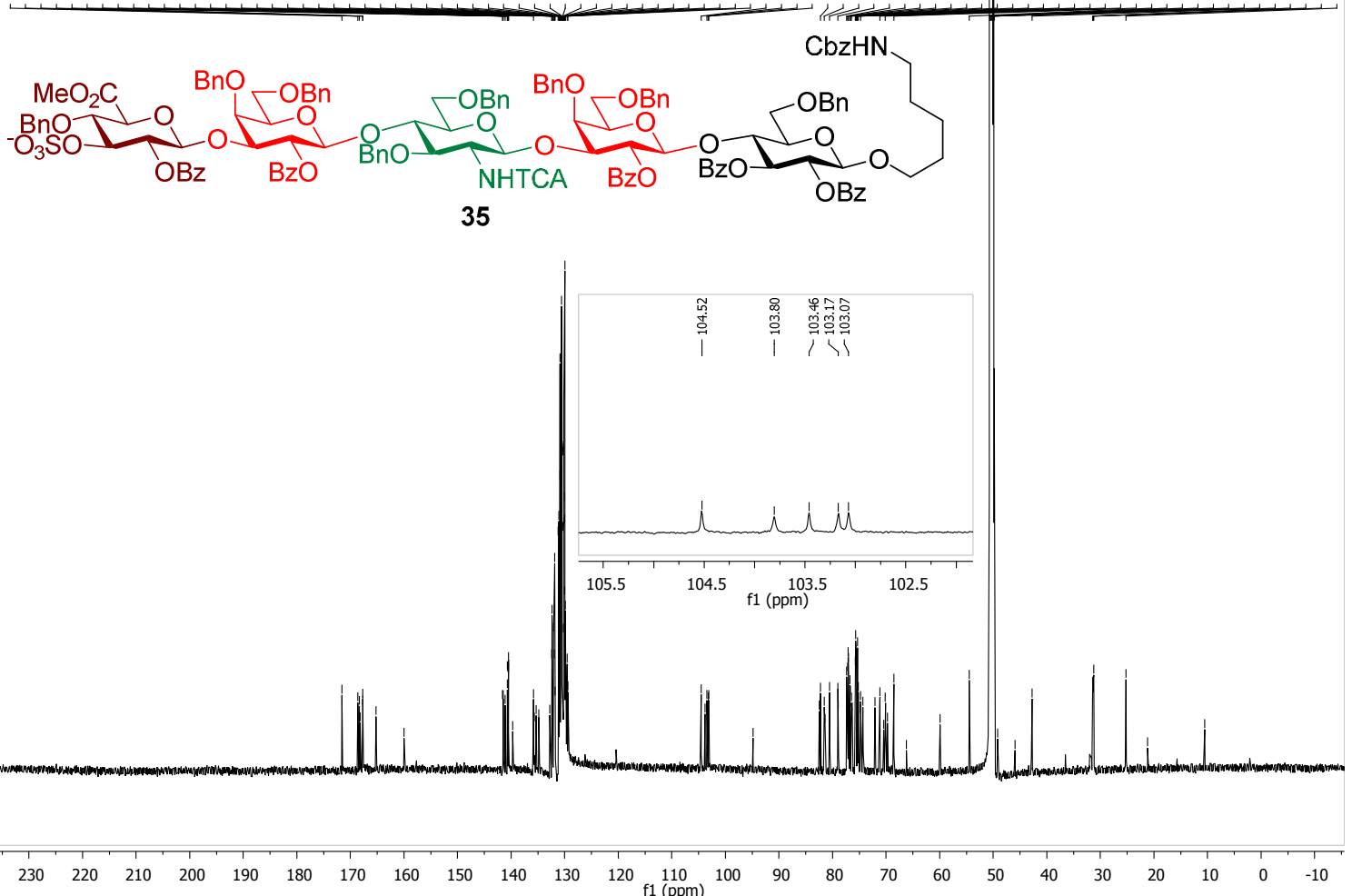

${ }^{1} \mathrm{H}-\mathrm{COSY} \mathrm{NMR}, 600 \mathrm{MHz}, \mathrm{MeOH}-\mathrm{d}_{4}$

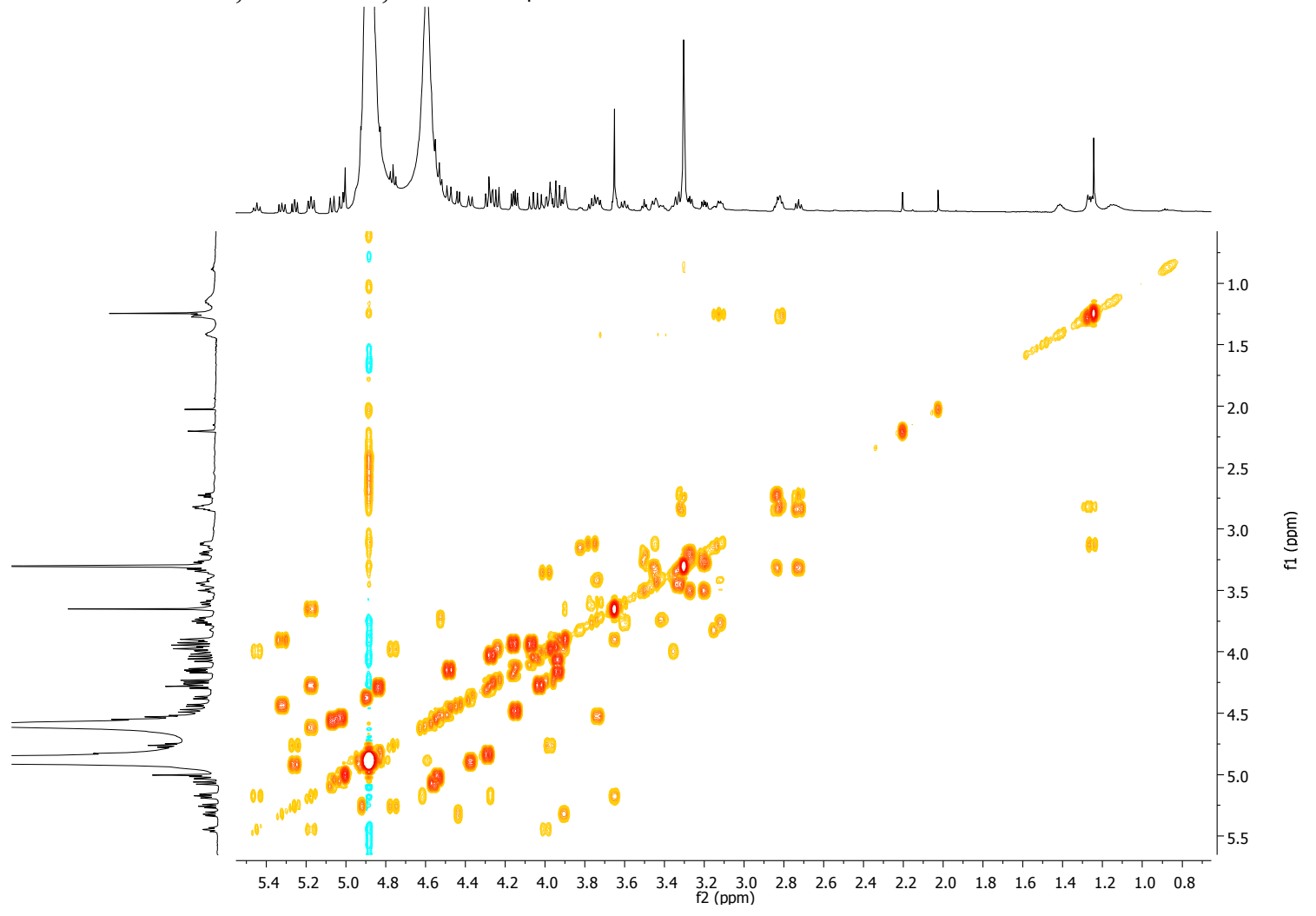


${ }^{1} \mathrm{H}_{-}{ }^{13} \mathrm{C}-\mathrm{HSQC}$ NMR, $600 \mathrm{MHz}, \mathrm{MeOH}-\mathrm{d}_{4}$

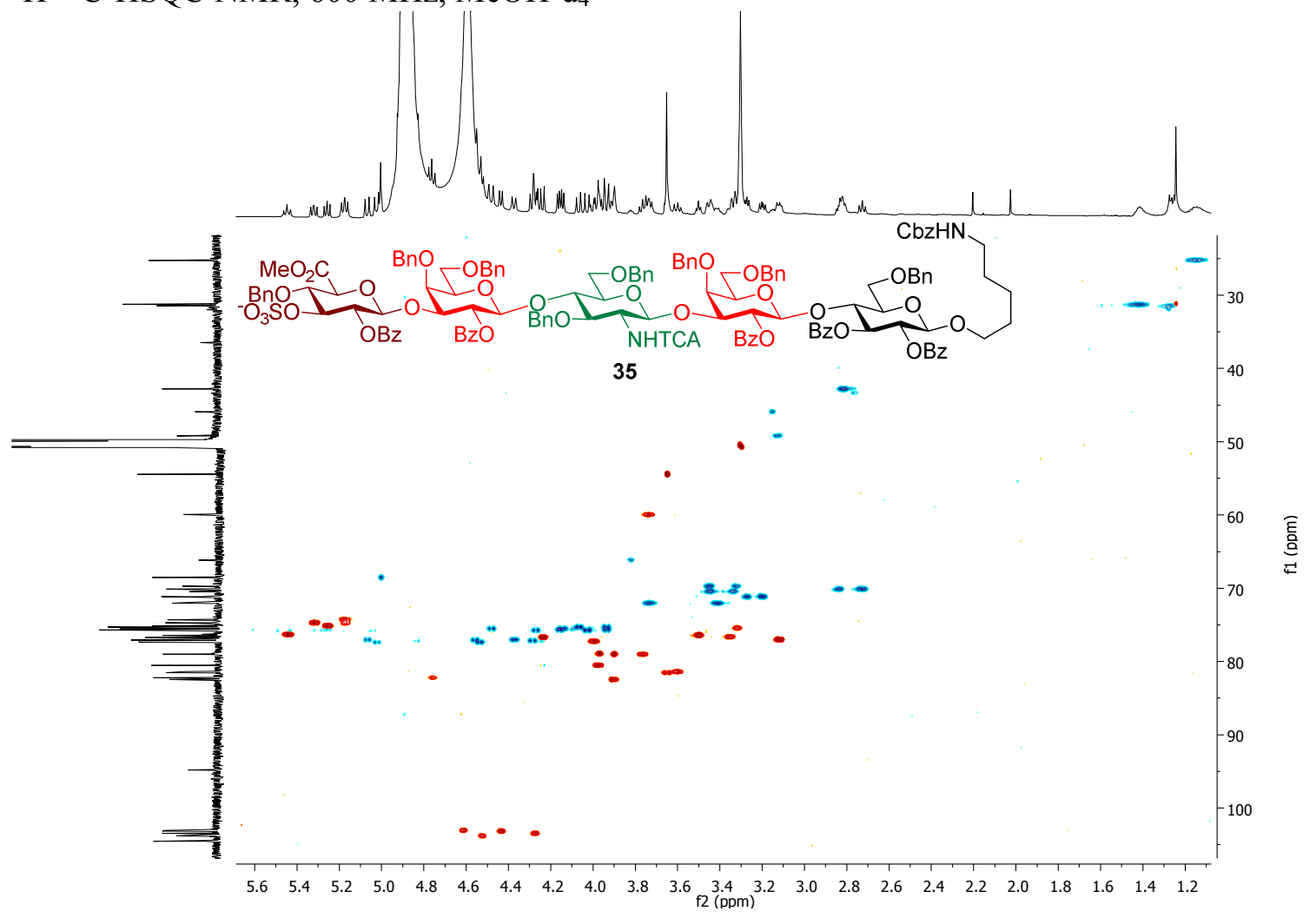

non-sulfated pentasaccharide 34

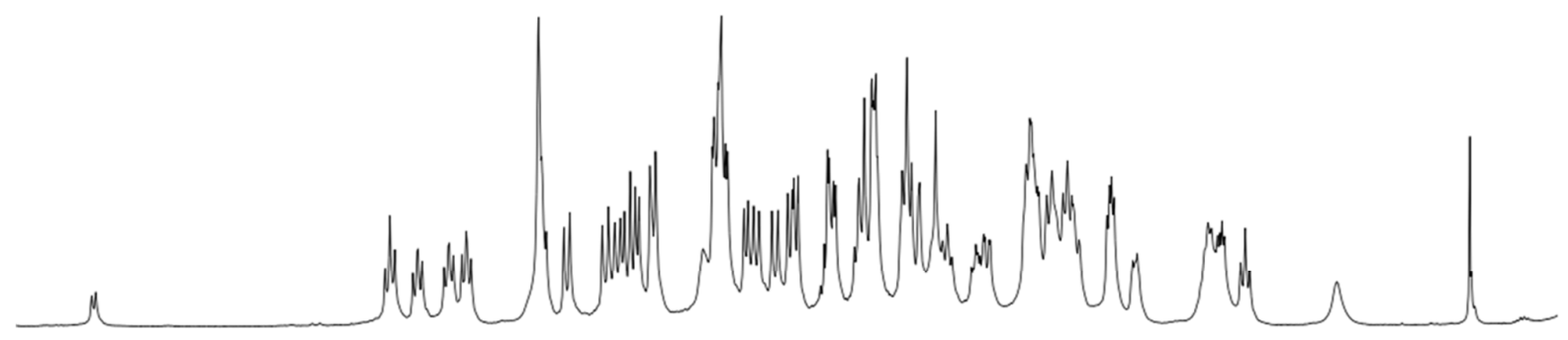

sulfated pentasaccharide 35
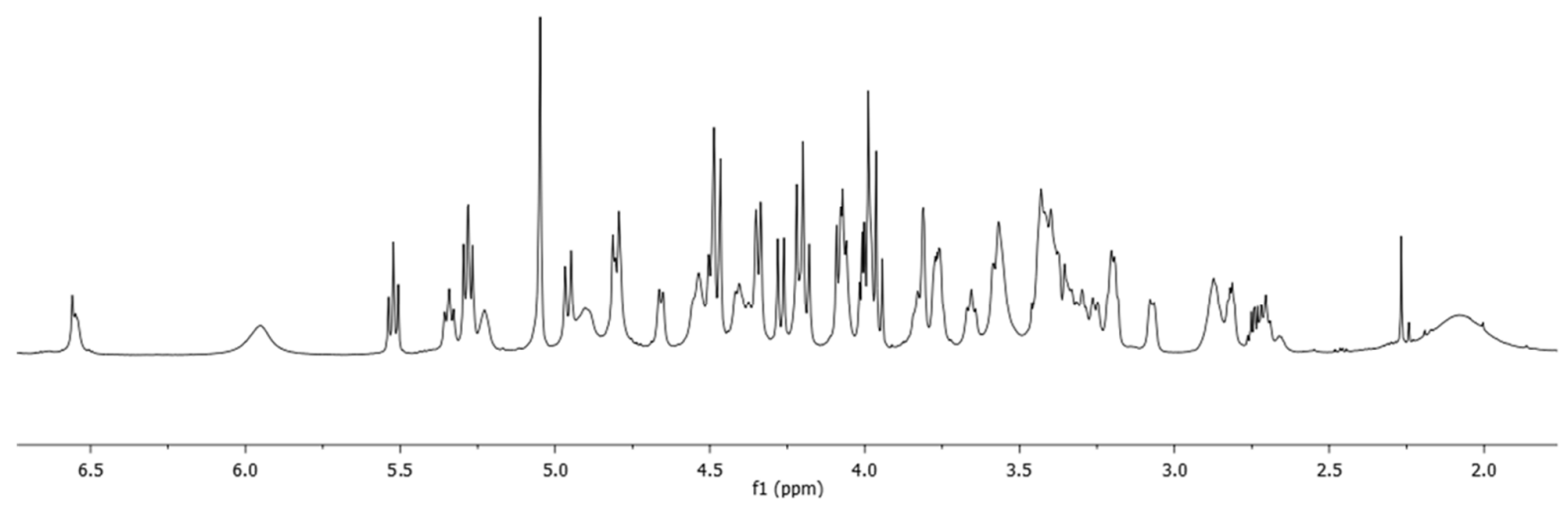
${ }^{1} \mathrm{H} \mathrm{NMR}, 600 \mathrm{MHz}, \mathrm{D}_{2} \mathrm{O}$

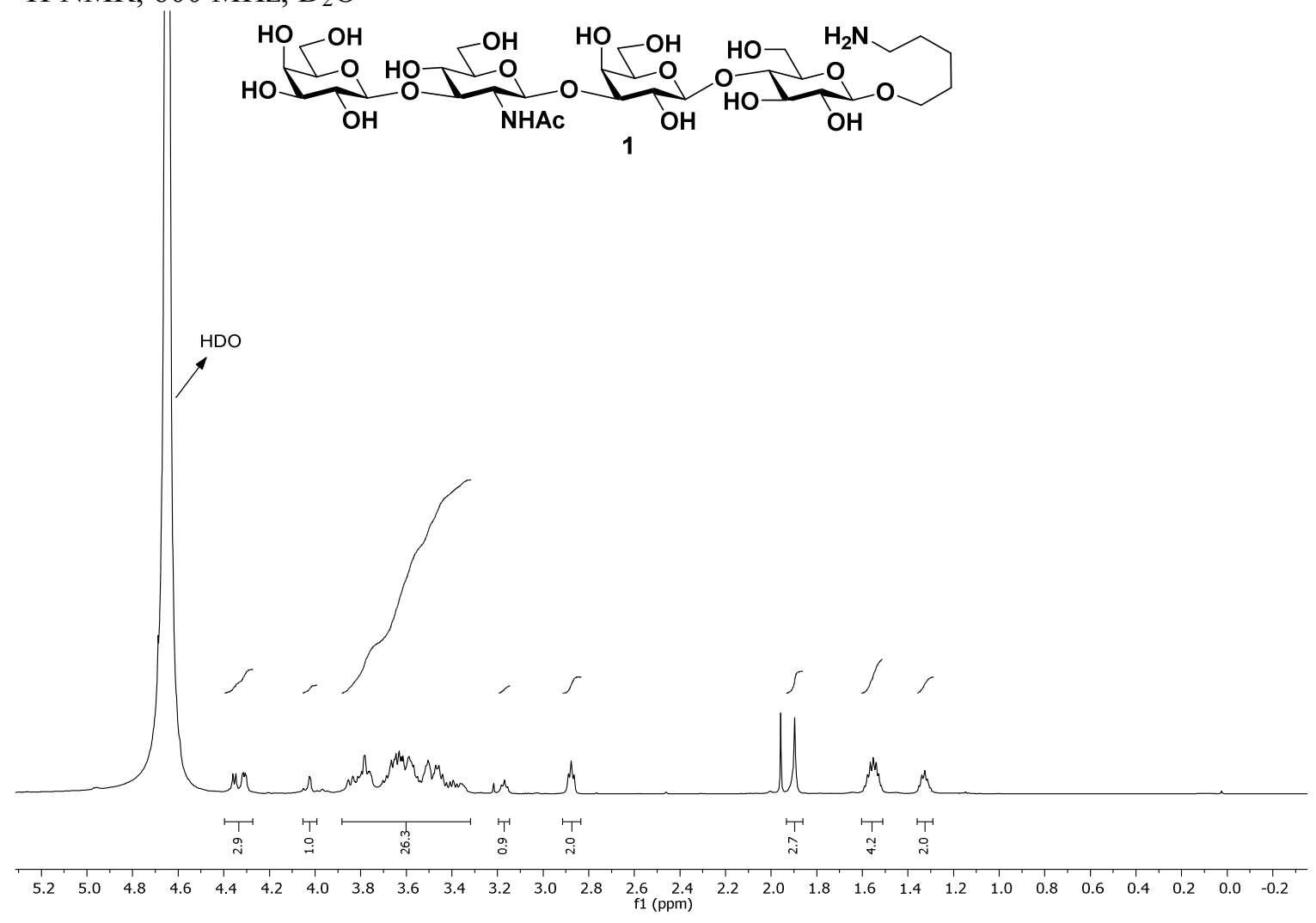

${ }^{13} \mathrm{C}$ NMR, $150 \mathrm{MHz}, \mathrm{D}_{2} \mathrm{O}$

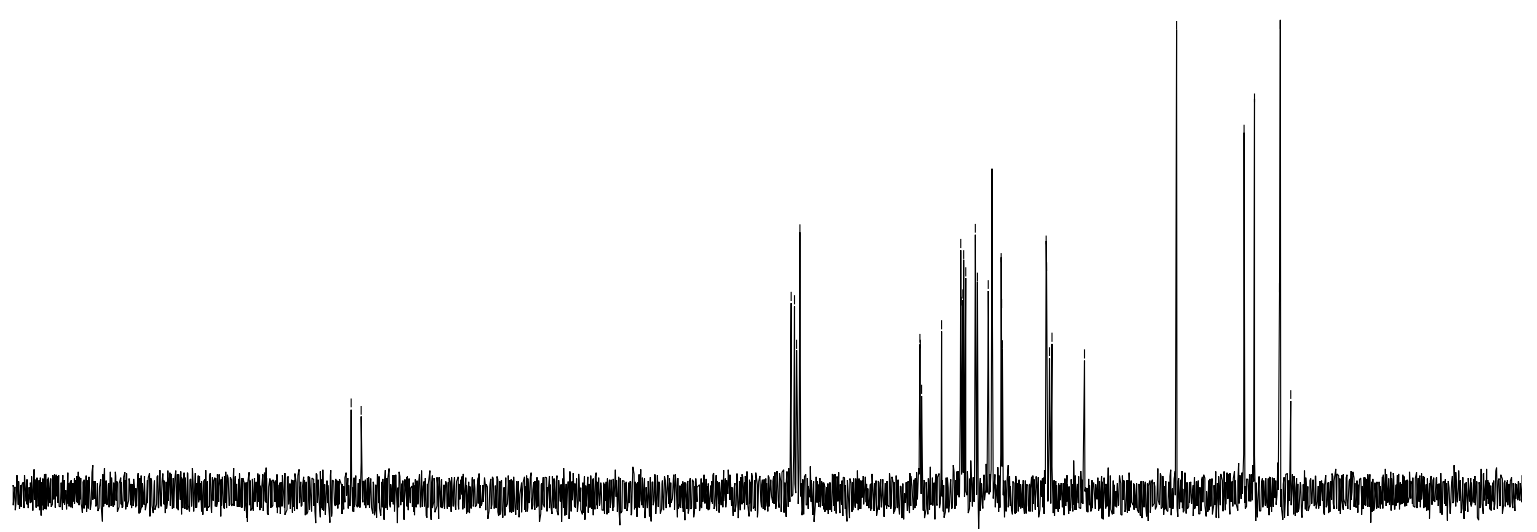

$\begin{array}{lllllllllllllllllllllllll}230 & 220 & 210 & 200 & 190 & 180 & 170 & 160 & 150 & 140 & 130 & 120 & 110 & 100 & 90 & 80 & 70 & 60 & 50 & 40 & 30 & 20 & 10 & 0 & -10\end{array}$ 
${ }^{1} \mathrm{H}$ NMR, $600 \mathrm{MHz}, \mathrm{D}_{2} \mathrm{O}$

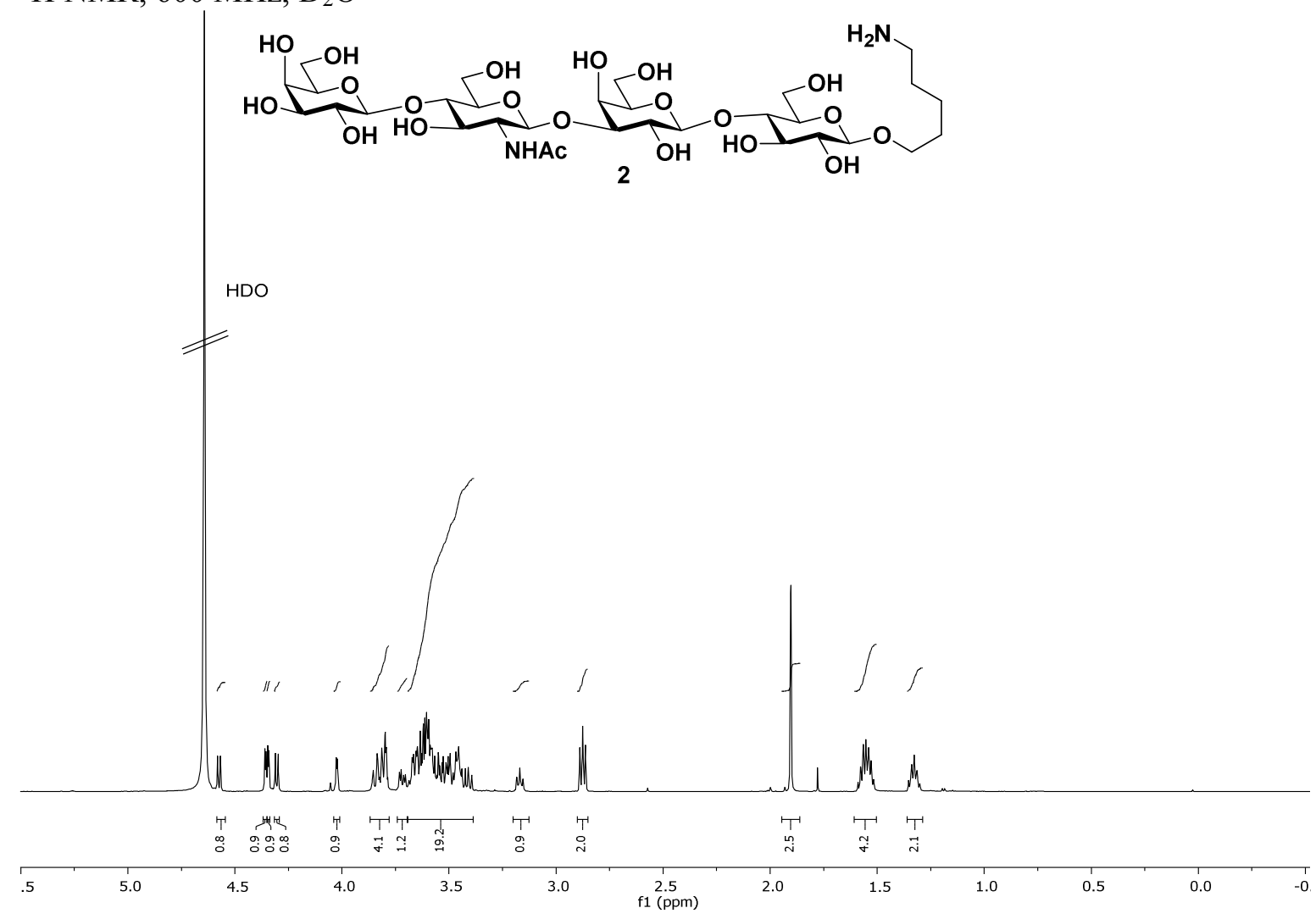

${ }^{13} \mathrm{C}$ NMR, $150 \mathrm{MHz}, \mathrm{D}_{2} \mathrm{O}$
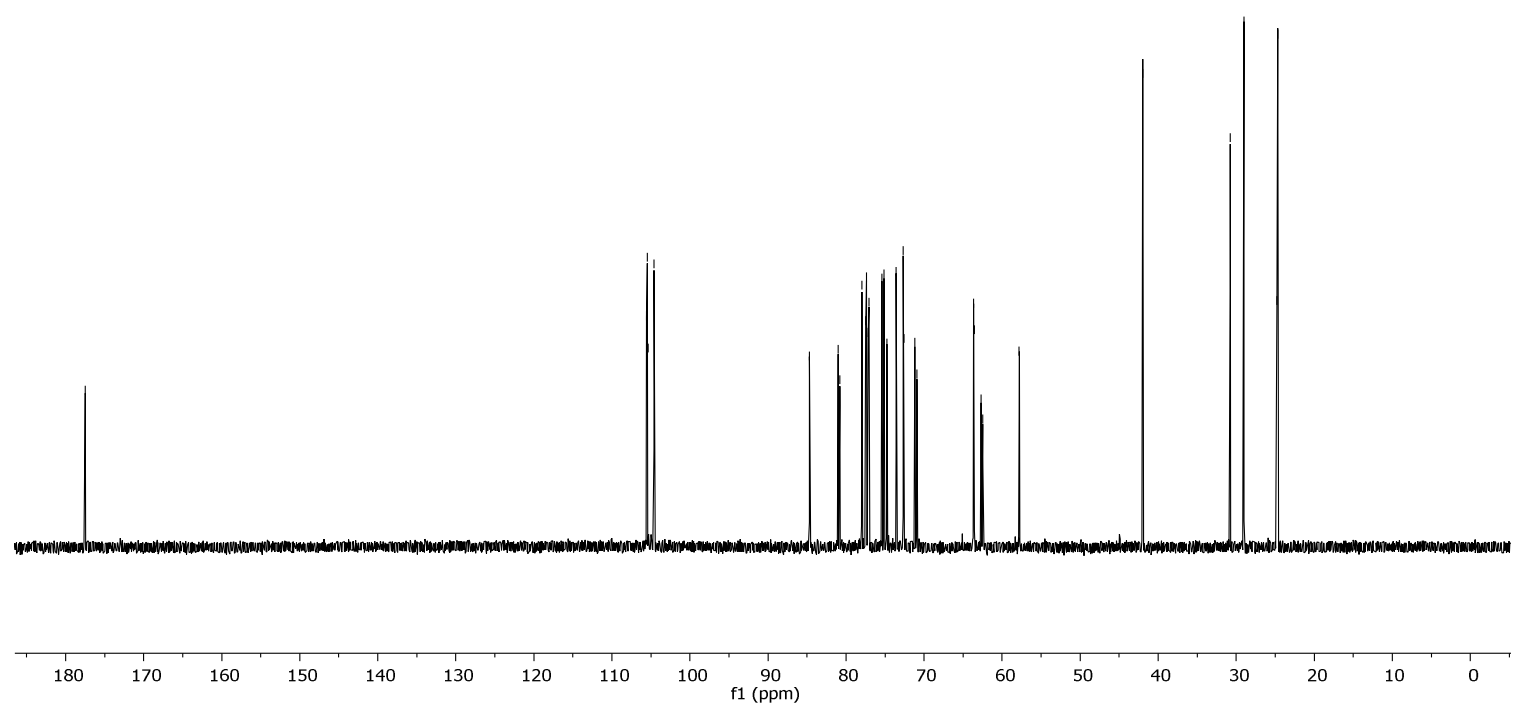
${ }^{1} \mathrm{H} \mathrm{NMR}, 600 \mathrm{MHz}, \mathrm{D}_{2} \mathrm{O}$

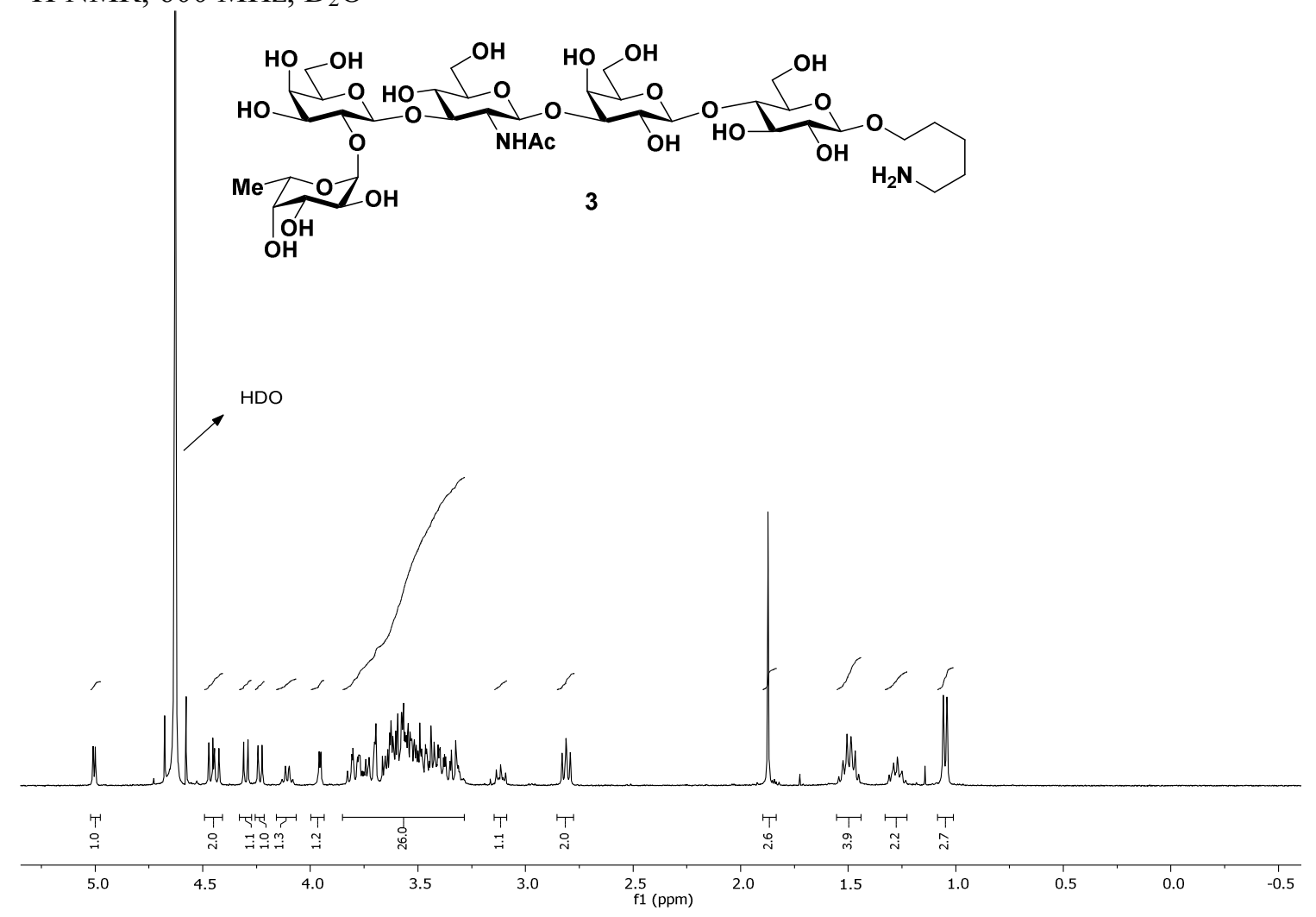

${ }^{13} \mathrm{C}$ NMR, $150 \mathrm{MHz}, \mathrm{D}_{2} \mathrm{O}$

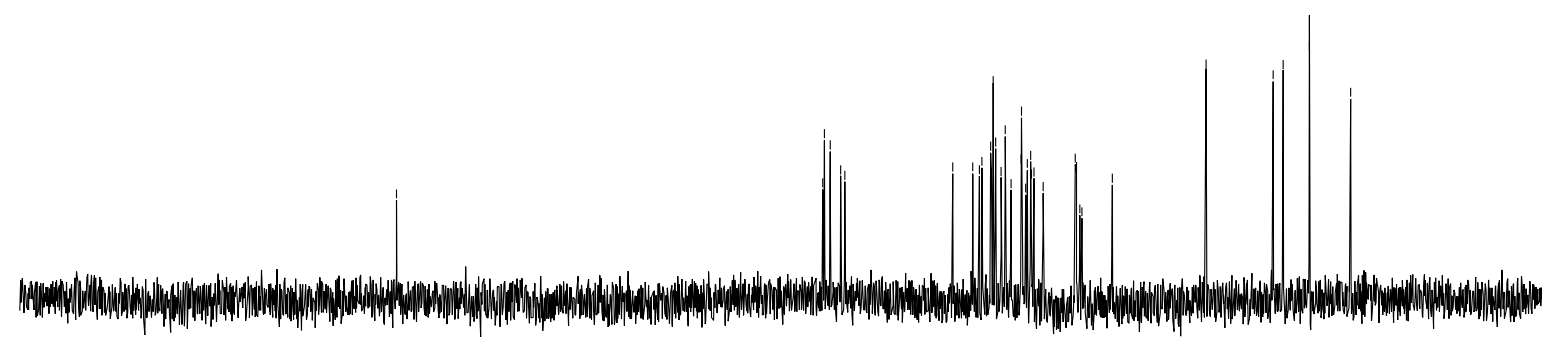

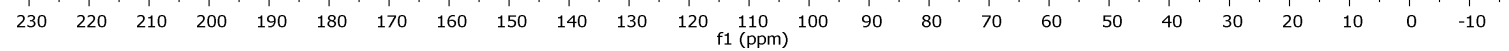


${ }^{1} \mathrm{H}$ NMR, $600 \mathrm{MHz}, \mathrm{D}_{2} \mathrm{O}$

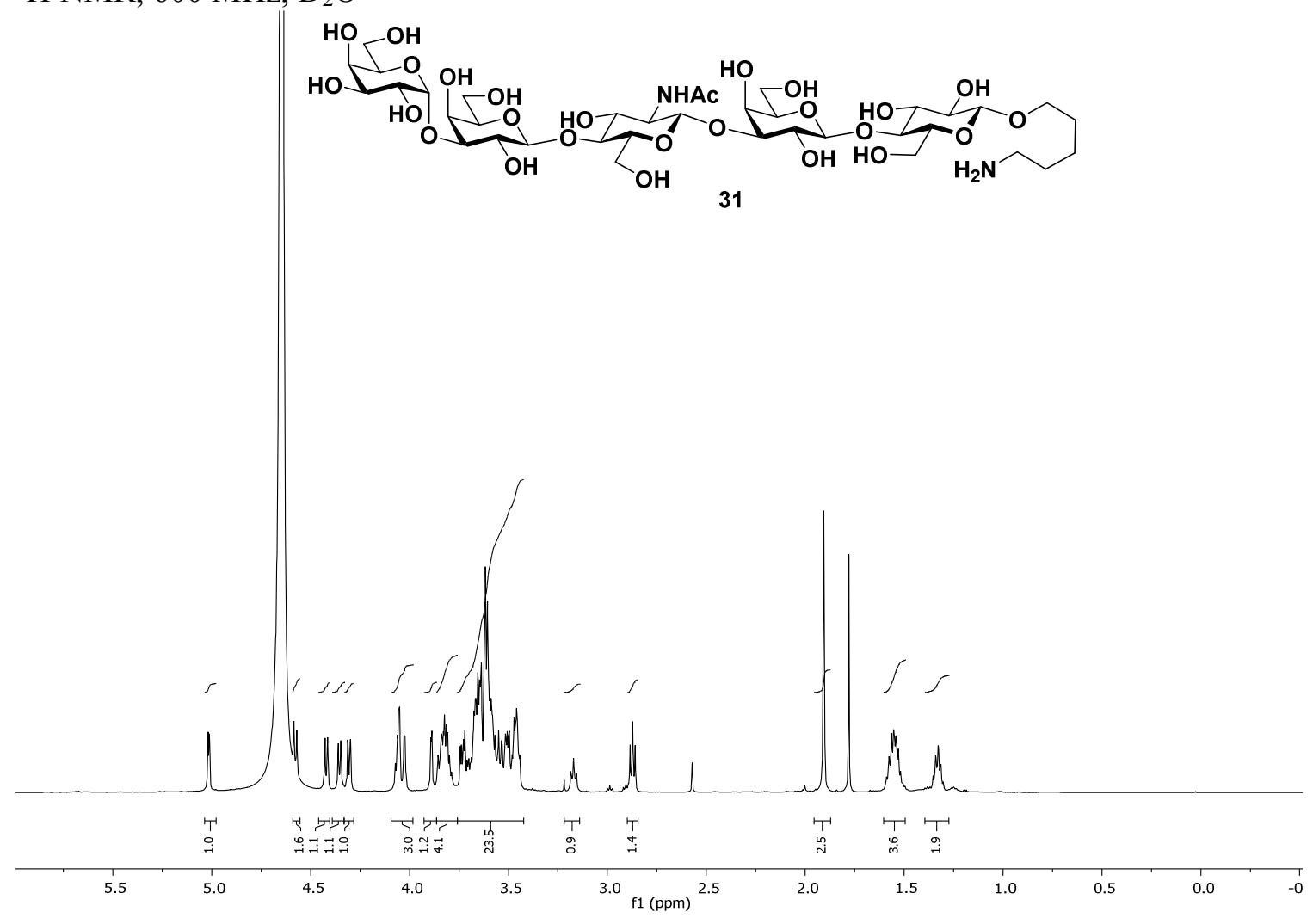

${ }^{13} \mathrm{C}$ NMR, $150 \mathrm{MHz}, \mathrm{D}_{2} \mathrm{O}$ 
${ }^{1} \mathrm{H}$ NMR, $600 \mathrm{MHz}, \mathrm{D}_{2} \mathrm{O}$
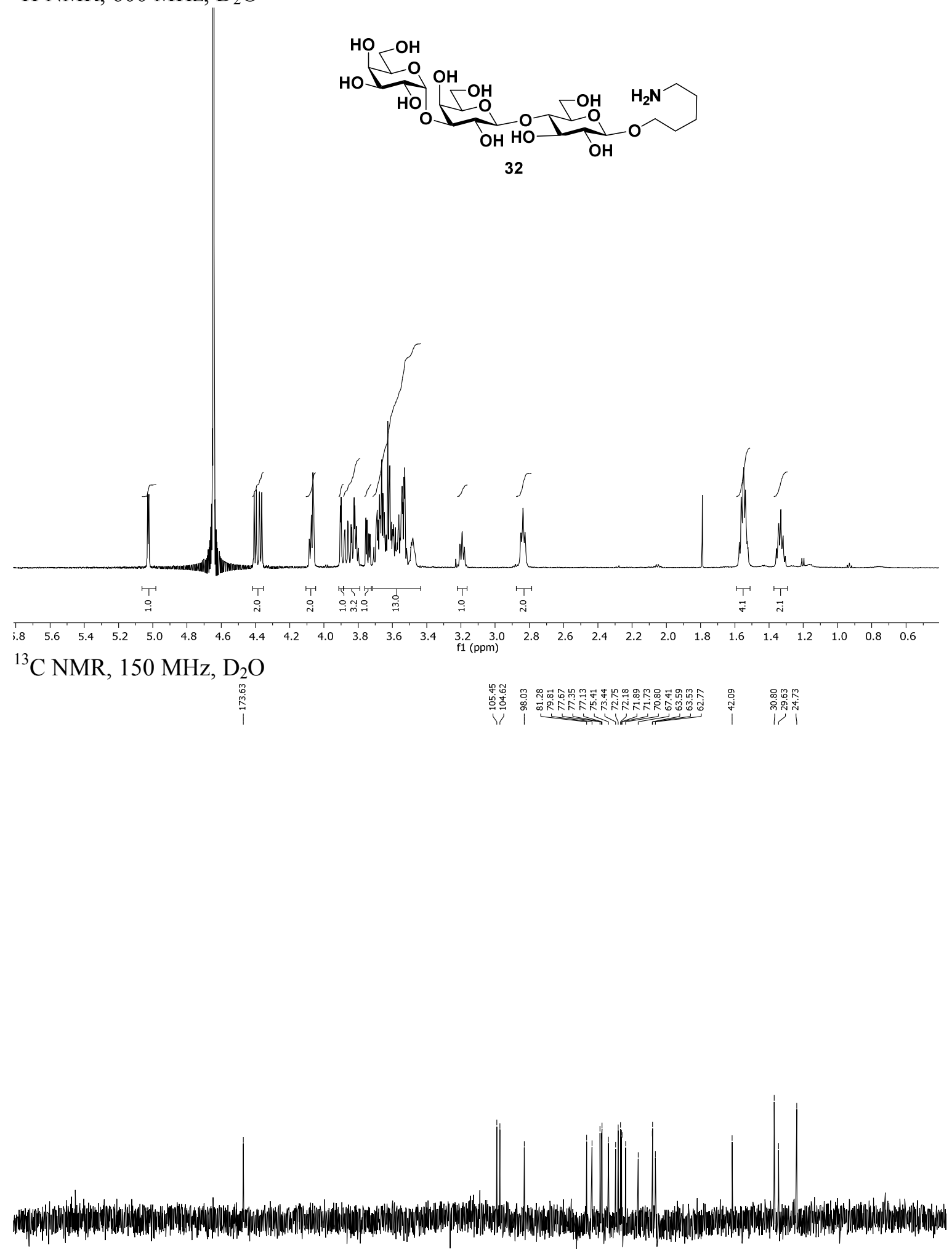

$\begin{array}{llllllllllllllllllllllllllllllll}230 & 220 & 210 & 200 & 190 & 180 & 170 & 160 & 150 & 140 & 130 & 120 & 110 & 100 & 90 & 80 & 70 & 60 & 50 & 40 & 30 & 20 & 10 & 0 & -10\end{array}$ 
${ }^{1} \mathrm{H}$ NMR, $600 \mathrm{MHz}, \mathrm{D}_{2} \mathrm{O}$

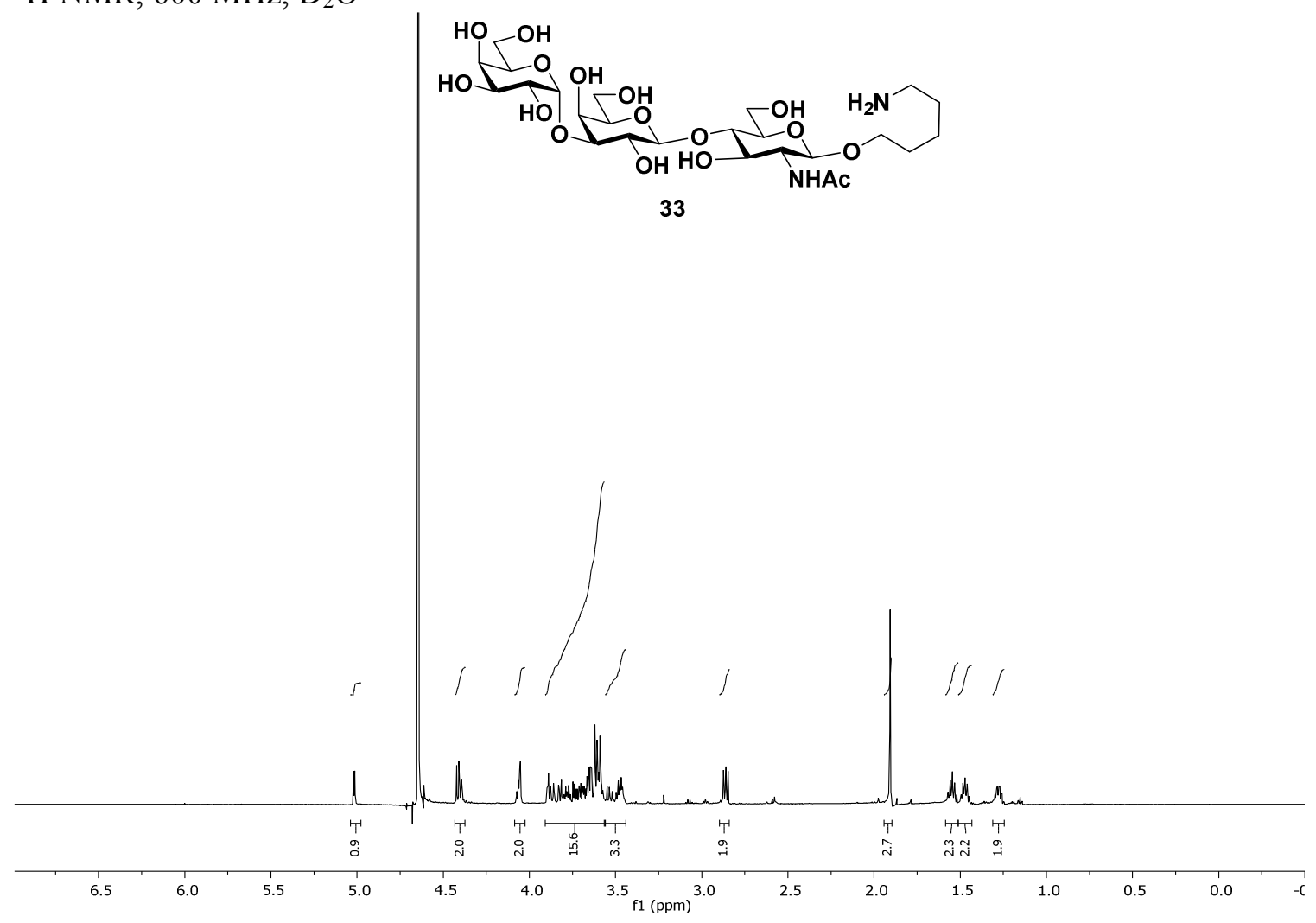

${ }^{13} \mathrm{C}$ NMR, $150 \mathrm{MHz}, \mathrm{D}_{2} \mathrm{O}$
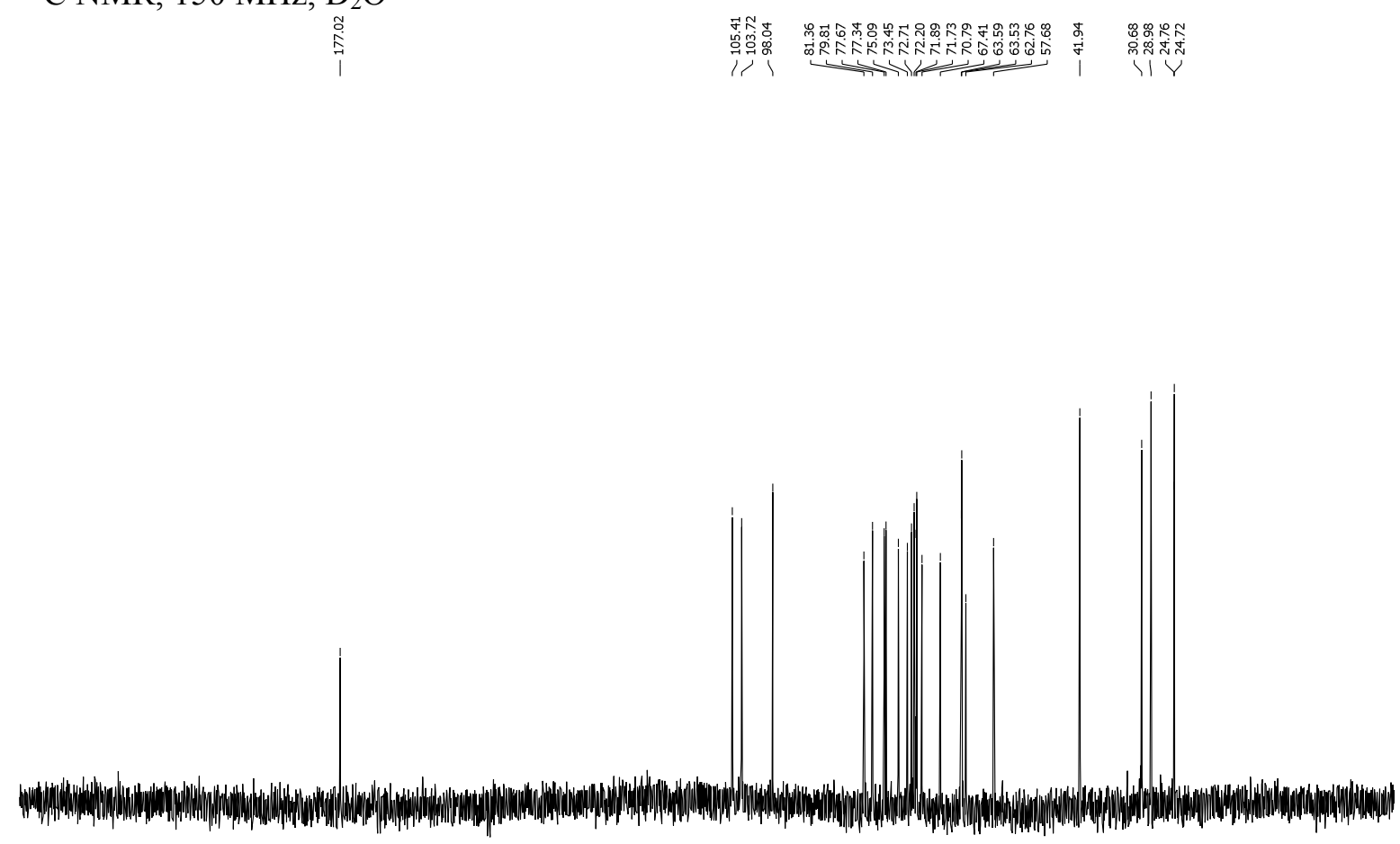

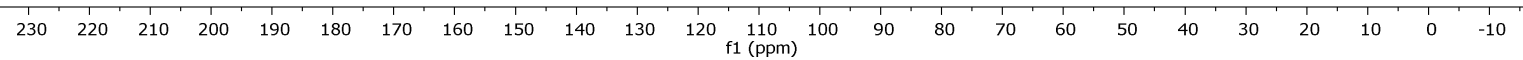

